Métodos algébricos para a
obtenção de formas gerais
reversíveis-equivariantes

Iris de Oliveira 


\title{
Métodos algébricos para a obtenção de formas gerais reversíveis-equivariantes
}

\author{
Iris de Oliveira \\ Orientadora: Profa. Dra. Miriam Garcia Manoel
}

Dissertação apresentada ao Instituto de Ciências Matemáticas e de Computação - ICMC-USP, como parte dos requisitos para obtenção do título de Mestre em Matemática.

USP - São Carlos

Março/2009 
- Mos meus pais III 



\section{Agradecimentos}

Primeiramente agradeço a Deus, pois sem Ele nenhum dos meus sonhos se tornaria realidade.

Obrigada, Pai e Mãe, por me darem a oportunidade de estudo, me dar forças e encorajamento em todos os momentos da minha vida, por todas as incessantes orações e por me amar incondicionalmete. Obrigada, Carol, Gabi, Danda, Iara, Jefim e Samuel. Apesar da distância e da eterna saudade vocês foram o meu porto seguro por todo esse tempo!

Agradeço ao Rodrigo pelo amor e paciência para comigo e por me fazer acreditar em

mim. À sua mãe, Verônica, pelas palavras mansas de conforto, e por sempre me receber bem em sua casa.

À professora Miriam pelo tema proposto, pela seriedade e participação efetiva na realização desse trabalho. Obrigada pelo encorajamento e motivação para prosseguir com o mestrado.

Aos professores Alan, Liane e Jamil, por todo o apoio que me deram durante a minha graduação e incentivo para cursar o mestrado.

À todos os meus amigos da minha cidade natal, Nova Venécia, pelo incentivo a vir para São Carlos estudar.

À todos os amigos que encontrei nesse Instituto, aos meus colegas do mestrado pelos momentos de estudo e descontração, em especial a Jaqueline pela sincera amizade e pelos momentos de desabafos. Foram tantos!

À todos os professores e funcionários desse Instituto que fizeram parte dessa conquista.

À CAPES pelo suporte finaceiro concedido durante parte do mestrado.

Enfim, à todos aqueles que colaboraram de alguma forma para a realização desse trabalho. 



\section{Resumo}

Na análise global e local de sistemas dinâmicos assumimos, em geral, que as equações estão numa forma normal. Em presença de simetrias, as equações e o domínio do problema são invariantes pelo grupo formado por estas simetrias; neste caso, o campo de vetores é equivariante pela ação deste grupo. Quando, além das simetrias, temos também ocorrência de anti-simetrias - ou reversibilidades - as equações e o domínio do problema são ainda invariantes pelo grupo formado pelo conjunto de todas as simetrias e anti-simetrias; neste caso, o campo de vetores é reversível-equivariante. Existem muitos modelos físicos onde simetrias e anti-simetrias aparecem naturalmente e cujo efeito pode ser estudado de uma forma sistemática através de teoria de representação de grupos de Lie. O primeiro passo deste processo é colocar a aplicação que modela tal sistema numa forma normal e isto é feito com a dedução a priori da forma geral dos campos de vetores. Esta forma geral depende de dois componentes: da base de Hilbert do anel das funções invariantes e dos geradores do módulo das aplicações reversíveis-equivariantes. Neste projeto, nos concentramos principalmente na aplicação de resultados recentes da literatura para a construção de uma lista de formas gerais de aplicações reversíveisequivariantes sob a ação de diferentes grupos. Além disso, adaptamos ferramentas algébricas da literatura existentes no contexto equivariante para o estudo sistemático de acoplamento de células idênticas no contexto reversível-equivariante. 



\section{Abstract}

In the global and local analysis of dynamical systems, we assume, in general, that the equations are in a normal form. In presence of symmetries, the equations and the problem domain are invariant under the group formed by these symmetries; in that case, the vector field is equivariant by the action of this group. When, in addition to the symmetries, we have the occurrence of anti-symmetries - or reversibility - the equations and the problem domain are still invariant by the group formed by the set of all symmetries and anti-symmetries; in this case, the vector field is reversible-equivariant. There are many physical models where both symmetries and anti-symmetries occur naturally and whose effect can be studied in a systematic way through group representation theory. The first step of this process is to put the mapping that model the system in a normal form, and this is done with the deduction of the general form of the vector field. This general form depends on two components: the Hilbert basis of the invariant function ring and also the generators of the module of the revesible-equivariants. In this work, we mainly focus on the applications of recent results of the literature to build a list of general forms of reversible-equivariant mappings under the action of different groups. We also adapt algebraic tools of the existing literature in the equivariant context to the systematic study of coupling of identical cells in the reversible-equivariant context. 



\section{Sumário}

Notações $\quad$ xii

Introdução $\quad 1$

1 Preliminares 5

1.1 Teoria de grupos . . . . . . . . . . . . . . . . 5

1.1.1 Grupos de Lie . . . . . . . . . . . . . . . . . . 5

1.1 .2 Ação e representação . . . . . . . . . . . . . . . . . . . . . . 7

1.1.3 Integral de Haar . . . . . . . . . . . . . . . . . . . . . . 9 9

1.2 Irredutibilidade . . . . . . . . . . . . . . . . . . . . . . . . 10

1.3 Germes de funções invariantes e de aplicações

equivariantes . . . . . . . . . . . . . . . . . . . 14

1.3.1 Germes de funções invariantes . . . . . . . . . . . . . . . . . . 15

1.3.2 Germes de aplicações equivariantes . . . . . . . . . . . . . . . . 18

1.4 Órbitas e subgrupos de isotropia . . . . . . . . . . . . . . . 25

1.4.1 Órbitas, isotropias e subespaço de ponto fixo . . . . . . . . . 25

1.4.2 Subespaço de ponto fixo e a fórmula do traço . . . . . . . . . 28

2 A teoria reversível-equivariante $\quad 33$

2.1 O contexto reversível-equivariante . . . . . . . . . . . . . . 34

2.2 Teoria de caracteres . . . . . . . . . . . . . . . . . . . . . . . . . . . . . . . . . . . 35

2.2.1 Caracter de uma representação . . . . . . . . . . . . . . . . . . . 35

2.2.2 Séries de Hilbert-Poincaré e fórmulas de Molien . . . . . . . . . . 37

2.2.3 Fórmulas de caracter . . . . . . . . . . . . . . . . . . . . 40

2.3 Algoritmos . . . . . . . . . . . . . . . . . . . . 43

2.3.1 Operadores de Reynolds . . . . . . . . . . . . . . . . . . . 43

2.3.2 Obtenção de formas gerais anti-invariantes . . . . . . . . . . . . . 49

2.3.3 Obtenção de formas gerais reversíveis-equivariantes . . . . . . . . 53

2.4 O Pêndulo simples . . . . . . . . . . . . . . . . . . . . . . . . 54

3 Produto Coroa $\quad 57$

3.1 O produto coroa $\Gamma$ 々 $\mathbf{S}_{N}$ como grupo de simetrias . . . . . . . $\quad 57$

3.1.1 Geradores dos equivariantes para o grupo $\mathbf{O}(2)<\mathbf{S}_{N} \ldots \ldots . . .58$

3.2 O produto coroa $\Gamma$ ? $\mathbf{S}_{N}$ como grupo de simetrias e anti-simetrias . . . . 62

3.2.1 Geradores dos reversíveis-equivariantes para o grupo

$\mathbf{O}(2) \imath \mathbf{S}_{N} \ldots \ldots \ldots \ldots \ldots \ldots$ 
$\begin{array}{lll}4 & \text { Exemplos } & 67\end{array}$

4.1 Forma geral $\mathbf{Z}_{2}$-reversível-equivariante . . . . . . . . . . . . . . 67

4.2 Forma geral $\mathbf{Z}_{4}$-reversível-equivariante . . . . . . . . . . . . . . . 68

4.3 Forma geral $\mathbf{Z}_{2} \oplus \mathbf{Z}_{2}$-reversível-equivariante . . . . . . . . . . . . . . 70

4.4 Forma geral $\mathbf{O}(2)$-reversível-equivariante . . . . . . . . . . . . . . 71

4.5 Forma geral $\mathbf{D}_{n}$-reversível-equivariante . . . . . . . . . . . . . . . . . 72

4.5.1 Forma geral $\mathbf{D}_{4}(\kappa)$-reversível-equivariante . . . . . . . . . 75

4.5.2 Forma geral $\mathbf{D}_{4}(R)$-reversível-equivariante . . . . . . . . . . . . 75

4.6 Forma geral $\left(\mathbf{D}_{6}+\mathbf{T}^{2}\right) \oplus \mathbf{Z}_{2}$-reversível-equivariante . . . . . . . . . . 76

4.7 Forma geral $\mathbb{O}$-reversível-equivariante . . . . . . . . . . . . . . . 78

4.8 Forma geral $\mathbf{S}_{n}$-reversível-equivariante . . . . . . . . . . . . . . . . 79

4.8.1 Forma geral $\mathbf{S}_{2}$-reversível-equivariante . . . . . . . . . . . 80

4.8.2 Forma geral $\mathbf{S}_{3}$-reversível-equivariante . . . . . . . . . . . . . 81

$\begin{array}{lr}\text { Bibliografia } & 83\end{array}$

$\begin{array}{lr}\text { Índice remissivo } & 84\end{array}$ 


\section{Lista de Figuras}

1.1 Órbitas da ação do $\mathbf{D}_{5}$ em $\mathbb{C} . \quad \ldots \ldots \ldots$. . . . . . . . . . . . 27

2.1 Retratos de fase de campos vetoriais reais com simetrias e anti-simetrias. 33

2.2 O pêndulo. . . . . . . . . . . . . . . . . . 54

2.3 Retrato de fase do pêndulo sem atrito . . . . . . . . . . . . . . 55

4.1 Campo vetorial $\mathbf{Z}_{4}$-reversível-equivariante dado em 4.1. . . . . . . . . . . 70

4.2 Campo vetorial $\mathbf{Z}_{4}$-reversível-equivariante dado em $4.2 \ldots \ldots$. . . . . . 70

4.3 Campo vetorial $\mathbf{D}_{6}$-reversível-equivariante dado em $4.3 \ldots \ldots$. . . . . . . . 74

4.4 Campo vetorial $\mathbf{D}_{4}$-reversível-equivariante dado em $4.4 \ldots$. . . . . . . . . 74 



\section{Notações}

$\Gamma$ : Grupo de Lie compacto agindo em um espaço vetorial $V$ de dimensão finita;

$\Gamma_{+}$: Subgrupo de $\Gamma$ de índice 2, formado pelas simetrias de $\Gamma$;

$\Gamma_{-}$: Subconjunto formado pelas anti-simetrias de $\Gamma$;

$\sigma:$ Homomorfismo de $\Gamma$ em $\mathbf{Z}_{2}$;

$(\rho, V)$ : Espaço vetorial $V$ sob a representação $\rho$ de $\Gamma$;

$\rho_{\sigma}:$ Representação $\sigma$-dual de $\rho$;

$\int_{\Gamma}$ : Integral de Haar normalizada sobre $\Gamma$;

$\Sigma_{x}$ : Subgrupo de isotropia de $x \in V$;

$\Gamma_{x}$ : Órbita da ação de $\Gamma$ em $x \in V$;

$\operatorname{Fix}_{V}(\Sigma)$ : Subespaço de ponto fixo de um subgrupo $\Sigma \subset \Gamma$ em $(\rho, V)$;

$\mathcal{P}_{V}(\Gamma)$ : Anel das funções polinomiais $f: V \rightarrow \mathbb{R} \Gamma$-invariantes;

$\mathcal{E}_{V}(\Gamma):$ Anel dos germes de funções $f:(V, 0) \rightarrow \mathbb{R} \Gamma$-invariantes;

$\mathcal{Q}_{V}(\Gamma)$ : Módulo sobre $\mathcal{P}_{V}(\Gamma)$ das funções polinomiais $f: V \rightarrow \mathbb{R} \Gamma$-anti-invariantes;

$\mathcal{F}_{V}(\Gamma)$ : Módulo sobre $\mathcal{E}_{V}(\Gamma)$ dos germes de funções $f:(V, 0) \rightarrow \mathbb{R} \Gamma$-anti-invariantes;

$\overrightarrow{\mathcal{P}}_{V, W}(\Gamma)$ : Módulo sobre $\mathcal{P}_{V}(\Gamma)$ das aplicações polinomiais $g: V \rightarrow W \Gamma$-equivariantes;

$\overrightarrow{\mathcal{E}}_{V, W}(\Gamma)$ : Módulo sobre $\mathcal{E}_{V}(\Gamma)$ dos germes de aplicações $g:(V, 0) \rightarrow W$ r-equivariantes;

$\overrightarrow{\mathcal{Q}}_{V, W}(\Gamma)$ : Módulo sobre $\mathcal{P}_{V}(\Gamma)$ das aplicações polinomiais $g: V \rightarrow W \Gamma$-reversíveisequivariantes;

$\overrightarrow{\mathcal{F}}_{V, W}(\Gamma)$ : Módulo sobre $\mathcal{E}_{V}(\Gamma)$ dos germes de aplicações $g:(V, 0) \rightarrow W \Gamma$-reversíveisequivariantes;

$\mathcal{X}_{V}:$ Caracter correspondente à representação de $\Gamma$ em $V$;

$V \otimes W:$ Produto tensorial entre os espaços $V$ e $W$; 
$V^{\otimes n}:$ Produto tensorial $\underbrace{V \otimes \ldots \otimes V}_{n \text { vezes }}$;

$V \oplus W$ : Soma direta entre os espaços $V$ e $W$;

$S^{n} V$ : A $n$-ésima potência tensorial simétrica;

$\mathcal{P}_{V}$ : Anel das funções polinomiais $V \rightarrow \mathbb{R}$

$\mathcal{P}_{V}^{d}:$ Espaço vetorial dos polinômios homogêneos $V \rightarrow \mathbb{R}$ de grau $d$;

$\mathcal{P}_{V}^{d}(\Gamma)$ : Espaço vetorial dos polinômios homogêneos $\Gamma$-invariantes $V \rightarrow \mathbb{R}$ de grau $d$;

$\overrightarrow{\mathcal{P}}_{V, W}:$ Espaço vetorial das aplicações polinomiais $V \rightarrow W$

$\overrightarrow{\mathcal{P}}_{V, W}^{d}:$ Espaço vetorial das aplicações polinomiais homogêneas $V \rightarrow W$ de grau $d$;

$\overrightarrow{\mathcal{P}}_{V, W}^{d}(\Gamma)$ : Espaço vetorial das aplicações polinomiais homogêneas $V \rightarrow W$ de grau $d$ $\Gamma$-equivariantes;

$\mathcal{Q}_{V}^{d}$ : Espaço vetorial dos polinômios homogêneas $V \rightarrow \mathbb{R}$ de grau $d \Gamma$-anti-invariantes;

$\overrightarrow{\mathcal{Q}}_{V, W}^{d}$ : Espaço vetorial das aplicações polinomiais homogêneas $V \rightarrow W$ de grau $d \Gamma$ reversíveis-equivariantes;

$\Phi_{V}^{\Gamma}(t)$ : Série de Hilbert para o anel $\mathcal{P}_{V}(\Gamma)$

$\Psi_{V, W}^{\Gamma}(t)$ : Série de Hilbert para o anel $\overrightarrow{\mathcal{P}}_{V, W}(\Gamma)$;

$\Gamma$ 乙 $\mathbf{S}_{N}$ : Produto coroa entre os grupos $\Gamma$ e $\mathbf{S}_{N}$;

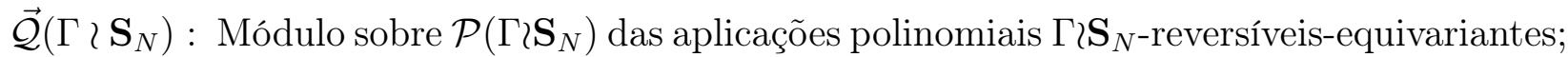
$\overrightarrow{\mathcal{Q}}\left(\Gamma^{N}\right)$ : Módulo sobre $\mathcal{P}\left(\Gamma^{N}\right)$ das aplicações polinomiais $\Gamma^{N}$-reversíveis-equivariantes. 


\section{Introdução}

De maneira geral, um sistema dinâmico é um sistema que é investigado com o passar do tempo, seja ele natural, físico, químico, dentre outros. Dizemos que é sistema dinâmico contínuo ou discreto, dependendo se a variação do tempo é considerada contínua ou discreta, respectivamente. As soluções variando com o tempo descritas geometricamente no espaço de configurações são chamadas trajetórias. O modelo matemático proposto para o estudo de tais sistemas depende essencialmente de uma aplicação definida no espaço de configurações. A descrição formal das características observadas no sistema reflete as restrições a serem impostas em tal aplicação. Uma característica que entra de forma natural em muitos modelos é a ocorrência de simetrias e anti-simetrias. O conjunto $\Gamma$ de tais elementos tem estrutura de grupo, que para nossos propósitos supomos ser um grupo de Lie compacto. Sistemas dinâmicos sob a ação dos elementos de $\Gamma$ são chamados sistemas $\Gamma$-reversíveis-equivariantes. Notamos aqui que o subconjunto $\Gamma_{+}$das simetrias é um subgrupo normal de índice 2 em $\Gamma$, o que é uma propriedade fundamental para a obtenção dos resultados que apresentamos neste trabalho.

Do ponto de vista da dinâmica, simetrias levam trajetórias em trajetórias preservando a orientação com a variação do tempo; as anti-simetrias, ou também chamadas reversibilidades, levam trajetórias em trajetórias, mas revertendo a orientação segundo a variação do tempo.

Nos últimos dez anos aproximadamente tem havido grande interesse pelo estudo dos sistemas dinâmicos reversíveis-equivariantes, como em [3], [4], [5], [7], [8], [17], [18] e outros. Nossa abordagem para o estudo sistemático neste contexto segue principalmente [4] e se dá através da teoria de ação e representação de grupos no espaço de configurações, que em nosso caso, por sua vez, é um espaço vetorial $V$ de dimensão finita.

As aplicações que regem sistemas dinâmicos $\Gamma$-reversíveis-equivariantes têm uma forma geral bem determinada uma vez conhecido o grupo $\Gamma$. Para se construir esta forma geral, é necessário se conhecer a teoria invariante para aquela particular ação (ou representação). O estudo começa considerando-se um homomorfismo de grupos

$$
\sigma: \Gamma \rightarrow \mathbf{Z}_{2}
$$

onde $\mathbf{Z}_{2}$ é o grupo multiplicativo $\{-1,1\}$ e $\Gamma_{+}=\operatorname{ker} \sigma$. Assim, um elemento $\gamma \in \Gamma$ é chamado de simetria se $\sigma(\gamma)=1$ e é chamado de anti-simetria, ou simetria reversível, se $\sigma(\gamma)=-1$. Denotamos por $\Gamma_{-}$o conjunto de todas as anti-simetrias do grupo $\Gamma$.

Dizemos que uma aplicação $g: V \rightarrow V$ - ou um germe, quando for o caso - é $\Gamma$-reversível-equivariante se para todo $\gamma \in \Gamma$ e $x \in V$ temos

$$
g(\gamma x)=\sigma(\gamma) \gamma g(x) .
$$


Se $\Gamma_{-}$é vazio, $g$ é chamada de (puramente) $\Gamma$-equivariante.

As ferramentas que estudamos neste trabalho têm o propósito de se calcular a forma geral de aplicações $\Gamma$-reversíveis-equivariantes a partir da teoria $\Gamma_{+}$-invariante. Para tanto, apresentamos resultados em sistemas $\Gamma$-reversíveis-equivariantes que podem ser obtidos através do conhecimento de resultados correspondentes de sistemas puramente equivariantes, a saber $\Gamma$-equivariantes e $\Gamma_{+}$-equivariantes.

Em nosso trabalho, vamos ilustrar a ocorrência de simetrias e anti-simetrias nos sistemas dinâmicos contínuos regidos por um sistema de EDOs

$$
\dot{x}=g(x),
$$

com $g$ satisfazendo (0.2). Neste caso, a equação (0.3) é invariante pela ação do grupo $\Gamma$ pela transformação $(x, t) \mapsto(\gamma x, \sigma(\gamma) t)$. Assim a construção do homomorfismo $\sigma$ como em (0.1) reflete a característica da ação das simetrias de $\Gamma$ preservar a direção das trajetórias no tempo e a ação das anti-simetrias reverter a direção das trajetórias no tempo.

Descrevemos a seguir o conteúdo dos capítulos desta dissertação. As referências centrais que guiaram os estudos são [15], para o contexto equivariante e [3] e[4] para a teoria e resultados recentes no contexto reversível-equivariante.

No Capítulo 1, introduzimos os conceitos básicos sobre grupos de Lie compactos, juntamente com a teoria invariante e equivariante.

No Capítulo 2, apresentamos conceitos, definições e resultados para o contexto reversível-equivariante. Primeiramente, combinamos a teoria de invariantes com a teoria de caracter para obter resultados que fornecem uma fórmula para a dimensão do espaço vetorial das aplicações polinomiais reversíveis-equivariantes de cada grau. O conhecimento destas dimensões pode ser um dado útil para a dedução das formas gerais, em especial nos casos em que a aplicação dos algoritmos que mencionamos a seguir se torna complicada, por exemplo por razões numéricas. A seguir, tratamos então dos algoritmos de obtenção das formas gerais reversíveis-equivariantes, que é nosso tema principal: o primeiro passo é usar a demonstração do Teorema de Poénaru (Teorema 1.3.18) que nos fornece um método para se calcular geradores do módulo das aplicações $\Gamma_{+}$-equivariantes sobre $V$ a partir do conhecimento de uma base de Hilbert para o anel das funções $V \times W \rightarrow \mathbb{R} \Gamma_{+}$-invariantes. Em muitos exemplos, esta base de Hilbert pode ser obtida diretamente com o uso do programa Singular [21]. Feito este passo, veremos que podemos aplicar diretamente, e sem dificuldades adicionais, os algoritmos apresentados na Seção 2.3 para obtermos os geradores do módulo das aplicações reversíveis-equivariantes.

No Capítulo 3, consideramos o problema de acoplamento de células idênticas, cada uma representando um sistema reversível-equivariante pela ação de um grupo de Lie compacto $\Gamma$. No caso de $N$ células, o grupo de simetrias e anti-simetrias é dado pelo produto coroa $\Gamma\left\langle\mathbf{S}_{N}\left(=\Gamma^{N} \dot{+} \mathbf{S}_{N}\right)\right.$, onde $\mathbf{S}_{N}$ é o grupo das permutações de $N$ elementos. $\mathrm{O}$ acoplamento de células puramente equivariantes foi tratado na literatura primeiramente por Golubitsky et al [10] e depois disso por muitos outros autores. Nesta dissertação apresentamos o formalismo para o caso em que cada célula é reversível equivariante. Apresentamos primeiramente a ação apropriada do grupo $\Gamma$ < $\mathbf{S}_{N}$ no espaço $V^{N}$ das configurações. Em seguida, aplicamos a teoria para a obtenção da forma geral das aplicações $\Gamma$-reversíveis-equivariantes para $\Gamma=\mathbf{O}(2)$ 乙 $\mathbf{S}_{N}$. 
No Capítulo 4 apresentamos uma série de exemplos de construção de aplicações reversíveis-equivariantes, onde usamos o programa Singular num primeiro passo e os algoritmos num segundo passo. Os grupos escolhidos nestes exemplos aparecem frequentemente na literatura, de forma que esperamos que esta possa ser uma contribuição desta dissertação a quem trabalhe nesta linha de pesquisa. Além disso, apresentamos os cálculos e os resultados de forma sistemática, organizada em cinco etapas em cada exemplo, com o objetivo de facilitar a quem interesse o uso do procedimento para a contrução de formas gerais de aplicações reversíveis-equivariantes pela ação de outros grupos que não os apresentamos aqui. 



\section{Capítulo 1}

\section{Preliminares}

Começamos a teoria com o estudo de um grupo de Lie compacto $\Gamma$, agindo linearmente num espaço vetorial real $V$ de dimensão finita, e descrevemos exemplos fundamentais incluindo o grupo ortogonal $\mathbf{O}(n)$, o grupo do círculo $\mathbf{S}^{1}$, o grupo diedral $\mathbf{D}_{n} \mathrm{e}$ o n-toro $\mathbf{T}^{n}$.

Um determinado grupo $\Gamma$ pode agir em um espaço de várias formas e por isso introduzimos a noção de representação e ação de grupos. Vamos ver que a teoria de representação de um grupo de Lie compacto tem estruturas especiais, por exemplo, a existência da integral de Haar invariante pela ação deste grupo. Isto nos permite assumir que um grupo de Lie compacto $\Gamma$ age por transformações ortogonais sobre $V$.

Descrevemos a decomposição de uma representação em uma soma direta de representações mais simples, as quais são invariantes por $\Gamma$, sendo o processo de decomposição do espaço $V$ semelhante ao processo de diagonalização de matrizes.

Definimos as aplicações equivariantes e enunciamos os teoremas principais dessa parte: o Teorema de Hilbet-Weyl, que garante a existência de uma base finita para o anel das funções polinomiais invariantes, e o Teorema de Schwarz, que afirma que essa base também gera os germes de funções invariantes.

Apresentamos ainda o subespaço de ponto fixo de um subgrupo $\Sigma$ de $\Gamma$, a órbita de um elemento de $V$ e o subgrupo de isotropia de um elemento do espaço $V$. Em seguida, apresentamos um método, conhecido como fórmula do traço, para o cálculo da dimensão do subespaço de ponto fixo.

\subsection{Teoria de grupos}

Seja GL $(n)$ o grupo das matrizes invertíveis de ordem $n$. Notemos que GL $(n)$ é um subgrupo aberto do grupo das matrizes de ordem $n \mathbb{M}_{n \times n}(\mathbb{R})$, uma vez que é o complementar da imagem inversa de $\{0\}$ pela aplicação determinande det: $\mathbb{M}_{n \times n}(\mathbb{R}) \rightarrow$ $\mathbb{R}$.

\subsubsection{Grupos de Lie}

Para nossa finalidade definimos:

Definição 1.1.1. Um grupo de Lie linear é um subgrupo $\Gamma$ fechado de $\boldsymbol{G L}(n)$. 
Se o grupo $\Gamma$ for compacto, o chamamos apenas Grupo de Lie, pois todo grupo de Lie compacto é topologicamente isomorfo a um grupo de Lie linear (Veja Golubitsky [15]).

Geralmente nos referimos a certos grupos como sendo grupos de Lie, porém são apenas subgrupos isomorfos a algum subgrupo fechado do GL $(n)$. Por exemplo, nos referimos ao grupo multiplicativo $\mathbf{Z}_{2}=\{-1,1\}$ como grupo de Lie, já que existe um isomorfismo com o grupo $\left\{-I_{n}, I_{n}\right\} \subset \mathbf{G L}(n)$, onde $I_{n}$ é a matriz identidade $n \times n$.

Apresentamos alguns exemplos de grupos de Lie:

Exemplos 1.1.2. $\quad$ 1. O grupo ortogonal $\mathbf{O}(n)$, formado por todas as matrizes $A \subset$ $\mathbb{M}_{n \times n}(\mathbb{R})$ tal que

$$
A A^{t}=I_{n}
$$

onde $A^{t}$ denota a matriz transposta de $\mathrm{A}$.

2. O grupo especial ortogonal $\mathbf{S O}(n)$ formado por todas as matrizes $A \subset \mathbf{O}(n)$ tal que $\operatorname{det} A=1$. Esse grupo também é chamado de grupo de rotações em $\mathbb{R}^{n}$. Em particular, $\mathbf{S O}(2)$ consiste das rotações no plano

$$
R_{\theta}=\left(\begin{array}{cc}
\cos \theta & -\operatorname{sen} \theta \\
\operatorname{sen} \theta & \cos \theta
\end{array}\right), \theta \in[0,2 \pi]
$$

Podemos identificar $\mathbf{S O}(2)$ com o grupo $\mathbf{S}^{1}$ através da aplicação:

$$
R_{\theta} \mapsto e^{i \theta}, \theta \in[0,2 \pi]
$$

O grupo $\mathbf{O}(2)$ é gerado por $\mathbf{S O}(2)$ juntamente com a reflexão

$$
\kappa=\left(\begin{array}{cc}
1 & 0 \\
0 & -1
\end{array}\right)
$$

3. Seja $\mathbf{Z}_{n}$ o grupo cíclico de ordem $n$. Podemos identificar $\mathbf{Z}_{n}$ com o grupo das matrizes $2 \times 2$ geradas pela rotação de ângulo $\xi=\frac{2 \pi}{n}$, isto é, por $R_{\xi}$.

4. O grupo diedral $\mathbf{D}_{n}$ com ordem $2 n$ é gerado pelo grupo $\mathbf{Z}_{n}$ e um elemento de ordem 2 que não comuta com $\mathbf{Z}_{n}$. Geometricamente $\mathbf{D}_{n}$ é o grupo de simetrias do polígono regular de $n$ lados. Indicamos por $\mathbf{D}_{n}$ o grupo de matrizes geradas pela rotação $R_{\frac{2 \pi}{n}}$ e pela reflexão $\kappa$ definida em (1.1).

5. O Toro $n$-dimensional, $\mathbf{T}^{n}=\underbrace{\mathbf{S}^{1} \times \ldots \times \mathbf{S}^{1}}_{n \text { vezes }}$, isomorfo a um grupo de Lie pela aplicação que leva $\theta=\left(\theta_{1}, \ldots, \theta_{n}\right) \in \mathbf{T}_{n}$ à matriz

$$
\left(\begin{array}{cccc}
R_{\theta_{1}} & 0 & \ldots & 0 \\
0 & R_{\theta_{2}} & \cdots & 0 \\
\vdots & \vdots & \ddots & \vdots \\
0 & 0 & 0 & R_{\theta_{n}}
\end{array}\right) \in \mathbf{G L}(2 n)
$$


6. $\mathbb{R}^{n}$ é isomorfo ao grupo de Lie das matrizes da forma

$$
\left(\begin{array}{ccccc}
1 & a_{1} & a_{2} & \ldots & a_{n} \\
0 & 1 & 0 & \ldots & 0 \\
0 & 0 & 1 & \ldots & 0 \\
\vdots & \vdots & \vdots & \ddots & \vdots \\
0 & 0 & 0 & \ldots & 1
\end{array}\right) \in \mathbf{G L}(n+1)
$$

$\operatorname{com} a_{j} \in \mathbb{R}$ para $i=1, \ldots, n$.

7. Todo grupo finito é isomorfo a um grupo de Lie.

Notemos que $\mathbf{O}(n), \mathbf{S O}(n), \mathbf{T}^{n}$ e todos o grupos finitos são grupos de Lie compactos.

Como um subconjunto do $\mathbb{M}_{n \times n}(\mathbb{R})$ o grupo $\Gamma$ se fatora em componentes conexas, observando que se $\Gamma$ é um grupo de Lie compacto então $\Gamma$ tem um número finito de componentes conexas. A componente conexa que contém a identidade é denotada por $\Gamma^{0}$. Por exemplo, $\mathbf{O}(n)^{0}=\mathbf{S O}(n)$. Sendo uma componente conexa, $\Gamma^{0}$ é fechado em $\Gamma$. Daí, $\Gamma^{0}$ é fechado em GL $(n)$ se $\Gamma$ também for. Portanto, $\Gamma^{0}$ é um grupo de Lie e é compacto se $\Gamma$ também for compacto.

\subsubsection{Ação e representação}

As simetrias de um sistema dinâmico regido por equações diferenciais ordinárias ou parciais são especificadas em termos de um grupo de tranformações que preservam a estrutura da equação. Este grupo é conhecido como grupo das simetrias do sistema e em nosso estudo é essencial descrever sua estrutura e sua ação no espaço das variáveis.

Definição 1.1.3. Seja $\Gamma$ um grupo de Lie e $V$ um espaço vetorial de dimensão finita. Dizemos que $\Gamma$ age linearmente (ou simplesmente age) em $V$ se existe uma aplicação continua $\Gamma \times V \rightarrow V,(\gamma, v) \mapsto \gamma \cdot v$, tal que:

a) Para cada $\gamma \in \Gamma$, a aplicação $\rho_{\gamma}: V \rightarrow V$ definida por $\rho_{\gamma} v=\gamma \cdot v$ é linear e invertível;

b) Se $\gamma_{1}, \gamma_{2} \in \Gamma$, então $\gamma_{1} \cdot\left(\gamma_{2} \cdot v\right)=\left(\gamma_{1} \gamma_{2}\right) \cdot v$ para todo $v \in V$.

Definição 1.1.4. A uma ação de $\Gamma$ em $V$ corresponde um homomorfismo de grupos

$$
\begin{aligned}
\rho: \Gamma & \rightarrow \boldsymbol{G L}(V) \\
\gamma & \mapsto \rho_{\gamma}
\end{aligned}
$$

chamado representação de $\Gamma$ em $V$, onde $\boldsymbol{G L}(V)$ denota o espaço das aplicações lineares invertiveis $V \rightarrow V$.

Toda ação induz uma representação. Enquanto uma ação mostra como um elemento $\gamma \in \Gamma$ transforma um dado elemento $v \in V$, uma representação mostra como $\gamma$ transforma todo o espaço $V$. Uma ação de um grupo $\Gamma$ pode ser definida especificando apenas a ação para os geradores do grupo, desde que ela seja linear. Vamos denotar por $(\rho, V)$ o espaço $V$ sob a representação $\rho$ de $\Gamma$. Quando não houver ambiguidade sobre a ação, usaremos apenas $\gamma v$ no lugar de $\gamma \cdot v$. 
Exemplos 1.1.5. 1. Todo grupo de Lie $\Gamma$ é um grupo de matrizes em GL $(n)$ para algum $n$. E uma ação natural, denomidada ação padrão, é dada por

$$
\begin{aligned}
\Gamma \times V & \rightarrow V \\
(A, v) & \mapsto A \cdot v=A v .
\end{aligned}
$$

2. Todo grupo de Lie $\Gamma$ tem uma ação trivial em $V$ :

$$
\begin{aligned}
\Gamma \times V & \rightarrow V \\
(\gamma, v) & \mapsto \gamma \cdot v=v .
\end{aligned}
$$

3. Para cada inteiro $k$, o grupo $\mathbf{S}^{1}$ tem uma ação natural em $\mathbb{C}$ definida por

$$
\begin{aligned}
\mathbf{S}^{1} \times \mathbb{C} & \rightarrow \mathbb{C} \\
(\theta, z) & \mapsto \theta \cdot z=e^{i k \theta} z .
\end{aligned}
$$

Se $k=0$ temos a ação trivial. A ação para $k=1$ corresponde à representação de $\mathbf{S}^{1}$ para o qual $\rho_{\theta}$ é uma matriz de rotação de ângulo $\theta$ em $\mathbb{R}^{2}$ :

$$
R_{\theta}=\left(\begin{array}{cc}
\cos \theta & -\operatorname{sen} \theta \\
\operatorname{sen} \theta & \cos \theta
\end{array}\right)
$$

Neste caso, existe uma ação natural de $\mathbf{S O}(2)$ em $\mathbb{R}^{2}$, dada pela multiplicação de matrizes definida em (1.2).

4. Cada ação de $\mathbf{S}^{1} \equiv \mathbf{S O}(2)$ definida em (1.4) induz uma ação de $\mathbf{O}(2)$ em $\mathbb{C}$ se considerarmos

$$
\begin{aligned}
& \theta \cdot z=e^{i k \theta} z \\
& \kappa \cdot z=\bar{z} .
\end{aligned}
$$

Note que $\mathbf{O}(2)=\langle\mathbf{S O}(2), \kappa\rangle$.

5. Todo grupo de Lie $\Gamma \subset \mathbf{G L}(n)$ age no espaço das matrizes $\mathbb{M}_{n \times n}(\mathbb{R})$ como

$$
\gamma \cdot A=\gamma A \gamma^{-1}
$$

6. Como o grupo diedral $\mathbf{D}_{n}$ é gerado pela rotação de ângulo $\xi=\frac{2 \pi}{n}$ e pela reflexão $\kappa$, então existe uma ação de $\mathbf{D}_{n}$ no $\mathbb{R}^{2}$ dada pela ação padrão, isto é,

$$
R_{\theta} \cdot(x, y)=\left(\begin{array}{cc}
\cos \xi & -\operatorname{sen} \xi \\
\operatorname{sen} \xi & \cos \xi
\end{array}\right)\left(\begin{array}{l}
x \\
y
\end{array}\right), \kappa \cdot(x, y)=\left(\begin{array}{cc}
1 & 0 \\
0 & -1
\end{array}\right)\left(\begin{array}{l}
x \\
y
\end{array}\right)
$$

Em particular, existe uma ação do $\mathbf{D}_{n}$ em $\mathbb{C}$ caracterizada como em (1.6).

Duas ações podem ser isomorfas no seguinte contexto: 
Definição 1.1.6. Sejam $(\rho, V)$ e $(\eta, W)$ representações de $\Gamma$. Dizemos que as representações $\rho$ e $\eta$ (ou os espaços $V$ e W) são $\Gamma$-isomorfas se existir um isomorfismo linear $A: V \rightarrow W$ tal que

$$
A\left(\rho_{\gamma} v\right)=\eta_{\gamma} A(v)
$$

Sem perda de generalidade podemos estender essa idéia para o caso em que $\Gamma$ age em $V$ e um grupo $\Delta$, isomorfo a $\Gamma$, age em $W$. Por exemplo, as ações de $\mathbf{O}(2)$ para os inteiros $k$ e $-k$ definidas em (1.4) são isomorfas. De fato, basta tomar o isomorfismo linear $A: \mathbb{C} \rightarrow \mathbb{C}, A(z)=\bar{z}$.

Analogamente, $\mathbf{S O}(2)$ e $\mathbf{S}^{1}$ são isomorfos e a ação definida em (1.4) juntamente com a ação padrão de $\mathbf{S O}(2)$ definida em (1.2) são isomorfas.

\subsubsection{Integral de Haar}

Todo grupo de Lie compacto $\Gamma$ agindo num espaço vetorial de dimensão $n$ pode ser identificado com um subgrupo fechado do $\mathbf{O}(n)$ para algum $n$. Esse fato é provado através da integral de Haar que definimos a seguir.

Definição 1.1.7. Considere $f: \Gamma \rightarrow \mathbb{R}$ uma função continua. A operação $\int_{\gamma \in \Gamma} f(\gamma)$, ou $\int_{\Gamma} f$, ou $\int_{\Gamma} f d \gamma$ é uma integral de Haar em $\Gamma$ se satizfaz:

a) Linearidade: $\int_{\Gamma}(a f+b g)=a \int_{\Gamma} f+b \int_{\Gamma} g$, onde $f, g: \Gamma \rightarrow \mathbb{R}$ são contínuas e $a, b \in \mathbb{R}$;

b) Positividade: Se $f(\gamma) \geqslant 0$, para todo $\gamma \in \Gamma$, então $\int_{\Gamma} f \geqslant 0$;

c) Invariância por translação: $\int_{\Gamma} f(\delta \gamma)=\int_{\Gamma} f(\gamma)$, para toto $\delta \in \Gamma$ fixado.

Para grupos compactos, toda integral de Haar invariante à esquerda também é invariante à direita, isto é,

$$
\int_{\Gamma} f(\gamma \delta)=\int_{\Gamma} f(\gamma)
$$

Se $\Gamma$ é um grupo de Lie compacto, então $\Gamma$ é limitado e portanto $\int_{\Gamma} 1$ é finito. Assim podemos sempre assumir a integral de Haar normalizada: $\int_{\Gamma} 1=1$.

Observação 1.1.8. Se $\Gamma$ é um grupo de Lie finito de ordem $|\Gamma|$, então a integral de Haar normalizada em $\Gamma$ é dada por

$$
\int_{\Gamma} f \equiv \frac{1}{|\Gamma|} \sum_{\gamma \in \Gamma} f(\gamma)
$$

Usando a integral de Haar construímos um produto interno $\Gamma$-invariante $\langle,\rangle_{\Gamma}$ em $V$, ou seja,

$$
\langle\gamma v, \gamma w\rangle_{\Gamma}=\langle v, w\rangle_{\Gamma}, \forall \gamma \in \Gamma, \mathrm{e} v, w \in V
$$

de modo que podemos identificar $\Gamma$ com um subgrupo fechado do $\mathbf{O}(n)$ e daí assumir $\Gamma$ como um grupo de Lie compacto agindo ortogonalmente em $V$. Isto é dado pelo teorema a seguir: 
Teorema 1.1.9. Seja $\Gamma$ um grupo de Lie compacto agindo num espaço vetorial $V$. Então existe um produto interno em $V$ tal que, para todo $\gamma \in \Gamma$, a representação $\rho_{\gamma} e ́$ ortogonal.

Demonstração: Seja $\langle\rangle:, V \rightarrow \mathbb{R}$ um produto interno em $V$. Para $v, w \in V$ definimos

$$
\langle v, w\rangle_{\Gamma}=\int_{\Gamma}\langle\gamma v, \gamma w\rangle
$$

Da linearidade e da positividade da integral de Haar segue que (1.9) é um produto interno. Dados $v, w \in V$, defina $f: \Gamma \rightarrow \mathbb{R}$ por $f(\gamma)=\langle\gamma v, \gamma w\rangle$ contínua. Para cada $\gamma \in \Gamma$, temos

$$
\begin{gathered}
\left.\left.\langle\gamma v, \gamma w\rangle_{\Gamma}=\int_{\alpha \in \Gamma}\langle\alpha(\gamma v), \alpha(\gamma w)\rangle=\int_{\alpha \in \Gamma}\langle(\alpha \gamma) v),(\alpha \gamma) w\right)\right\rangle= \\
\int_{\alpha \in \Gamma} f(\alpha \gamma)=\int_{\alpha \in \Gamma} f(\alpha)=\langle v, w\rangle_{\Gamma},
\end{gathered}
$$

mostrando que esse produto interno em $V$ é invariante sob a ação de $\Gamma$. Como $V$ é um espaço vetorial real segue

$$
\langle v, u\rangle_{\Gamma}=\left\langle\rho_{\gamma} v, \rho_{\gamma} u\right\rangle_{\Gamma}=\left\langle v, \rho_{\gamma} \rho_{\gamma}^{t} u\right\rangle_{\Gamma}
$$

para todo $u \in V$. Logo $\rho_{\gamma} \rho_{\gamma}^{t}=I_{n \times n}$ implicando que $\rho_{\gamma}$ é ortogonal para todo $\gamma \in \Gamma$.

\subsection{Irredutibilidade}

Nessa seção descrevemos a decomposição de uma representação de um grupo de Lie compacto $\Gamma$ em uma soma direta de representações mais simples chamadas irredutiveis, sendo fundamentais para a teoria de representação. Apesar dessa decomposição não ser única, mostramos que ela sempre existe.

Seja $\Gamma$ um grupo de Lie agindo linearmente em um espaço vetorial real $V$ finitamente gerado.

Definição 1.2.1. a) Um subespaço $W \subseteq V$ é $\Gamma$-invariante se $\gamma w \in W, \forall \gamma \in \Gamma$;

b) Uma representação ou ação de $\Gamma$ em $V$ é irredutivel se os únicos subespaços $\Gamma$ invariantes de $V$ são os triviais $\{0\}$ e $V$;

c) Um subespaço $W \subseteq V$ é $\Gamma$-irredutivel se $W$ é $\Gamma$-invariante e a ação de $\Gamma$ em $W$ é irredutivel.

Exemplo 1.2.2. A ação de $\mathbf{O}(2)$ em $\mathbb{C}$ definida em (1.6), é irredutível para $k \neq 0$. Geometricamente, a ação corresponde a rotações e reflexões no plano. Então os únicos subespaços que podem ser preservados com a ação de todas essas transformações são os subespaços triviais.

O complementar de um subespaço $\Gamma$-invariante ainda é $\Gamma$-invariante: 
Proposição 1.2.3. Seja $\Gamma$ um grupo de Lie compacto agindo em $V$ de dimensão finita, e $W \subseteq V$ um subespaço $\Gamma$-invariante. Então existe um subepaço complementar $Z$, $\Gamma$-invariante tal que $V=W \oplus Z$.

Demonstração: Seja $Z=W^{\perp}=\left\{v \in V:\langle v, w\rangle_{\Gamma}=0, \forall w \in W\right\}$ sendo $\langle,\rangle_{\Gamma} \mathrm{o}$ produto interno definido em (1.9). Devido à invariância de $W$, temos que, para cada $\gamma \in \Gamma, \rho_{\gamma}: W \rightarrow W$ é um isomorfismo. Logo, dado $w \in W$, existe $w_{1} \in W$ tal que $w=\rho_{\gamma} w_{1}=\gamma w_{1}$. Se $v \in W$, segue da invariância do produto interno que

$$
\langle\gamma v, w\rangle_{\Gamma}=\left\langle\gamma v, \gamma w_{1}\right\rangle_{\Gamma}=\left\langle v, w_{1}\right\rangle=0, \forall w \in W
$$

implicando que $\gamma v \in W^{\perp}, \forall \gamma \in \Gamma$ e portanto $Z=W^{\perp}$ é $\Gamma$-invariante.

Como consequência da proposição acima, qualquer representação de um grupo de Lie compacto pode ser escrita como uma soma direta de subespaços irredutíveis:

Corolário 1.2.4. Seja $\Gamma$ um grupo de Lie compacto agindo em V. Então existem subespaços $\Gamma$-invariantes e irredutiveis $V_{1}, \ldots, V_{s}$ tais que

$$
V=V_{1} \oplus \ldots \oplus V_{s}
$$

Demonstração: Podemos assumir $\mathrm{V}$ não nulo. Então $V$ contém um subespaço $\Gamma$ irredutível. Seja $V_{1}$ o subespaço não nulo de menor dimensão $\Gamma$-invariante.

Pela proposição anterior, existe um subespaço complementar $Z \subset V, \Gamma$-invariante, tal que $V=V_{1} \oplus Z$. Aplicando esse processo em $Z$ encontramos um subespaço $V_{2}$ nas mesmas condições de $V_{1}$. Como a dimensão de $V$ é finita o processo termina com a decomposição de $\Gamma$ em uma soma direta de subespaços $\Gamma$-invariantes e irredutíveis.

Exemplo 1.2.5. Seja $V=\mathbb{M}_{2 \times 2}(\mathbb{R})$ e considere a ação padrão de $\mathbf{S O}(2)$ em $V$ dada por

$$
R_{\theta} \cdot A=R_{\theta} A
$$

Considerando $V_{1}$ e $V_{2}$ os subespaços de $\mathbb{M}_{n \times n}(\mathbb{R})$ dos elementos respectivamente da forma

$$
\left(\begin{array}{ll}
a & 0 \\
b & 0
\end{array}\right) \text { e }\left(\begin{array}{ll}
0 & c \\
0 & d
\end{array}\right)
$$

com $a, b, c, d \in \mathbb{R}$ temos $V=V_{1} \oplus V_{2}$ onde $V_{1}$ e $V_{2}$ são $\mathbf{S O}(2)$-irredutíveis. Além disso, também temos $V=V_{1} \oplus V_{3}$ sendo $V_{3}$ subespaço $\mathbf{S O}(2)$-irredutível, dado por

$$
V_{3}=\left(\begin{array}{cc}
8 c & c \\
8 d & d
\end{array}\right)
$$

ilustrando que a decomposição de $V$ dada em (1.10) não é única.

Teorema 1.2.6. Seja $\Gamma$ um grupo de Lie compacto agindo em $V$.

a) $A$ menos de $\Gamma$-isomorfismo existe um número finito de subespaços distintos de $V$ $\Gamma$-irredutiveis. Denotemos por $U_{1}, \ldots, U_{t}$. 
b) Seja $W_{k}$ a soma de todos os subepaços $W \subset V$-irredutiveis tal que $W$ é $\Gamma$-isomorfo a $U_{k}$ para algum $k \in\{1, \ldots, t\}$. Então,

$$
V=W_{1} \oplus \ldots \oplus W_{t} .
$$

A prova do teorema acima será dada mais abaixo.

Os subespaços $W_{k}$ são chamados de componentes isotípicas de $V$ e a decomposição acima é chamada de decomposição isotípica.

Corolário 1.2.7. Seja $\Gamma$ um grupo de Lie compacto agindo em $V$.

a) $S e W \subset V$ é $\Gamma$ - irredutível, então $W \subset W_{k}$ para um único $k$, para o qual $W$ e $U_{k}$ são $\Gamma$-isomorfos.

b) Seja $V=V_{1} \oplus \ldots \oplus V_{s}$ a decomposição de $V$ numa soma direta de subespaços $\Gamma$ invariantes e irredutiveis. Se as representações de $\Gamma$ em $V_{j}$ são todas distintas (sem $\Gamma$-isomorfismos), então os únicos subespaços não nulos $\Gamma$-irredutiveis de $V$ são $V_{1}, V_{2}, \ldots, V_{s}$.

\section{Demonstração:}

a) Suponha $W \subset W_{i} \cap W_{j}$. Então $W$ é $\Gamma$-isomorfo aos subespaços $U_{i}$ e $U_{j}$, com $i \neq j$. Logo $U_{i}$ e $U_{j}$ também são $\Gamma$-isomorfos, o que é absurdo.

b) Considere as componentes isotípicas $W_{k}$ de $V$. Cada $V_{j} \subset W_{k}$ para um único $k$ pois são $\Gamma$ - irredutíveis. Observe que não existe $V_{i}, V_{j} \subset W_{k} \operatorname{com} i \neq j$ pois não são $\Gamma$-isomorfos. Logo as componentes isotípicas de $V$ são os próprios $V_{j}^{\prime} s$. Além disso, se $U \neq\{0\}$ é subespaço $\Gamma$-irredutível de $\mathrm{V}$, então $U \subset W_{k}=V_{j}$ para algum $j$. Como $V_{j}$ é $\Gamma$ - irredutível temos $U=V_{j}$. Portanto, os únicos subespaços não nulos $\Gamma$ - irredutíveis de $V$ são $V_{1}, V_{2}, \ldots, V_{s}$.

A prova do Teorema 1.2.6 depende de dois lemas, os quais apresentamos a seguir.

Lema 1.2.8. Seja $\Gamma$ um grupo de Lie compacto agindo em $W$ de dimensão finita. Suponha que

$$
W=\sum_{\alpha} U_{\alpha}
$$

onde cada $U_{\alpha}$ é um subespaço $\Gamma$ - invariante e $\Gamma$-isomorfo para alguma representação irredutivel $U$ de $\Gamma$. Então, todo subespaço $\Gamma$-irredutivel de $W$ é $\Gamma$-isomorfo a $U$.

Demonstração: Mostremos que existe um conjunto finito de índices tal que

$$
W=U_{\alpha_{1}} \oplus \ldots \oplus U_{\alpha_{s}} .
$$

Temos $W_{1}=U_{\alpha_{1}} \subset W$. Se $W_{1}=W$ então o processo termina. Caso contrário, existe $U_{\alpha_{2}} \subset W$ tal que $U_{\alpha_{2}} \nsubseteq W_{1}$. Além disso $W_{1} \cap U_{\alpha_{2}} \subset U_{\alpha_{2}}$ é $\Gamma$ - invariante. Pela irredutibilidade de $U_{\alpha_{2}}$ temos $W_{1} \cap U_{\alpha_{2}}=\{0\}$. Portanto, $W_{2}=U_{\alpha_{1}} \oplus U_{\alpha_{2}} \subset W$ é uma 
soma direta de $U_{\alpha_{1}}$ e $U_{\alpha_{2}}$. Se $W_{2}=W$ segue o resultado. Caso contrário repetimos o processo. Como a dimensão de $W$ é finita o processo termina com a obtenção de (1.13).

Agora mostremos que se $X \neq\{0\}$ é um subespaço $\Gamma$-irredutível de $W$, então $X$ é $\Gamma$-isomorfo ao subespaço $U$.

Dado $X \neq\{0\}$, existe um único $t \leq s$ tal que

$$
X \nsubseteq U_{\alpha_{1}} \oplus \ldots \oplus U_{\alpha_{t-1}}
$$

e

$$
X \subset U_{\alpha_{1}} \oplus \ldots \oplus U_{\alpha_{t}} .
$$

De fato, como $X$ é $\Gamma$ - irredutível, então $X \nsubseteq U_{\alpha_{1}}$, o qual também é $\Gamma$-irredutível pois é $\Gamma$ isomorfo a um subespaço $U$ de $W$ que por sua vez é $\Gamma$ - irredutível. Então, se $X \subset U_{\alpha_{1}} \oplus U_{\alpha_{2}}$ o processo termina. Caso contrário, se $X \nsubseteq U_{\alpha_{1}} \oplus U_{\alpha_{2}}$ repetimos o mesmo argumento. Dessa forma, o processo termina com a obtenção de (1.14) e (1.15).

Além disso

$$
X \cap U_{\alpha_{1}} \oplus \ldots \oplus U_{\alpha_{t-1}}=\{0\},
$$

pois $U_{\alpha_{1}} \oplus \ldots \oplus U_{\alpha_{t-1}}$ é um subespaço $\Gamma$-invariante de $X$. Considere a projeção

$$
\pi: U_{\alpha_{1}} \oplus \ldots \oplus U_{\alpha_{t}} \rightarrow U_{\alpha_{t}} .
$$

Observe agora que $\pi_{\left.\right|_{X}}: X \rightarrow \pi(X)$ é linear e sobrejetora e por (1.16) também é injetora (pois o núcleo dessa aplicação é nula). Portanto, $\pi_{\left.\right|_{X}}: X \rightarrow \pi(X)$ é um isomorfismo linear. Ainda, se $\gamma \in \Gamma$ e $x=x_{\alpha_{1}} \oplus \ldots \oplus x_{\alpha_{t}} \in X$, onde $x_{\alpha_{t}} \neq 0$ então $\pi_{\left.\right|_{X}}(\gamma x)=\gamma \pi_{\left.\right|_{X}}(x)$.

Como $\pi(X) \neq\{0\}$ e $\pi(X) \subset U_{\alpha_{t}}$ implica que $\pi(X)=U_{\alpha_{t}}$ pela irredutibilidade de $U_{\alpha_{t}}$, temos que $X$ é $\Gamma$-isomorfo a $U_{\alpha_{t}}$ e, portanto, a $U$.

Lema 1.2.9. Seja $\Gamma$ um grupo de Lie compacto agindo em $V$. Sejam $X$ e $Y$ subespaços de $V \Gamma$-invariantes tal que não existem dois subespaços $\Gamma$-irredutiveis $W \subset X$ e $Z \subset Y$ com $W$ Г- isomorfo a $Z$. Então:

a) $X \cap Y=\{0\}$;

b) $S e W \subset X \oplus Y$ é $\Gamma$ - irredutivel temos $W \subset X$ ou $W \subset Y$.

Demonstração:

a) Se $X$ e $Y$ são $\Gamma$ - invariantes então $X \cap Y$ é $\Gamma$-invariante. Então qualquer subespaço $\Gamma$ - irredutível $A \subset X \cap Y$ também é $\Gamma$ - irredutível de $X$ e $Y$. Pela hipótese do teorema tal fato ocorre apenas para $X \cap Y=\{0\}$. Provando assim a primeira parte.

b) Seja $W \subset X \oplus Y \Gamma$-irredutível. Da invariância de $X$ e $Y$ segue que $W \cap X$ e $W \cap Y$, subespaços de $W$, também são $\Gamma$ - invariantes. Pela irredutibilidade de $W$ segue que $W \cap X=\{0\}$ ou $W \subset X$ e $W \cap Y=\{0\}$ ou $W \subset Y$. Dessas possibilidades suponha $W \nsubseteq X, W \nsubseteq Y$ e $W \cap X=W \cap Y=\{0\}$ e considere as projeções $\pi_{x}: X \oplus Y \rightarrow X$ e $\pi_{y}: X \oplus Y \rightarrow Y$. Analogamente à demonstração do Lema 1.2.8, $W \subset X \oplus Y$ é $\Gamma$-isomorfo a $\pi_{x}(W)$ e $\pi_{y}(W)$ e, portanto, $\pi_{x}(W)$ e $\pi_{y}(W)$ são $\Gamma$ - isomorfos, contrariando a hipótese do Lema. Então $W \subset X$ ou $W \subset Y$. 
Demonstração do Teorema 1.2.6: Escolha um subespaço $\Gamma$-irredutível $U_{1} \subset V$ e seja $W_{1}^{\prime}$ a soma de todos os subespaços $\Gamma$-invariantes de $V$ e $\Gamma$ - isomorfo a $U_{1}$. Então $W_{1}^{\prime}$ também é $\Gamma$ - invariante. Se $W_{1}^{\prime} \neq V$ tomamos o complementar invariante $W_{1}^{\prime \perp}$ de $W_{1}^{\prime}$ obtendo $V=W_{1}^{\prime} \oplus W_{1}^{\prime \perp}$ e repetimos o processo em $W_{1}^{\prime \perp}$.

Sejam $U_{2} \subset W_{1}^{\perp \perp}$ subespaço $\Gamma$ - irredutível e $W_{2}^{\prime}$ a soma de todos os subespaços $\Gamma$ invariantes de $W_{1}^{\prime \perp}$ e $\Gamma$-isomorfo a $U_{2}$. Se $W_{2}^{\prime} \neq W_{1}^{\prime \perp}$, tomamos o complementar de $W_{2}^{\prime}$, obtendo $W_{1}^{\prime \perp}=W_{2}^{\prime} \oplus W_{2}^{\prime \perp}$ e repetimos o processo em $W_{2}^{\prime \perp}$. Como a dimensão de $V$ é finita o processo termina com

$$
V=W_{1}^{\prime} \oplus \ldots \oplus W_{t}^{\prime}
$$

onde cada $W_{k}^{\prime}$ é a soma de subespaços $\Gamma$ - irredutíveis de $V$ e $\Gamma$-isomorfos a $U_{k}$. E se $i \neq j$, então $U_{i}$ e $U_{j}$ não são $\Gamma$-isomorfos.

Seja $U \subset V$ subespaço $\Gamma$-irredutível. Pelo Lema 1.2.9(b) temos $U \subset W_{k}^{\prime}$ para algum $k$ e pelo Lema 1.2.8 $U$ é $\Gamma$-isomorfo a $U_{k}$. Portanto, a menos de $\Gamma$-isomorfismo, existe um número finito de subespaços distintos de $V$.

Observe agora que $W_{k}^{\prime}=W_{k}$, onde $W_{k}$ são as componentes isotípicas definidas no Teorema 1.2.6(b). De fato, pois por construção todo irredutível de $V$ está contido em $W_{k}^{\prime}$ para algum $k$ e, portanto, $W_{k}=W_{k}^{\prime}$.

Por construção a decomposição isotípica é única.

\subsection{Germes de funções invariantes e de aplicações equivariantes}

Nesta seção damos uma descrição de germes de aplicações que comutam com a ação de um grupo de Lie compacto $\Gamma$. Começamos com a teoria de invariantes e apresentamos dois resultamos de extrema importância: O Teorema de Hilbert-Weyl, que garante a existência de uma base finita para o anel das funções polinomiais invariantes pela ação de $\Gamma$, e o Teorema de Schwarz (Schwarz [1975]), mostrando que essa base também gera o anel dos germes invariantes sob a ação de $\Gamma$.

Definimos germe de uma aplicação:

Definição 1.3.1. Seja $x \in \mathbb{R}^{n}$ e seja $\mathfrak{F}$ o conjunto de todas aplicações $C^{\infty} f: U \subset$ $\mathbb{R}^{n} \rightarrow \mathbb{R}^{m}$, definida em uma vizinhaça $U$ de $x$. Em $\mathfrak{F}$ definimos a seguinte classe de equivalência: $f_{1}: U_{1} \subset \mathbb{R}^{n} \rightarrow \mathbb{R}^{m}$ e $f_{2}: U_{2} \subset \mathbb{R}^{n} \rightarrow \mathbb{R}^{m}$ são equivalentes se existe um aberto $W \subset U_{1} \cap U_{2}$ contendo $x$ tal que $f_{\left.\right|_{\mid}}=f_{\left.2\right|_{W}}$. A classe de equivalência da aplicação $f$ é chamada de germe de $f$ em $x$, ou simplesmente de germe de $f$.

Se $y=f(x)$, chamamos $x$ de fonte do germe e $y$ de meta do germe. Denotamos por $\mathcal{E}_{n, m}$ o conjunto dos germes de funções com fonte nula, isto é, $\mathcal{E}_{n, m}=\left\{f:\left(\mathbb{R}^{n}, 0\right) \rightarrow \mathbb{R}^{m}\right\}$. Se $m=1$, denotamos apenas por $\mathcal{E}_{n}$. 


\subsubsection{Germes de funções invariantes}

Definição 1.3.2. Seja $\Gamma$ um grupo de Lie compacto agindo num espaço vetorial $V$. Dizemos que um germe de função $f:(V, 0) \rightarrow \mathbb{R}$ é $\Gamma$-invariante se

$$
f(\gamma x)=f(x)
$$

para todo $\gamma \in \Gamma$ e $x \in V$.

De forma análoga definimos polinômios de $V \rightarrow \mathbb{R} \Gamma$-invariantes.

Observação 1.3.3. Pela linearidade da ação de $\Gamma$ é suficiente verificar a igualdade (1.18) para o grupo $\Gamma$ apenas para os geradores de $\Gamma$.

Exemplos 1.3.4. 1. Sendo $\Gamma$ um grupo de Lie compacto agindo num espaço de dimensão $n$, podemos assumir $\Gamma \subseteq \mathbf{O}(n)$ pelo Teorema 1.1.9. Neste caso, a norma $\|x\|=x_{1}^{2}+\ldots+x_{n}^{2}$ é sempre $\Gamma$-invariante. De fato,

$$
\|\gamma x\|^{2}=\langle\gamma x, \gamma x\rangle_{\Gamma}=\langle x, x\rangle_{\Gamma}=\|x\|^{2} .
$$

2. Seja $\Gamma=\mathbf{Z}_{2}=\{-1,1\}$ agindo em $V=\mathbb{R}$ pela ação padrão. Se $p: \mathbb{R} \rightarrow \mathbb{R}$ é um polinômio $\mathbf{Z}_{2}$-invariante, então $p(-x)=p(x)$ para todo $x \in V$ e, portanto, $p$ é um polinômio par. Logo, existe um polinômio $h: \mathbb{R} \rightarrow \mathbb{R}$ tal que $p(x)=h\left(x^{2}\right)$.

3. Seja $\Gamma=\mathbf{S}^{1}$ agindo em $V=\mathbb{C}$ pela ação definida no Exemplo 1.1.5(3). Isto é,

$$
\begin{aligned}
\mathbf{S}^{1} \times \mathbb{C} & \rightarrow \mathbb{C} \\
(\theta, z) & \mapsto e^{i \theta} z .
\end{aligned}
$$

Se $p$ é um polinômio $\mathbf{S}^{1}$-invariante em $\mathbb{C}$, então existe um polinômio $h: \mathbb{R} \rightarrow \mathbb{R}$ tal que

$$
p(z)=h(z \bar{z}) .
$$

Geometricamente, essa aplicação transforma um elemeto $z$ em um círculo $C$ de raio $|z|$ centrado na origem. Então, se $f: \mathbb{C} \rightarrow \mathbb{R}$ é $\mathbf{S}^{1}$-invariante, $f$ é constante em $C$.

Seja $p: \mathbb{R} \rightarrow \mathbb{R}$ um polinômio $\mathbf{S}^{1}$-invariante. Podemos escrever $p$ nas coordenadas $z, \bar{z} \in \mathbb{C}$ na forma

$$
p(z)=\sum a_{\alpha \beta} z^{\alpha} \bar{z}^{\beta}, a_{\alpha \beta} \in \mathbb{C} .
$$

Como $p(z)=p\left(e^{i \theta} z\right)$ para todo $\theta \in \mathbf{S}^{1}$ e $z \in \mathbb{C}$, temos

$$
\sum a_{\alpha \beta} z^{\alpha} \bar{z}^{\beta}=\sum a_{\alpha \beta} e^{i \theta(\alpha-\beta)} z^{\alpha} \bar{z}^{\beta}, \forall \theta \in \mathbf{S}^{1} .
$$

Então $a_{\alpha \beta}={ }_{\alpha \beta} e^{i \theta(\alpha-\beta)}$, para todo $\theta \in \mathbf{S}^{1}$. Logo $a_{\alpha \beta}=0$ ou $\alpha=\beta$. Portanto,

$$
p(z)=\sum a_{\alpha}(z \bar{z})^{\alpha}
$$

$\operatorname{com} a_{\alpha}, z \in \mathbb{C}$. Como $p$ é polinômio real temos $p=\bar{p}$. Então,

$$
\sum a_{\alpha}(z \bar{z})^{\alpha}=\sum \bar{a}_{\alpha}(z \bar{z})^{\alpha}
$$

implicando que $a_{\alpha}=\bar{a}_{\alpha}$ e, portanto, $a_{\alpha} \in \mathbb{R}$. Considerando o polinômio $h: \mathbb{R} \rightarrow \mathbb{R}$ tal que $h(x)=\sum a_{\alpha} x^{\alpha}$, segue (1.19). 
4. Considere o grupo diedral $\mathbf{D}_{n}$ agindo no espaço $V=\mathbb{C}$ com a ação padrão definida no Exemplo 1.1.5,(6). Se $p$ é um polinômio $\mathbf{D}_{n}$-invariante, existe um polinômio $h: \mathbb{R}^{2} \rightarrow \mathbb{R}$ tal que

$$
p(z)=h\left(z \bar{z}, z^{n}+\bar{z}^{n}\right) .
$$

De fato, seja $p: \mathbb{C} \rightarrow \mathbb{R}$ polinômio $\mathbf{D}_{n}$-invariante. Então podemos escrevê-lo nas coordenadas $z, \bar{z} \in \mathbb{C}$ como em (1.20). Observe que $\mathbf{D}_{n}$ é gerado por $\xi=2 \pi / n \mathrm{e}$ pela conjugação complexa $\kappa$. Da invariância de $p$ em relação a $\kappa$, temos

$$
p(z)=p(\kappa z)=p(\bar{z})=\sum \bar{a}_{\alpha \beta} z^{\beta} \bar{z}^{\alpha},
$$

mostrando que

$$
a_{\alpha \beta}=a_{\beta \alpha} .
$$

Como $p=\bar{p}$, concluímos que $a_{\alpha \beta}=\bar{a}_{\beta \alpha}$ e, portanto, $a_{\alpha \beta} \in \mathbb{R}$. Agora, da invariância de $p$ em relação a $\xi$, obtemos

$$
\sum a_{\alpha \beta} z^{\alpha} \bar{z}^{\beta}=\sum a_{\alpha \beta} e^{i \xi(\alpha-\beta)} z^{\alpha} \bar{z}^{\beta},
$$

e portanto,

$$
a_{\alpha \beta} e^{i \xi(\alpha-\beta)} z^{\alpha} \bar{z}^{\beta}=a_{\alpha \beta} z^{\alpha} \bar{z}^{\beta}, \operatorname{com} \xi=2 \pi / n .
$$

Consequentemente,

$$
\begin{gathered}
a_{\alpha \beta}=0, \text { ou } \\
\alpha \equiv \beta(\bmod n), \quad \text { isto é, } \alpha-\beta=k n, k \in \mathbb{Z} .
\end{gathered}
$$

Usando as expressões acima escrevemos $p$ na forma

$$
p(z)=\sum A_{\alpha \beta}\left(z^{\alpha} \bar{z}^{\beta}+z^{\beta} \bar{z}^{\alpha}\right),
$$

onde

$$
A_{\alpha \beta}= \begin{cases}a_{\alpha \beta} & \text { se } \alpha \neq \beta, \\ \frac{a_{\alpha \beta}}{2} & \text { se } \alpha=\beta .\end{cases}
$$

Fatorando a equação (1.25) e utilizando (1.23) e (1.24) chegamos a

$$
p(z)=\sum_{j, k} B_{j k}(z \bar{z})^{j}\left(z^{k n}+\bar{z}^{k n}\right),
$$

para certos $B_{j k} \in \mathbb{R}$. Finalmente, usando a identidade

$$
z^{k n}+\bar{z}^{k n}=\left(z^{n}+\bar{z}^{n}\right)\left(z^{(k-1) n}+\bar{z}^{(k-1) n}\right)-z \bar{z}\left(z^{(k-2) n}+\bar{z}^{(k-2) n}\right),
$$

indutivamente sobre $k$, escrevemos o polinômio $p$ na forma

$$
p(z)=\sum_{l, m} C_{l m}(z \bar{z})^{l}\left(z^{n}+\bar{z}^{n}\right)^{m}
$$

para certos reais $C_{l m}$. Definindo $h: \mathbb{R}^{2} \rightarrow \mathbb{R}$ tal que $h(x, y)=\sum C_{l m} x^{l} y^{m}$, obtemos (1.22). 
Existe um subconjunto finito de polinômios $\Gamma$-invariantes $u_{1}, \ldots, u_{s}$ tal que todo polinômio $\Gamma$-invariante pode ser escrito em função de $u_{1}, \ldots, u_{s}$. Esse conjunto, o qual não é único, é chamado de conjunto gerador dos polinômios invariantes, ou simplesmente base de Hilbert, e sua existência é dada pelo teorema de Hilbert-Weyl que enunciamos a seguir.

Se $\Gamma$ age num espaço $V$, denotamos por $\mathcal{P}_{V}(\Gamma)$ o anel dos polinômios $\Gamma$ - invariantes e por $\mathcal{E}_{V}(\Gamma)$ o anel dos germes de funções $(V, 0) \rightarrow \mathbb{R} \Gamma$ - invariantes. É fácil ver que $\mathcal{P}_{V}(\Gamma)$ e $\mathcal{E}_{V}(\Gamma)$ são de fato anéis sobre $\mathbb{R}$, pois soma e produto de invariantes ainda é um invariante. Se $u_{1}, \ldots, u_{s}$ é uma base de Hilbert para $\mathcal{P}_{V}(\Gamma)$ denotamos $\mathcal{P}_{V}(\Gamma)=$ $\left\langle u_{1}, \ldots, u_{s}\right\rangle$. Quando não houver dúvida em qual espaço $\Gamma$ está agindo, denotamos os anéis dos invariantes apenas por $\mathcal{P}(\Gamma)$ e por $\mathcal{E}(\Gamma)$.

Teorema 1.3.5. (Hilbert - Weyl) Seja $\Gamma$ um grupo de Lie compacto agindo em $V$. Então existem $u_{1}, \ldots, u_{s} \in \mathcal{P}(\Gamma)$ que geram o anel $\mathcal{P}(\Gamma)$ sobre $\mathbb{R}$.

Demonstração: Veja [15], XII, Teorema 4.2.

Sendo assim, observamos nos Exemplos 1.3.4 o seguinte:

1. $\mathcal{P}_{\mathbb{R}}\left(\mathbf{Z}_{2}\right)=\left\langle x^{2}\right\rangle ;$

2. $\mathcal{P}_{\mathbb{C}}\left(\mathbf{S}^{1}\right)=\langle z \bar{z}\rangle$;

3. $\mathcal{P}_{\mathbb{C}}\left(\mathbf{D}_{n}\right)=\left\langle z \bar{z}, z^{n}+\bar{z}^{n}\right\rangle$.

Teorema 1.3.6. (Schwarz [1975]) Seja $\Gamma$ um grupo de Lie compacto agindo em $V e$ sejam $u_{1}, \ldots, u_{s}$ uma base de Hilbert para $\mathcal{P}_{V}(\Gamma)$. Se $f \in \mathcal{E}_{V}(\Gamma)$, então existe um germe suave $h: \mathbb{R}^{s} \rightarrow \mathbb{R}$ tal que

$$
f(x)=h\left(u_{1}(x), \ldots, u_{s}(x)\right) .
$$

Demonstração: Veja [15], XII, §6(b).

Em outas palavras, o Teorema de Schwarz garante que se $u_{1}, \ldots, u_{s}$ é uma base de Hilbert para $\mathcal{P}_{V}(\Gamma)$ então esses mesmos elementos formam uma base de Hilbert para $\mathcal{E}_{V}(\Gamma)$.

Dizemos que o anel $\mathcal{P}(\Gamma)$ admite uma relação se existe um polinômio não nulo $r: \mathbb{R}^{s} \rightarrow \mathbb{R}$ tal que

$$
r\left(u_{1}(x), \ldots, u_{s}(x)\right) \equiv 0,
$$

onde $\left\{u_{1}, \ldots, u_{s}\right\}$ é uma base de Hilbert para $\mathcal{P}(\Gamma)$.

Definição 1.3.7. Um anel é chamado polinomial se ele admite uma base de Hilbert sem relaçôes.

Um exemplo de uma ação de grupo para a qual $\mathcal{P}(\Gamma)$ não é um anel polinomial é dada por $\Gamma=\mathbf{Z}_{2}$ agindo em $\mathbb{R}^{2}$, onde a ação de $-1 \in \mathbf{Z}_{2}$ é dada por $-1(x, y)=(-x,-y)$. As funções polinomiais

$$
u_{1}=x_{1}^{2}, \quad u_{2}=x_{1} x_{2}, \quad u_{3}=x_{2}^{2}
$$

formam uma base de Hilbert para $\mathcal{P}\left(\mathbf{Z}_{2}\right)$, porém existe a relação $u_{1} u_{3}-u_{2}^{2} \equiv 0$. É fácil ver que $\mathcal{P}\left(\mathbf{Z}_{2}\right)$ de fato não admite base sem relações. 
Existe um teste simples para determinar quando $\mathcal{P}(\Gamma)$ é um anel polinomial. Dado $u_{1}, \ldots, u_{s}$ base de Hilbert para $\mathcal{P}(\Gamma)$, defina

$$
\begin{aligned}
\rho: V & \rightarrow \mathbb{R}^{s} \\
x & \mapsto\left(u_{1}(x), \ldots, u_{s}(x)\right),
\end{aligned}
$$

chamada de discriminante de $\Gamma$.

Lema 1.3.8. Para $\rho$ dado em (1.28), se o Jacobiano $(d \rho)_{x}$ é sobrejetor para algum $x$, então $\mathcal{P}(\Gamma)$ é um anel polinomial.

Demonstração: Se $(d \rho)_{x}$ é sobrejetora para algum $x \in V$, então pelo Teorema da Função Implícita $\rho(V)$ contém um aberto do $\mathbb{R}^{s}$ onde toda aplicação polinomial $r: \mathbb{R}^{s} \rightarrow \mathbb{R}$ é determinada por $r_{\rho_{\rho(V)}}$. Agora suponha $r\left(u_{1}(x), \ldots, u_{s}(x)\right) \equiv 0$. Então $r_{\rho_{\rho(V)}} \equiv 0$ e portanto, $r \equiv 0$ implicando que não existe relações para $u_{1}, \ldots u_{s}$ em $\mathcal{P}(\Gamma)$, o que o torna um anel polinomial.

Para ilustrar este lema, retomemos o Exemplo 1.3.4(4). Temos $u_{1}=z \bar{z}$ e $u_{2}=z^{n}+\bar{z}^{n}$ base de Hilbert para $\mathcal{P}_{\mathbb{C}}\left(\mathbf{D}_{n}\right)$. Então $\rho(z)=\left(z \bar{z}, z^{n}+\bar{z}^{n}\right)$, com

$$
d \rho=\left(\begin{array}{cc}
\bar{z} & z \\
n z^{n-1} & n \bar{z}^{n-1}
\end{array}\right) .
$$

Logo $\operatorname{det}(d \rho)_{z}=n\left(\bar{z}^{n}-z^{n}\right) \neq 0$, para todo $z^{n} \neq \bar{z}^{n}$. Segue do Lema 1.3 .8 que $\mathcal{P}_{\mathbb{C}}\left(\mathbf{D}_{n}\right)$ é um anel polinomial.

\subsubsection{Germes de aplicações equivariantes}

Sejam $(\rho, V)$ e $(\eta, W)$ representações de um grupo de Lie compacto $\Gamma$.

Definição 1.3.9. Um germe de aplicação $g: V \rightarrow W$ comuta com $\Gamma$, ou é $\Gamma$-equivariante, se

$$
g\left(\rho_{\gamma} v\right)=\eta_{\gamma} g(v), \forall \gamma \in \Gamma, v \in V
$$

Se $(\rho, V)=(\eta, W)$, escrevemos apenas $g(\gamma v)=\gamma g(v)$.

Com essa definição, podemos reformular a Definição 1.1.6 dizendo que duas representações $(\rho, V)$ e $(\eta, W)$ são $\Gamma$-isomorfas, se existe um isomorfismo linear $A: V \rightarrow W$ $\Gamma$-equivariante.

Lema 1.3.10. (Lema de Schur) Sejam $(\rho, V)$ e $(\eta, W)$ representações irredutiveis de $\Gamma e \varphi: V \rightarrow W$ um germe de aplicação linear $\Gamma$-equivariante. Então, ou $\varphi$ é invertivel ou é identicamente nula.

Demonstração: Veja [6], II, Theorem 1.10.

Definição 1.3.11. Uma representação $(\rho, V)$ de um grupo $\Gamma$ em $V$ é absolutamente irredutivel se os únicos germes de aplicações em $V \Gamma$-equivariantes são as múltiplas da identidade. 
Lema 1.3.12. Seja $\Gamma$ um grupo de Lie compacto agindo em $V$. Se a ação de $\Gamma$ é absolutamente irredutivel, então ela é irredutivel.

Demonstração: Suponha que a ação de $\Gamma$ não seja irredutível. Então existe um subespaço não nulo $W \subsetneq V, \Gamma$ - invariante com seu complementar $W^{\perp}$ também $\Gamma$ invariante tal que $V=W \oplus W^{\perp}$. Defina $\pi: W \oplus W^{\perp} \rightarrow W$ a projeção sobre $W$. Para qualquer $v=v_{1}+v_{2} \in V$, com $v_{1} \in W$ e $v_{2} \in W^{\perp}$, temos

$$
\pi(\gamma v)=\pi\left(\gamma v_{1}+\gamma v_{2}\right)=\gamma v_{1}=\gamma \pi(v), \forall \gamma \in \Gamma .
$$

Logo $\pi$ é $\Gamma$ - equivariante e não é múltipla da identidade e, portanto, $V$ não é absolutamente irredutível.

Lema 1.3.13. Seja $\Gamma$ um grupo de Lie compacto agindo em $V$. Seja $A: V \rightarrow V$ um germe de aplicação linear $\Gamma$-equivariante e $W \subset V$ um subespaço $\Gamma$-irredutível. Então:

a) $A(W)$ é $\Gamma$-invariante;

b) $A(W)=\{0\}$ ou as representações de $\Gamma$ em $W$ e $A(W)$ são $\Gamma$-isomorfas.

Demonstração:

a) Seja $v=A(w) \in A(W)$. Pela equivariância de $A$ segue que

$$
\gamma v=\gamma A(w)=A(\gamma w) \in A(W), \forall \gamma \in \Gamma .
$$

Logo $A(W)$ é $\Gamma$-invariante.

b) Para qualquer $v \in \operatorname{ker}(A)$, temos

$$
A(\gamma v)=\gamma A(v)=0, \forall \gamma \in \Gamma .
$$

Logo $\operatorname{ker}(A)$ também é $\Gamma$-invariante. Então $\operatorname{ker}(A) \cap W \subset W$ também é $\Gamma$ - invariante e pela irredutibilidade de $W$ segue que $\operatorname{ker}(A) \cap W=\{0\}$ ou $\operatorname{ker}(A) \cap W=W$. Se $\operatorname{ker}(A) \cap W=W, \operatorname{temos} W \subset \operatorname{ker}(A)$ e, então, $A(W)=\{0\}$. Se $\operatorname{ker}(A) \cap W=$ $W, A: W \rightarrow A(W)$ é um isomorfismo linear $\Gamma$ - equivariante e, portanto, $W$ e $A(W)$ são $\Gamma$-isomorfos.

Esse lema implica diretamente no teorema a seguir:

Teorema 1.3.14. Seja $\Gamma$ um grupo de Lie compacto agindo em $V$. Decomponha $V$ em componentes isotípicas, isto é,

$$
V=W_{1} \oplus \ldots \oplus W_{s}
$$

Se $A: V \rightarrow V$ é um germe de aplicação linear $\Gamma$-equivariante, então $A\left(W_{k}\right) \subset W_{k}$, para todo $k=1, \ldots, s$. 
Demonstração: Pelo Lema 1.2.8 podemos escrever $W_{k}$ como

$$
W_{k}=V_{1} \oplus \ldots \oplus V_{r}
$$

onde cada $V_{j}$ é $\Gamma$ isomorfo ao $\Gamma$-irredutível $U_{k}$ e, portanto, também é $\Gamma$-irredutível. Do Lema 1.3.13, segue que $A\left(V_{j}\right)$ é $\Gamma$-invariante e $A\left(V_{j}\right)=\{0\}$ ou $A\left(V_{j}\right)$ é $\Gamma$-isomorfo a $V_{j}$ e então $\Gamma$-isomorfo a $U_{k}$. Em qualquer um dos casos temos $A\left(V_{j}\right) \subset W_{k}$, para todo $j=1, \ldots, r$. Logo $A\left(W_{k}\right) \subset W_{k}$, para todo $k=1, \ldots, s$.

Lema 1.3.15. Se $f: V \rightarrow \mathbb{R}$ é $\Gamma$-invariante e $g: V \rightarrow W$ é $\Gamma$-equivariante, então $f g: V \rightarrow W$ é $\Gamma$-equivariante.

Demonstração: Para todo $x \in V$ e $\gamma \in \Gamma$ temos

$$
\begin{aligned}
(f g)\left(\rho_{\gamma} x\right) & =f\left(\rho_{\gamma} x\right) g\left(\rho_{\gamma} x\right) \\
& =f(x) \eta_{\gamma} g(x) \\
& =\eta_{\gamma} f(x) g(x) \\
& =\eta_{\gamma} f g(x) .
\end{aligned}
$$

Denotamos por $\overrightarrow{\mathcal{E}}_{V, W}(\Gamma)$ o conjunto de todos or germes $C^{\infty}$ de $(V, 0) \rightarrow W$ $\Gamma$-equivariantes, e por $\overrightarrow{\mathcal{P}}_{V, W}(\Gamma)$ o conjunto de todas as aplicações polinomiais $V \rightarrow W \Gamma$ equivariantes. Se $V=W$ escrevemos apenas $\overrightarrow{\mathcal{E}}_{V}(\Gamma)$ e $\overrightarrow{\mathcal{P}}_{V}(\Gamma)$. E quando não houver dúvidas sobre qual espaço $\Gamma$ está agindo escreveremos apenas $\overrightarrow{\mathcal{E}}(\Gamma)$ e $\overrightarrow{\mathcal{P}}(\Gamma)$.

É fácil ver que $\overrightarrow{\mathcal{E}}_{V, W}(\Gamma)$ e $\overrightarrow{\mathcal{P}}_{V, W}(\Gamma)$ são espaços vetoriais sobre $\mathbb{R}$. O lema anterior implica que $\overrightarrow{\mathcal{E}}_{V, W}(\Gamma)$ e $\overrightarrow{\mathcal{P}}_{V, W}(\Gamma)$ são módulos sobre $\mathcal{E}_{V}(\Gamma)$ e $\mathcal{P}_{V}(\Gamma)$, respectivamente.

Definição 1.3.16. Dizemos que $g_{1}, \ldots, g_{r}$ geram o módulo $\overrightarrow{\mathcal{P}}_{V, W}(\Gamma)$ sobre o anel $\mathcal{P}_{V}(\Gamma)$ se, para todo $g \in \overrightarrow{\mathcal{P}}_{V, W}(\Gamma)$, existem $f_{1}, \ldots, f_{r} \in \mathcal{P}_{V}(\Gamma)$ tal que

$$
g=f_{1} g_{1}+\ldots+f_{r} g_{r}
$$

De modo análogo define-se conjunto gerador para o módulo $\overrightarrow{\mathcal{E}}_{V, W}(\Gamma)$ sobre o anel $\mathcal{E}_{V}(\Gamma)$

Nos exemplos a seguir encontramos um conjunto de geradores para $\overrightarrow{\mathcal{P}}_{V, W}(\Gamma)$ sobre o anel $\mathcal{P}_{V}(\Gamma)$ :

Exemplos 1.3.17. 1. Considere a ação padrão de $\Gamma=\mathbf{S}^{1}$ em $V=\mathbb{C}$, isto é, para todo $\theta \in \mathbf{S}^{1}, z \in \mathbb{C}, \theta z=e^{i \theta} z$. Seja $g: \mathbb{C} \rightarrow \mathbb{C}$ uma aplicação polinomial $\Gamma$-equivariante. Então

$$
g(z)=p(z \bar{z}) z+q(z \bar{z}) i z,
$$

onde $p, q \in \mathcal{P}\left(\mathbf{S}^{1}\right)$. Vamos escrever $g$ nas coordenadas $z, \bar{z} \in \mathbb{C}$ na forma

$$
g(z)=\sum b_{j k} z^{j} \bar{z}^{k}
$$


$\operatorname{com} b_{j k} \in \mathbb{C}$. Mas $g(\theta z)=\theta g(z)$ implica em

$$
\begin{aligned}
g(z) & =e^{-i \theta} g\left(e^{i \theta} z\right) \\
& =e^{-i \theta} \sum b_{j k}\left(e^{i \theta} z\right)^{j}\left(\overline{e^{i \theta} z}\right)^{k} \\
& =\sum b_{j k} e^{i \theta(j-k-1)} z^{j} \bar{z}^{k} .
\end{aligned}
$$

Comparando com (1.31) temos

$$
b_{j k}=b_{j k} e^{i \theta(j-k-1)}, \forall \theta \in \mathbf{S}^{1},
$$

implicando que $b_{j k}=0$ ou $j-k-1=0$. Então,

$$
g(z)=\sum b_{j k}(z \bar{z})^{k} z .
$$

Como $b_{j k} \in \mathbb{C}$, temos $b_{j k}=c_{j k}+d_{j k} i, \operatorname{com} c_{j k}, d_{j k} \in \mathbb{R}$. Logo,

$$
g(z)=\sum c_{j k}(z \bar{z})^{k} z+\sum d_{j k}(z \bar{z})^{k} z i .
$$

Se considerarmos $p(x)=\sum c_{j k} x^{k}$ e $q(x)=\sum d_{j k} x^{k}$, temos

$$
g(z)=p(z \bar{z}) z+q(z \bar{z}) i z
$$

com $p, q \in \mathcal{P}\left(\mathbf{S}^{1}\right)$ e $z, z i \in \overrightarrow{\mathcal{P}}\left(\mathbf{S}^{1}\right)$. Portanto,

$$
\overrightarrow{\mathcal{P}}\left(\mathbf{S}^{1}\right)=\mathcal{P}\left(\mathbf{S}^{1}\right)\{z, z i\} .
$$

2. Seja $\Gamma=\mathbf{O}(2)$ agindo em $\mathbb{C}$ com a ação padrão, definida em (1.6) para $k=1$. Se $g: \mathbb{C} \rightarrow \mathbb{C}$ é uma aplicação polinomial $\Gamma$-equivariante, então

$$
g(z)=p(z \bar{z}) z
$$

Para mostrar esse fato poderíamos usar uma construção análoga ao exemplo anterior. Porém notemos que, em particular, $g$ é $\mathbf{S}^{1}$ - equivariante, então $g(z)=$ $p(z \bar{z}) z+q(z \bar{z}) i z$. Logo,

$$
\overline{g(\bar{z})}=\overline{p(z \bar{z}) \bar{z}}+\overline{q(z \bar{z}) i \bar{z}}=p(z \bar{z}) z-q(z \bar{z}) z i .
$$

Mas $g(\kappa z)=\kappa g(z)$, implicando em $g(z)=\overline{g(\bar{z})}$. Segue daí que $q(z \bar{z})=0$. Logo, $g(z)=p(z \bar{z}) z$ como queríamos mostrar.

3. Considere o grupo diedral $\Gamma=\mathbf{D}_{n}$ agindo em $V=\mathbb{C}$, com a ação padrão definida no Exemplo 1.1.5(6). Toda aplicação $g \in \overrightarrow{\mathcal{P}}(\Gamma)$ é da forma

$$
g(z)=p(u, v) z+q(u, v) \bar{z}^{n-1},
$$

onde $u=z \bar{z}$ e $v=z^{n}+\bar{z}^{n}$. Para deduzir (1.33) seguimos as idéias dos outros exemplos, escrevendo $g$ na forma

$$
g(z)=\sum b_{j k} z^{j} \bar{z}^{k}, b_{j k} \in \mathbb{C} .
$$


O grupo $\mathbf{D}_{n}$ é gerado pela rotação $\xi=2 \pi / n$ juntamente com a conjugação complexa $\kappa$. Segue da equivariância de $g$ em relação a $\kappa$ que

$$
g(z)=\overline{g(\bar{z})}=\sum \bar{b}_{j k} z^{j} \bar{z}^{k}
$$

e então $b_{j k} \in \mathbb{R}$. Agora, pela equivariância em relação a $\xi=2 \pi / n$ segue que

$$
g(z)=\sum b_{j k} e^{i \xi(j-k-1)} z^{j} \bar{z}^{k}
$$

Então,

$$
\begin{gathered}
b_{j k}=0, \text { ou } \\
j \equiv k(\bmod n), \quad \text { isto é, } j-k=\ln , l \in \mathbb{Z} .
\end{gathered}
$$

Mostremos agora que $z$ e $\bar{z}^{n-1}$ geram o módulo $\overrightarrow{\mathcal{P}}\left(\mathbf{D}_{n}\right)$ sobre $\mathcal{P}\left(\mathbf{D}_{n}\right)$. Podemos fatorar (1.34) em termos de $z \bar{z}$, os quais são $\mathbf{D}_{n^{-}}$invariantes, até chegarmos em $j=0$ ou $k=0$, como, por exemplo, $z^{j} \bar{z}^{k}=(z \bar{z})^{j} \bar{z}^{k-j}$ se $k \geq j$, ou $z^{j} \bar{z}^{k}=(z \bar{z})^{k} \bar{z}^{j-k}$ se $j \geq k$.

Desde que $j \equiv k+1(\bmod n)$, os termos $z^{l n+1}$ e $\bar{z}^{l n+n-1}=\bar{z}^{(l+1) n-1}$ para $l=$ $0,1,2, \ldots$. Note que para $l \geq 2$ temos as identidades:

a) $z^{(l+2) n+1}=\left(z^{n} \bar{z}^{n}\right) z^{(l+1) n+1}-(z \bar{z})^{n} z^{l n+1} ;$
b) $\bar{z}^{(l+3) n-1}=\left(z^{n} \bar{z}^{n}\right) \bar{z}^{(l+2) n-1}-(z \bar{z}) \bar{z}^{(l+1) n-1}$

e, portanto, os geradores $z^{l n+1}$ e $\bar{z}^{(l+1) n-1}$ são redutíveis para $l \geq 2$. Para $l=1$ temos,

a) $z^{n+1}=\left(z^{n} \bar{z}^{n}\right) z-(z \bar{z}) \bar{z}^{n-1}$

b) $\bar{z}^{2 n-1}=\left(z^{n} \bar{z}^{n}\right) \bar{z}^{n-1}-(z \bar{z})^{n-1} \bar{z}$.

Ou seja, para $l=0$ os geradores ainda são redutíveis. Assim, os termos $z$ e $\bar{z}^{n-1}$ correspondentes à $l=0$ geram $\overrightarrow{\mathcal{P}}\left(\mathbf{D}_{n}\right)$.

O teorema abaixo, encontrado em [15], é a versão equivariante dos teoremas de Hilbert-Weyl e Schwarz.

Teorema 1.3.18. (Poénaru [1976]) Seja $\Gamma$ um grupo de Lie compacto agindo nos espaços $V$ e $W$.

a) O módulo $\overrightarrow{\mathcal{P}}_{V, W}(\Gamma)$ é finitamente gerado sobre o anel $\mathcal{P}_{V}(\Gamma)$.

b) Sejam $g_{1}, \ldots, g_{r}$ geradores para o módulo $\overrightarrow{\mathcal{P}}_{V, W}(\Gamma)$ sobre o anel $\mathcal{P}_{V}(\Gamma)$. Então $g_{1}, \ldots, g_{r}$ também geram o módulo $\overrightarrow{\mathcal{E}}_{V, W}(\Gamma)$ sobre o anel $\mathcal{E}_{V}(\Gamma)$.

Antes de darmos uma prova para este teorema fazemos algumas observações. Esse teorema nos mostra que, para obtermos um conjunto gerador para o módulo dos germes equivariantes, é suficiente encontrarmos geradores para o módulo das aplicações polinomiais equivariantes. Dessa forma concluímos a partir dos Exemplos 1.3.17 os seguintes resultados: 

1. $\overrightarrow{\mathcal{E}}\left(\mathbf{S}^{1}\right)=\mathcal{E}\left(\mathbf{S}^{1}\right)\{z, z i\}$
2. $\overrightarrow{\mathcal{E}}(\mathbf{O}(2))=\mathcal{E}(\mathbf{O}(2))\{z\}$
3. $\overrightarrow{\mathcal{E}}\left(\mathbf{D}_{n}\right)=\mathcal{E}\left(\mathbf{D}_{n}\right)\left\{z, \bar{z}^{n-1}\right\}$.

Definição 1.3.19. Dizemos que $g_{1}, \ldots, g_{r}$ geram livremente o módulo $\overrightarrow{\mathcal{E}}_{V, W}(\Gamma)$ sobre o anel $\mathcal{E}_{V}(\Gamma)$ se a relação $f_{1} g_{1}+\ldots+f_{r} g_{r} \equiv 0$, com $f_{j} \in \mathcal{E}_{V}(\Gamma)$ implica $f_{1} \equiv \ldots \equiv f_{r} \equiv 0$.

Neste caso dizemos que $\overrightarrow{\mathcal{E}}_{V, W}(\Gamma)$ é um módulo livre sobre $\mathcal{E}_{V}(\Gamma)$.

De forma análoga definimos para $\overrightarrow{\mathcal{P}}_{V, W}(\Gamma)$.

Se $g_{1}, \ldots, g_{r}$ geram livremente o módulo $\overrightarrow{\mathcal{E}}_{V, W}(\Gamma)$ sobre $\mathcal{E}_{V}(\Gamma)$ então para todo $g \in$ $\overrightarrow{\mathcal{E}}_{V, W}(\Gamma)$, existem únicos $f_{j} \in \mathcal{E}_{V}(\Gamma)$ tais que

$$
g=f_{1} g_{1}+\ldots+f_{r} g_{r}
$$

De fato, se $g=f_{1} g_{1}+\ldots+f_{r} g_{r}=h_{1} g_{1}+\ldots+h_{r} g_{r}$, então $\left(f_{1}-h_{1}\right) g_{1}+\ldots+\left(f_{r}-h_{r}\right) g_{r} \equiv 0$. Como o módulo é livre, segue que $f_{j}=h_{j}, \forall j=1, \ldots, r$.

Todos os módulos que vimos até esse momento são livres. Vejamos no próximo exemplo o grupo diedral agindo em $\mathbb{C}$ :

Exemplo 1.3.20. O módulo $\overrightarrow{\mathcal{E}}\left(\mathbf{D}_{n}\right)$ é livre sobre o anel $\mathcal{E}\left(\mathbf{D}_{n}\right)$. Suponha $p(u, v) z+$ $q(u, v) \bar{z}^{n-1} \equiv 0$. Se $q$ não é a função nula, então numa vizinhança em $\mathbb{C}$ temos $\frac{p(u, v)}{q(u, v)} z+$ $\bar{z}^{n-1} \equiv 0$ e, portanto, $\frac{p(u, v)}{q(u, v)} z \bar{z}+\bar{z}^{n} \equiv 0$. Mas $\frac{p(u, v)}{q(u, v)} z \bar{z}$ é sempre real, enquanto que $\bar{z}^{n}$ não. Assim, necessariamente $q \equiv 0$. $\operatorname{Logo} p(u, v) z=0$, para todo $z \in \mathbb{C}$, implicando em $p(u, v) \equiv 0$.

Agora provamos um lema que será uma importante ferramenta para a prova do teorema de Poénaru. A idéia aqui é converter o caso equivariante para um contexto invariante.

Lema 1.3.21. Sejam $(\rho, V)$ e $(\eta, W)$ representações de um grupo de Lie compacto $\Gamma$, e $g: V \rightarrow W \Gamma$-equivariante. Então existe uma aplicação $f: V \times W \rightarrow \mathbb{R}$ tal que

$$
g(x)=\left(d_{y} f\right)_{(x, 0)}^{t}
$$

Demonstração: Sejam $n$ e $m$ as dimensões de $V$ e $W$ respectivamente, então $g(x)=$ $\left(g_{1}(x), \ldots, g_{m}(x)\right)$ e denotemos $y=\left(y_{1}, \ldots, y_{m}\right) \in W$. Induzimos uma ação de $\Gamma$ em $V \times W$, dada por $\gamma \cdot(x, y)=\left(\rho_{\gamma} x, \eta_{\gamma} y\right)$. Como $\Gamma$ é compacto, existe um produto interno $\Gamma$-invariante $\langle$,$\rangle em V \times W$, construído em (1.9). Defina $f: V \times W \rightarrow \mathbb{R}$ por $f(x, y)=\langle g(x), y\rangle$. Então, $f$ é $\Gamma$-invariante. De fato,

$$
f(\gamma \cdot(x, y))=f\left(\rho_{\gamma} x, \eta_{\gamma} y\right)=\left\langle g\left(\rho_{\gamma} x\right), \eta_{\gamma} y\right\rangle=\left\langle\rho_{\gamma} g(x), \eta_{\gamma} y\right\rangle=\langle g(x), y\rangle .
$$

Temos

$$
\frac{\partial f}{\partial y_{j}}=\left\langle d_{y_{j}} g(x), y\right\rangle+\left\langle g(x), d_{y_{j}} y\right\rangle .
$$


Por exemplo, para $j=1$, temos

$$
\begin{aligned}
d_{y_{1}} f_{(x, 0)} & =\left\langle d_{y_{1}} g, y\right\rangle_{(x, 0)}+\left\langle g(x), d_{y_{1}} y\right\rangle_{(x, 0)} \\
& =\langle 0, y\rangle_{(x, 0)}+\left\langle\left(g_{1}(x), \ldots, g_{m}(x)\right),(1,0, \ldots, 0)\right\rangle_{(x, 0)}=0+g_{1}(x)=g_{1}(x) .
\end{aligned}
$$

De forma análoga obtemos

$$
d_{y_{j}} f_{(x, 0)}=g_{j}(x), \forall j=1, \ldots, m \text {. }
$$

Então

$$
g(x)=\left(d_{y_{1}} f_{(x, 0)}, \ldots, d_{y_{m}} f_{(x, 0)}\right)=\left(d_{y} f\right)_{(x, 0)}^{t}
$$

Em geral, o germe de aplicação $g: V \rightarrow W$ definida em (1.36) é $\Gamma$-equivariante desde que $f: V \times W \rightarrow \mathbb{R}$ seja $\Gamma$-invariante. De fato, se $f(x, y)=f\left(\rho_{\gamma} x, \eta_{\gamma} y\right)$ segue da regra da cadeia que

$$
\left(d_{y} f\right)_{(x, 0)}=\left(d_{y} f\right)_{\left(\rho_{\gamma} x, 0\right)} \rho_{\gamma},
$$

e então

$$
\left(d_{y} f\right)_{(x, 0)}^{t}=\rho_{\gamma}^{t}\left(d_{y} f\right)_{\left(\rho_{\gamma} x, 0\right)}^{t} .
$$

Mas pelo Teorema 1.1.9 a ação de $\Gamma$ é ortogonal e sendo assim $\rho_{\gamma}^{t}=\rho_{\gamma}^{-1}$. Logo, $g(x)=\rho_{\gamma}^{-1} g\left(\rho_{\gamma} x\right)$, e então $\rho_{\gamma} g(x)=g\left(\rho_{\gamma} x\right)$, mostrando que de fato $g$ é $\Gamma$-equivariante. I

\section{Demonstração do Teorema 1.3.18:}

a) Suponha $g: V \rightarrow W \Gamma$-equivariante e defina $f$ como no Lema 1.3 .21 anterior. Podemos recuperar a $\Gamma$-equivariância de $g$ pela relação

$$
g(x)=\left(d_{y} f\right)_{(x, 0)}^{t}
$$

Como $f \in \mathcal{P}_{V \times W}(\Gamma)$, segue pelo Teorema de Schwarz que existe $h: \mathbb{R}^{m+n} \rightarrow \mathbb{R}$, tal que $f=h\left(u_{1}(x, y), \ldots, u_{s}(x, y)\right)$, com $\left\{u_{1}, \ldots, u_{s}\right\}$ base de Hilbert de $\mathcal{P}_{V \times W}(\Gamma)$.

Então,

$$
d_{y} f=\frac{\partial f}{\partial u_{1}}\left(u_{1}, \ldots, u_{s}\right) d_{y} u_{1}(x, y)+\ldots+\frac{\partial f}{\partial u_{s}}\left(u_{1}, \ldots, u_{s}\right) d_{y} u_{s}(x, y) .
$$

Segue das expressões (1.38) e (1.39) que

$$
g(x)=\frac{\partial f}{\partial u_{1}}\left(u_{1}, \ldots, u_{s}\right)_{(x, 0)}\left(d_{y} u_{1}\right)_{(x, 0)}^{t}+\ldots+\frac{\partial f}{\partial u_{s}}\left(u_{1}, \ldots, u_{s}\right)_{(x, 0)}\left(d_{y} u_{s}\right)_{(x, 0)}^{t} .
$$

Para todo $j \in\{1, \ldots, s\}$, temos $\frac{\partial f}{\partial u_{j}}\left(u_{1}, \ldots, u_{s}\right)_{(x, 0)} \in P_{V}(\Gamma)$ pois $u_{1}, \ldots, u_{s} \in$ $\mathcal{P}_{V \times W}(\Gamma)$, e temos $\left(d_{y} u_{j}\right)_{(x, 0)}^{t} \in \overrightarrow{\mathcal{P}}_{V, W}(\Gamma)$ como vimos no lema acima.

Concluímos que $\left(d_{y} u_{1}\right)_{(x, 0)}^{t}, \ldots,\left(d_{y} u_{s}\right)_{(x, 0)}^{t}$ geram o módulo $\overrightarrow{\mathcal{P}}_{V, W}(\Gamma)$ sobre o anel $\mathcal{P}_{V}(\Gamma)$. 
b) Observe que se $g$ é um germe de aplicação, então $f$ também é um germe e pelo Teorema de Schwarz 1.3.6 $\left\{u_{1}, \ldots, u_{s}\right\}$ é também base de Hilbert para $\mathcal{E}_{V \times W}(\Gamma)$. Como a construção que fizemos foi independente da natureza de $f$, obtemos o mesmo conjunto gerador para o módulo $\overrightarrow{\mathcal{E}}_{V, W}(\Gamma)$ sobre o anel $\mathcal{E}_{V}(\Gamma)$.

Esses cálculos nos mostram que a forma geral dos germes de aplicações $\Gamma$-equivariantes em $\overrightarrow{\mathcal{E}}_{V, W}(\Gamma)$ podem ser obtidas a partir da construção feita no Lema 1.3.21 através de um germe de função $\Gamma$-invariante em $\mathcal{E}_{V \times W}(\Gamma)$. Assim é suficiente conhecermos uma base de Hilbert para $\mathcal{P}_{V \times W}(\Gamma)$.

\section{4 Órbitas e subgrupos de isotropia}

Seja $\Gamma$ um grupo de Lie compacto agindo em $V$.

\subsection{1 Órbitas, isotropias e subespaço de ponto fixo}

Definição 1.4.1. Definimos a órbita da ação de $\Gamma$ em $x \in V$ como

$$
\Gamma_{x}=\{\gamma x: \gamma \in \Gamma\}
$$

Se $g: V \rightarrow W$ é $\Gamma$-equivariante, então $g(x)=0$ se, e somente se, $g$ se anula na órbita de $x$. Em outras palavras, zeros de germes de aplicações equivariantes aparecem ao longo de toda a órbita de um zero $x \in V$.

Definição 1.4.2. Definimos o subgrupo de isotropia de $x \in V$ como

$$
\Sigma_{x}=\{\gamma \in \Gamma: \gamma x=x\} .
$$

Definição 1.4.3. Seja $\Sigma$ subgrupo de $\Gamma$. O subespaço de ponto fixo de $\Sigma$ é dado por

$$
\operatorname{Fix}_{V} \Sigma=\{x \in V: \gamma x=x, \forall \gamma \in \Sigma\}
$$

Escrevemos apenas $\operatorname{Fix}(\Sigma)$ quando estiver subtendido qual é o espaço em que $\Gamma$ está agindo.

Observe que $\operatorname{Fix}_{V}(\Sigma)$ é sempre um subespaço vetorial de $V$, pois

$$
\operatorname{Fix}_{V}(\Sigma)=\bigcap_{\gamma \in \Sigma} \operatorname{ker}\left(\rho_{\gamma}-i d\right)
$$

Lema 1.4.4. Pontos em uma mesma órbita de $\Gamma$ têm subgrupos de isotropia conjugados. Mais precisamente,

$$
\Sigma_{x}=\gamma \Sigma_{x} \gamma^{-1}
$$

Demonstração: Seja $\gamma \sigma \gamma \in \gamma \Sigma_{x} \gamma^{-1}$. Então,

$$
\gamma \sigma \gamma(\gamma x)=\gamma \sigma(x)=\sigma(x)=x,
$$


pois $\sigma \in \Sigma_{x}$. Logo $\gamma \sigma \gamma \in \Sigma_{\gamma x}$, para todo $\sigma \in \Sigma_{x}$ e, portanto, $\gamma \Sigma_{x} \gamma \subset \Sigma_{\gamma x}$.

Com o mesmo raciocício, observamos que

$$
\gamma^{-1} \Sigma_{\gamma x} \gamma \subset \Sigma_{\gamma^{-1}(\gamma x)}=\Sigma_{x}
$$

Então, para todo $\sigma \in \Sigma_{\gamma x}$, temos $\gamma^{-1} \sigma \gamma=\beta \in \Sigma_{x}$. Segue que $\sigma=\gamma \beta \gamma^{-1} \in \gamma \Sigma_{x} \gamma^{-1} \mathrm{e}$ daí $\Sigma_{\gamma x} \subset \gamma \Sigma_{x} \gamma^{-1}$. Portanto $\Sigma_{x}=\gamma \Sigma_{x} \gamma^{-1}$.

Definição 1.4.5. Um subgrupo de isotropia $\Sigma \subset \Gamma$ é maximal se não existe um subgrupo de isotropia $\Delta \subset \Gamma$ satisfazendo $\Sigma \nsubseteq \Delta \nsubseteq \Gamma$.

Lema 1.4.6. Seja $\operatorname{Fix}(\Gamma)=\{0\}$ e $\Sigma \subset \Gamma$. Então $\Sigma$ é um subgrupo de isotropia maximal se, e somente se, $\operatorname{dim} \operatorname{Fix}(\Sigma)>0$ e $\operatorname{dim} \operatorname{Fix}(\Delta)=0$, para todo subgrupo fechado $\Delta \subset \Gamma$ tal que $\Delta \nsupseteq \Sigma$.

Demonstração: Seja $\operatorname{Fix}(\Gamma)=\{0\}$ e $\Sigma$ um subgrupo de isotropia maximal. Direto da hipótese temos $\operatorname{dim} \operatorname{Fix}(\Sigma)>0$. Seja $\Delta$ subgruppo fechado tal que $\Delta \supsetneq \Sigma$. Se $x \in \operatorname{Fix}(\Delta)$ então $\delta x=x \forall \delta \in \Delta$. Logo $\Sigma_{x} \supset \Delta \supsetneq \Sigma$. Mas $\Sigma$ é maximal e, portanto, $\Sigma_{x}=\Gamma$. Como $\operatorname{Fix}(\Gamma)=0, \operatorname{temos} x=0$ e, portanto, $\operatorname{dim} \operatorname{Fix}(\Delta)=0$.

Por outro lado, $\operatorname{dim} \operatorname{Fix}(\Sigma)>0$, mostrando que existe $x \in V$ não nulo tal que $x \in \operatorname{Fix}(\Sigma)$. Então $\Sigma \subset \Sigma_{x}$. Suponha $\Sigma \neq \Sigma_{x}$. Por hipótese, $\operatorname{dim} \operatorname{Fix}\left(\Sigma_{x}\right)=0$. Absurdo pois $x \in \operatorname{Fix}\left(\Sigma_{x}\right)$. Logo $\Sigma=\Sigma_{x}$ mostranto que $\Sigma$ é um subgrupo de isotropia de $\Gamma$. Mostremos que é maximal. Seja $\Delta$ um subgrupo de isotropia tal que $\Delta \supseteq \Sigma$. Por hipótese $\operatorname{dim} \operatorname{Fix}(\Delta)=0$. Mas $\Delta=\Sigma_{y}$ para algum $y \in V$, implicando que $y \in \operatorname{Fix}(\Delta)$ e portanto $y=0$. $\operatorname{Logo} \Delta=\Sigma_{0}=\Gamma$.

Para descrever geometricamente a ação de um grupo $\Gamma$ em $V$ é conveniente olhar para o conjunto $W$ formado por todos os pontos de $V$ que têm subgrupo de isotropia conjugados. Dizemos que $W$ é um "tipo de órbita " desta ação.

Ilustramos essas idéias considerando a ação padrão de $\mathbf{D}_{n}$ em $V=\mathbb{C}$ dada por

$$
\begin{aligned}
& \xi \cdot z=e^{i \xi} z \\
& \kappa \cdot z=\bar{z} .
\end{aligned}
$$

Geometricamente identificamos a ação do grupo $\mathbf{D}_{n}$ como sendo as simetrias que preservam o poligono regular de $n$ lados, centrado na origem. O tipo de órbita depende da paridade de $n$. Consideremos $n=5$. Então $\mathbf{D}_{5}$ é o grupo das simetrias que preservam o pentágono centrado na origem. (Ver figura 1.1).

1. Os vértices do pentágono constituem uma única órbita na ação de $\Gamma$, então pelo Lema 1.4.4, os subgrupos de isotropia de todos os vértices são conjugados. Observe ainda que para qualquer vértice o tamanho de sua órbita é $2 n$.

2. Se $t \in \mathbb{R}, t \neq 0$, então $\Sigma_{t z}=\Sigma_{z}$, mostrando que todos os pontos, exceto a origem, que pertencem às retas que passam por um vértice e pela origem têm o mesmo subgrupo de isotropia. 


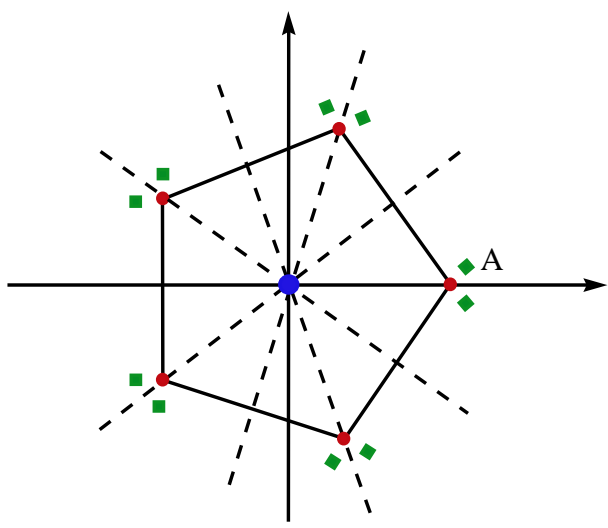

Figura 1.1: Órbitas da ação do $\mathbf{D}_{5}$ em $\mathbb{C}$.

3. Se considerarmos um ponto próximo ao eixo real, como por exemplo, o ponto $A$ da figura 1.1, então sua órbita também tem $2 n=10$ elementos.

4. O subgrupo de isotropia de qualquer ponto no eixo real, exceto a origem, é dado por $\mathbf{Z}_{2}$.

5. A origem forma uma órbita e tem como subgrupo de isotropia todo o $\mathbf{D}_{5}$.

Concluímos que $\mathbb{C}$ possui três tipos de órbitas para $\mathbf{D}_{5}$. Análise análoga vale para todo $n \geq 3$, n ímpar. Na tabela abaixo listamos os tipos de órbitas para todo $n$ ímpar, $n \geq 3$.

\begin{tabular}{c|c|c} 
Tipo de órbita & Subgrupo de Isotropia & Tamanho da órbita \\
\hline$\{0\}$ & $\mathbf{D}_{n}$ & 1 \\
\hline$\left\{z \in \mathbb{C}: I m z^{n}=0: z \neq 0\right\}$ & $\mathbf{Z}_{2}$ & $n$ \\
\hline$\left\{z \in \mathbb{C}: I m z^{n} \neq 0\right\}$ & $\{I\}$ & $2 n$ \\
\hline
\end{tabular}

Tabela 1.1: Tipos de órbitas e subgrupo de isotropia para o grupo $\mathbf{D}_{n}$ agindo em $\mathbb{C}$, com $n$ ímpar, $n \geq 3$.

Pela Tabela 1.1 observamos uma relação entre o tamanho da órbita e do subgrupo de isotropia. Essa relação será formalizada na próxima proposição.

Proposição 1.4.7. Seja $\Gamma$ grupo de Lie compacto agindo em $V$.

a) Se $\Gamma$ é finito, então $|\Gamma|=\left|\Sigma_{x}\right|\left|\Gamma_{x}\right|$ sendo $x \in V$.

b) $\operatorname{dim} \Gamma=\operatorname{dim} \Sigma_{x}+\operatorname{dim} \Gamma_{x}$

Demonstração:

a) Defina

$$
\begin{aligned}
\psi: \Gamma / \Sigma_{x} & \rightarrow \Gamma_{x} \\
\gamma \Sigma_{x} & \mapsto \gamma x
\end{aligned}
$$


Note que $\psi$ está bem definida, pois se $\gamma \Sigma_{x}=\gamma_{2} \Sigma_{x}$, então $\Sigma_{x}=\gamma_{1}^{-1} \gamma_{2} \Sigma_{x}$, e daí $\gamma_{1}^{-1} \gamma_{2} \in \Sigma_{x}$. Logo, $\gamma_{1}^{-1} \gamma_{2} x=x$, e então $\gamma_{1} x=\gamma_{2} x$. Para todo $\beta \in \Gamma_{x}$ existe $\gamma \in \Gamma$ tal que $\beta=\gamma x$. Então $\beta=\psi\left(\gamma \Sigma_{x}\right)$, mostrando que $\psi$ é sobrejetor. Note que $\psi$ também é injetora, pois se $\gamma x=\gamma_{1} x$, então $x=\gamma^{-1} \gamma_{1} x$, implicando que $\gamma^{-1} \gamma_{1} \in \Sigma_{x}$. Logo $\Sigma_{x}=\gamma^{-1} \gamma_{1} \Sigma_{x}$ e daí, $\gamma \Sigma_{x}=\gamma_{1} \Sigma_{x}$.

Portanto, $\psi$ é uma bijeção, e então $\left|\Gamma / \Sigma_{x}\right|=\left|\Gamma_{x}\right|$. Como $\left|\Gamma / \Sigma_{x}\right|=|\Gamma|\left|\Sigma_{x}\right|$, segue o resultado.

A aplicação $\psi$ é uma aplicação diferenciável entre variedades no ponto $0 \in \Gamma / \Sigma_{x}$. Então

$$
(d \psi)_{0}: T_{0}\left(\Gamma / \Sigma_{x}\right) \rightarrow T_{\psi(0)}\left(\Gamma_{x}\right)
$$

é uma transformação linear entre espaços tangentes. Como $\psi$ é um difeomorfismo, segue que $(d \psi)_{0}$ é um isomorfismo. Logo $\operatorname{dim} T_{0}\left(\Gamma / \Sigma_{x}\right)=\operatorname{dim} T_{\psi(0)}\left(\Gamma_{x}\right) . \quad \operatorname{Em}$ geral, se $M$ é uma variedade de dimensão $n$, então $\operatorname{dim} T_{p}(M)=\operatorname{dim} M$. Segue que $\operatorname{dim} \Gamma_{x}=\operatorname{dim} \Gamma / \Sigma_{x}-\operatorname{dim} \Sigma_{x}$.

\subsubsection{Subespaço de ponto fixo e a fórmula do traço}

Nesta subseção apresentamos alguns resultados gerais dos subespaços de ponto fixo $\operatorname{Fix}(\Sigma)$.

Começamos enfatizando os subespaços de ponto fixo $\operatorname{Fix}(I)$ e $\operatorname{Fix}(\Gamma)$, onde $I$ denota o elemento identidade do grupo. Assim $\operatorname{Fix}(I)=V$, enquanto que $\operatorname{Fix}(\Gamma)$ é o subespaço de $V$ que é fixo por todo elemento de $\Gamma$, ou seja, onde $\Gamma$ age trivialmente.

Mostramos a seguir que todo subepaço de ponto fixo tem a propriedade de invariância por um germe de aplicação equivariante:

Lema 1.4.8. Sejam $(\rho, V)$ e $(\eta, W)$ representações de $\Gamma$. Se $g: V \rightarrow W$ é um germe $\Gamma$-equivariante, então

$$
g\left(\operatorname{Fix}_{V}(\Sigma)\right) \subseteq \operatorname{Fix}_{W}(\Sigma) .
$$

Demonstração: Basta mostrar que para todo $x \in \operatorname{Fix}_{V}(\Sigma)$, temos $g(x) \in \operatorname{Fix}_{W}(\Sigma)$. Dado $x \in \operatorname{Fix}_{V}(\Sigma)$ temos $\rho_{\gamma} x=x \forall \gamma \in \Sigma$, e portanto

$$
\eta_{\gamma} g(x)=g\left(\rho_{\gamma} x\right)=g(x), \forall \gamma \in \Sigma .
$$

Uma consequência imediata do Lema 1.4 .8 acima é que se $\operatorname{Fix}(\Gamma)=\{0\}$, então 0 é necessariamente fixado por qualquer germe de aplicação $\Gamma$-equivariante.

Lema 1.4.9. Para qualquer subgrupo $\Sigma$, o subespaço de ponto fixo $\operatorname{Fix}(\Sigma)$ é igual à soma $W$ de todos os subespaços $\operatorname{Fix}(\Delta)$, tal que $\Delta \supseteq \Sigma$ é um subgrupo de isotropia. Isto é,

$$
\operatorname{Fix}(\Sigma)=\bigoplus_{i} \operatorname{Fix}\left(\Delta_{i}\right),
$$

tal que $\Delta_{i}$ é um subgrupo de isotropia contendo $\Sigma$. 
Demonstração: Seja $v \in \operatorname{Fix}(\Sigma)$. Então $\Sigma_{v} \supset \Sigma$, pois se $\sigma \in \Sigma$ temos $\sigma v=v$. Note ainda que $v \in \operatorname{Fix}\left(\Sigma_{v}\right)$. Tomando $\Delta=\Sigma_{v}$, temos $v \in \operatorname{Fix}(\Delta)$ e portanto $\operatorname{Fix}(\Sigma) \subset W$, com $\Delta \subset \Sigma$ sendo subgrupo de isotropia.

Se $w \in W$ então $w=w_{1}+\ldots+w_{k}$, onde $w_{i} \in \operatorname{Fix}\left(\Delta_{i}\right)$ com $\Delta_{i}$ sendo subgrupo de isotropia contendo $\Sigma$. Como $\Sigma \subset \Delta_{i}$, temos $\sigma w_{i}=w_{i}$, para todo $\sigma \in \Sigma$. Então $w_{i} \in \operatorname{Fix}(\Sigma)$, e daí $w \in \operatorname{Fix}(\Sigma)$ e portanto $W \subset \operatorname{Fix} \Sigma$. Concluímos que $\operatorname{Fix}(\Sigma)=W$.

Proposição 1.4.10. Seja $\Gamma$ um grupo de Lie compacto agindo em V. As seguintes afirmações são equivalentes:

a) $\operatorname{Fix}(\Gamma)=\{0\}$;

b) Toda aplicação $g: V \rightarrow V$ C-equivariante satisfaz $g(0)=0$;

c) A única função $\Gamma$-invariante linear é a função nula.

Demonstração: $(a \Rightarrow b)$ Sai direto do Lema 1.4.8.

$(b \Rightarrow a)$ Seja $v \in \operatorname{Fix}(\Gamma)$ e seja $g: V \rightarrow V$ a aplicação constante dada por $g(x)=v$. Como $g$ é $\Gamma$ - equivariante, pois $g(\gamma x)=v=\gamma v=\gamma g(x)$, temos $g(0)=0$. Mas $g(0)=v$. Portanto, $\operatorname{Fix}(\Gamma)=\{0\}$.

$(a \Rightarrow c)$ Seja $f: V \rightarrow \mathbb{R}$ uma função linear $\Gamma$-invariante. Pelo Teorema de Representação de Riesz (Veja [12]), existe $v \in V$ tal que $f(x)=\langle v, x\rangle_{\Gamma}$. Como $f(x)=f\left(\gamma^{-1} x\right)$ temos $\langle v, x\rangle_{\Gamma}=\left\langle v, \gamma^{-1} x\right\rangle_{\Gamma}=\langle\gamma v, x\rangle_{\Gamma}$, para todo $x \in V$ e $\gamma \in \Gamma$ e, portanto, $\gamma v=v$. Logo $v \in \operatorname{Fix}(\Gamma)$. Segue da hipótese que $v=0$.

Então $f(x)=\langle 0, x\rangle_{\Gamma}=0$, para todo $x \in V$, ou seja $f$ é nula.

$(c \Rightarrow b)$ Seja $g: V \rightarrow V \Gamma$ - equivariante. Defina

$$
\begin{aligned}
L: \quad V & \rightarrow \mathbb{R} \\
x & \mapsto\langle g(0), x\rangle_{\Gamma} .
\end{aligned}
$$

Note que a função $L$ é linear e invariante pela ação de $\Gamma$. Segue da hipótese que $L(x)=0$ para todo $x \in V$, em particular $L(g(0))=\langle g(0), g(0)\rangle_{\Gamma}=0$, e então $g(0)=0$.

O próximo teorema apresenta uma fórmula para o cálculo da dimensão do subespaço de ponto fixo $\operatorname{Fix}(\Sigma)$, que depende somente do traço $\operatorname{tr}\left(\rho_{\sigma}\right)$. Denotemos $\operatorname{tr}\left(\rho_{\sigma}\right)$ simplesmente por $\operatorname{tr}(\sigma)$.

Teorema 1.4.11. (Fórmula do Traço) Seja $\Gamma$ um grupo de Lie compaco agindo linearmente em $V$ e seja $\Sigma$ subgrupo de $\Gamma$. Então

$$
\operatorname{dim} \operatorname{Fix}(\Sigma)=\int_{\gamma \in \Sigma} \operatorname{tr}(\gamma)
$$

onde $\int_{\Sigma}$ denota a integral de Haar normalizada em $\Sigma$, isto é, $\int_{\Sigma} 1=1$.

Demonstração: Primeiramente defina a aplicação $A: V \rightarrow V$ dada por

$$
A(v)=\left(\int_{\Sigma} \gamma\right)(v) .
$$


Mais especificamente, para $v=\left(v_{1}, \ldots, v_{n}\right) \in V$ e $\rho_{\sigma}=\left(\gamma_{i j}\right)$, temos

$$
A(v)=\left(\begin{array}{ccc}
\int_{\Sigma} \gamma_{11} & \cdots & \int_{\Sigma} \gamma_{1 n} \\
\vdots & \ddots & \vdots \\
\int_{\Sigma} \gamma_{n 1} & \cdots & \int_{\Sigma} \gamma_{n n}
\end{array}\right)\left(\begin{array}{c}
v_{1} \\
\vdots \\
v_{n}
\end{array}\right)
$$

A aplicação $A$ é linear e $\Sigma$-invariante pois trata-se de uma integral de Haar. Agora, note que

$$
\begin{aligned}
A \circ A & =A \circ\left(\int_{\Sigma} \sigma\right) \\
& =\int_{\gamma \in \Sigma} \gamma\left(\int_{\Sigma} \sigma\right) \\
& =\int_{\gamma \in \Sigma} \int_{\sigma \in \Sigma} \gamma \sigma \\
& =\int_{\Sigma} A \\
& =A .
\end{aligned}
$$

A igualdade (1.44) ocorre pois $\int_{\Sigma} 1=1$. Logo $A^{2}=A$ e, portanto, $A$ é uma projeção linear. Então

$$
\begin{aligned}
& V=\operatorname{ker} A \oplus \operatorname{Im} A \\
& A_{\mid \operatorname{Im} A}=i d
\end{aligned}
$$

Das igualdades (1.45) e (1.46), segue que $\operatorname{ker} A$ e $\operatorname{Im} A$ são invariantes por $A$ e, portanto, $\operatorname{tr}(A)=\operatorname{dim}(\operatorname{Im} A)$. Então, basta mostrar que $\operatorname{Im} A=\operatorname{Fix}(\Sigma)$. De fato, dado $v \in \operatorname{Im} A$ temos $A(v)=v$. Então para $\sigma \in \Sigma$ temos

$$
\begin{aligned}
\sigma v & =\sigma\left(\int_{\gamma \in \Sigma} \gamma\right)(v) \\
& =\left(\int_{\gamma \in \Sigma} \sigma \gamma\right)(v) \\
& =\left(\int_{\gamma \in \Sigma} \gamma\right)(v) \\
& =A(v)=v,
\end{aligned}
$$

sendo a igualdade (1.47) proveniente da invariância da integral de Haar. Logo $\operatorname{Im} A \subset$ $\operatorname{Fix}(\Sigma)$.

Por outro lado, se $v \in \operatorname{Fix}(\Sigma)$, segue que $\gamma v=v$ para todo $\gamma \in \Sigma$, ou seja,

$$
A(v)=\int_{\Sigma} \sigma v=\int_{\Sigma} v=\left(\int_{\Sigma} I\right) v=\left(\begin{array}{ccc}
\int_{\Sigma} 1 & & 0 \\
& \ddots & \\
0 & & \int_{\Sigma} 1
\end{array}\right) v=\left(\int_{\Sigma} 1\right) I(v)=v
$$


Assim $v \in \operatorname{Im} A$ e, portanto, $\operatorname{Fix}(\Sigma) \subset \operatorname{Im} A$.

Notemos que para $\Sigma$ finito, temos

$$
\operatorname{dim}_{\operatorname{Fix}_{V}}(\Sigma)=\frac{1}{|\Sigma|} \sum_{\sigma \in \Sigma} \operatorname{tr}(\sigma)
$$

Finalmente apresentamos um resultado decorrente do Teorema 1.4.11 acima. Antes, uma definição:

Definição 1.4.12. Sejam $H_{1}, \ldots, H_{k}$ subgrupos de $\Sigma$. Dizemos que $\Sigma$ é uma união disjunta de $H_{1}, \ldots, H_{k}$ se

a) $\Sigma=H_{1} \cup \ldots \cup H_{k}$;

b) $H_{i} \cap H_{j}=\{I\}$, para todo $i \neq j$.

Denotamos essa união disjunta por $\Sigma=H_{1} \dot{\cup} \ldots \dot{\cup} H_{k}$.

Lema 1.4.13. Seja $\Gamma$ um grupo de Lie compacto agindo em $V$ e seja $\Sigma=H_{1} \dot{\cup} \ldots \dot{\cup} H_{k}$ um subgrupo finito de $\Gamma$. Então

$$
\operatorname{dim} \operatorname{Fix}(\Sigma)=\frac{1}{|\Sigma|}\left(\sum_{i=1}^{k}\left|H_{i}\right| \operatorname{dim} \operatorname{Fix}\left(H_{i}\right)-(k-1) \operatorname{dim} V\right)
$$

Demonstração: Pelo Teorema 1.4.11, temos

$$
\operatorname{dim} \operatorname{Fix}(\Sigma)=\frac{1}{|\Sigma|} \sum_{\gamma \in \Sigma} \operatorname{tr}(\sigma)=\frac{1}{|\Sigma|}\left(\sum_{i=1}^{k} \sum_{h \in H_{i}} \operatorname{tr}(h)-(k-1) \operatorname{tr}(I)\right) .
$$

$\operatorname{Mas} \operatorname{tr}(I)=\operatorname{dim} V \mathrm{e}$

$$
\operatorname{dim} \operatorname{Fix}\left(H_{i}\right)=\frac{1}{\left|H_{i}\right|} \sum_{h \in H_{i}} \operatorname{tr}(h)
$$

Reescrevendo a equação (1.48) chegamos ao resultado esperado. 



\section{Capítulo 2}

\section{A teoria reversível-equivariante}

Num sistema dinâmico podem ocorrer a presença simultânea de simetrias e antisimetrias. Quando ambas ocorrem simultaneamente o sistema é chamado de reversivelequivariante e o conjunto desses objetos tem a estrutura de grupo e denominamos por $\Gamma$. Tal fato implica na existência de um subgrupo normal de índice 2 formado pela simetrias de $\Gamma$ e denotado por $\Gamma_{+}$, e o seu complementar, o conjunto das anti-simetrias, é denotado por $\Gamma_{-}$. Vamos entender no que segue que o grupo $\Gamma_{+}$tem um papel fundamental para a obtenção dos geradores do módulo das aplicações polinomiais $\Gamma$ reversíveis-equivariantes.

A noção da presença de simetrias e anti-simetrias em equações diferenciais consiste de transformações nas variáveis de estado que preservam o retrato de fase. Enquanto simetrias levam trajetórias em trajetórias, preservando a direção com o tempo, antisimetrias levam trajetórias em trajetórias, revertendo a direção com o tempo. É claro que do ponto de vista geométrico essa reversão corresponde a uma inversão das flechas. Na figura 2.1(a) todas as rotações em torno da origem representam uma simetria, enquanto que a reflexão em relação ao eixo- $x$ é uma anti-simetria. Na figura 2.1(b) a reflexão com relação ao eixo- $x$ é uma simetria, e a rotação de ângulo $\pi / 2$ em torno da origem representa uma anti-simetria.

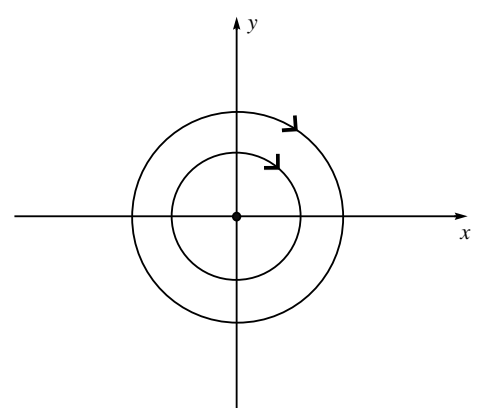

(a)

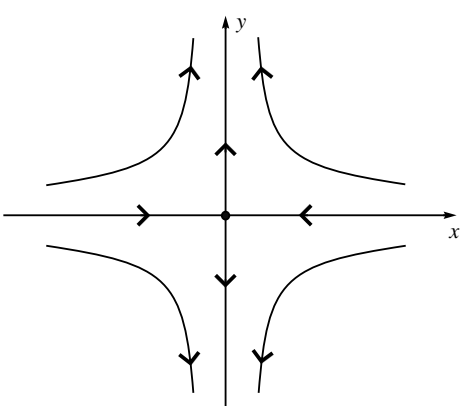

(b)

Figura 2.1: Retratos de fase de campos vetoriais reais com simetrias e anti-simetrias.

No decorrer deste capítulo assumimos $\Gamma$ um grupo de Lie compacto agindo linearmente num espaço $V$ de dimensão finita. O ponto principal deste capítulo é analisar 
as relações existentes entre a teoria de reversibilidade de um sistema dinâmico com a teoria de invariantes.

Descrevemos um algoritmo para a construção de um conjunto de geradores para o módulo dos anti-invariantes, através da teoria de invariantes. Por fim, apresentamos um outro algoritmo para determinar um conjunto de geradores para reversíveis-equivariantes em termos dos geradores dos anti-invariantes e dos $\Gamma_{+^{-}}$equivariantes.

\subsection{O contexto reversível-equivariante}

Seja $\Gamma$ um grupo de Lie compacto e considere o homomorfismo

$$
\sigma: \Gamma \rightarrow \mathbf{Z}_{2}=\{-1,1\}
$$

Note que $\sigma$ define uma representação 1-dimensional de $\Gamma$, dada por $x \mapsto \sigma_{\gamma} x$. Se $\sigma$ é não trivial, então $\Gamma_{+}=\operatorname{ker}(\sigma)$ é um subgrupo normal de $\Gamma$ de índice 2 , e o seu complementar $\Gamma_{-}$é um conjunto não trivial. Fixando um $\delta \in \Gamma_{-}$temos a decomposição de $\Gamma$ como união disjunta

$$
\Gamma=\Gamma_{+} \dot{U} \Gamma_{-}=\Gamma_{+} \dot{U} \delta \Gamma_{+} .
$$

Nesse caso o grupo $\Gamma$ é chamado de grupo de simetrias e anti-simetrias. Se $\gamma \in \Gamma_{+}$ dizemos que $\gamma$ é uma simetria em $\Gamma$, enquanto que um elemento de $\Gamma_{-}$é chamado de anti-simetria ou simetria reversível.

Note que o produto entre duas simetrias e entre duas anti-simetrias é uma simetria. Por outro lado, o produto de uma simetria com uma anti-simetria é uma anti-simetria. Observe ainda que o anel $\mathcal{P}_{V}\left(\Gamma_{+}\right)$tem estrutura de módulo sobre o anel $\mathcal{P}_{V}(\Gamma)$.

Sejam $(\rho, V)$ e $(\eta, W)$ representações de $\Gamma$ e considere um homomorfismo $\sigma: \Gamma \rightarrow$ $\mathbf{Z}_{2}=\{-1,1\}$ não trivial. Mudamos a notação $\rho_{\gamma}$ e $\eta_{\gamma}$ para $\rho(\gamma)$ e $\eta(\gamma)$ respectivamente e damos as seguintes definições:

Definição 2.1.1. Um germe de função $\tilde{f}: V \rightarrow \mathbb{R}$ é chamado de $\Gamma$-anti-invariante se

$$
\tilde{f}(\rho(\gamma)(x))=\sigma(\gamma) \tilde{f}(x), \forall \gamma \in \Gamma .
$$

Denotamos por $\mathcal{F}_{V}(\Gamma)$ o espaço dos germes de funções $\tilde{f}: V \rightarrow \mathbb{R} \Gamma$-anti-invariantes e por $\mathcal{Q}_{V}(\Gamma)$ o espaço de todas as funções polinomiais $\Gamma$-anti-invariantes, os quais tem estrutura de módulo sobre os anéis $\mathcal{E}_{V}(\Gamma)$ e $\mathcal{P}_{V}(\Gamma)$, respectivemente.

Definição 2.1.2. Um germe de aplicação $\tilde{g}: V \rightarrow W$ é chamado $\Gamma$-reversível-equivariante se

$$
\tilde{g}(\rho(\gamma) x)=\sigma(\gamma) \eta(\gamma) \tilde{g}(x), \forall \gamma \in \Gamma
$$

Denotamos por $\overrightarrow{\mathcal{F}}_{V, W}(\Gamma)$ o espaço de todos os germes de aplicação $g: V \rightarrow W$ $\Gamma$-equivariantes e por $\overrightarrow{\mathcal{Q}}_{V, W}(\Gamma)$ o espaço de todas as aplicaçõs polinomiais $\Gamma$-reversíveisequivariantes, os quais têm estrutura de módulos sobre os anéis $\mathcal{E}_{V}(\Gamma)$ e $\mathcal{P}_{V}(\Gamma)$, respectivemente.

Definição 2.1.3. A representação $\sigma$-dual de $\rho$ é a representação $\rho_{\sigma}$ de $\Gamma$ em $\boldsymbol{G L}(V)$ dada por

$$
\begin{aligned}
\rho_{\sigma}: & \Gamma \rightarrow \boldsymbol{G} \boldsymbol{L}(V) \\
\gamma & \mapsto \sigma(\gamma) \rho(\gamma) .
\end{aligned}
$$


Observe que $\left(\rho_{\sigma}\right)_{\sigma}=\rho$.

Definição 2.1.4. Dizemos que a representação $(\rho, V)$ de $\Gamma$ é $\sigma$-auto-dual se ela é $\Gamma$ isomorfa à sua representação dual $\left(\rho_{\sigma}, V\right)$, ou seja, se existe um isomorfismo linear $\Gamma$-equivariante $L:(\rho, V) \rightarrow\left(\rho_{\sigma}, V\right)$, o que equivale dizer que existe um isomorfismo linear $\Gamma$-reversivel-equivariante $L: V \rightarrow V$.

Neste caso, dizemos que $V$ é um espaço auto-dual.

Observação 2.1.5. Podemos reescrever a Definição 2.1.2 como $\tilde{g}(\rho(\gamma) x)=\eta_{\sigma}(\gamma) \tilde{g}(x)$. Neste caso observamos que um germe de aplicação $\Gamma$-reversivel-equivariante de $(\rho, V)$ em $(\eta, W)$ é um germe $\Gamma$-equivariante de $(\rho, V)$ em $\left(\eta_{\sigma}, W\right)$.

De forma análoga reescrevemos a Definição 2.1.1 como $\tilde{f}(\rho(\gamma)(x))=\sigma(\gamma) \tilde{f}(x)$, notando que uma função anti-invariante de $V$ em $\mathbb{R}$ é uma função $\Gamma$-equivariante de $(\rho, V)$ em $(\sigma, \mathbb{R})$.

Assim, o Teorema de Poénaru (Teorema 1.3.18) nos garante a existência de um conjundo finito de geradores para os módulos $\mathcal{Q}_{V}(\Gamma)$ e $\overrightarrow{\mathcal{Q}}_{V, W}(\Gamma)$. Como consequência do Teorema de Poénaru, podemos restringir nosso estudo ao caso polinomial.

\subsection{Teoria de caracteres}

Seja $(\rho, V)$ uma representação de um grupo de Lie compacto $\Gamma$ em $V$. O caracter da representação $(\rho, V)$ é uma função que associa a cada elemento de um grupo $\Gamma$ um elemento dos reais, isto é, o corpo do espaço da representação $V$, constituindo uma ferramenta importante na teoria invariante.

\subsubsection{Caracter de uma representação}

Sejam $(\rho, V)$ e $(\eta, W)$ representações de $\Gamma$.

Definição 2.2.1. O caracter $\mathcal{X}$ da representação $(\rho, V)$ é dado pela função $\mathcal{X}_{V}: \Gamma \rightarrow \mathbb{R}$,

$$
\mathcal{X}_{V}(\gamma)=\operatorname{tr}(\rho(\gamma))
$$

onde $\operatorname{tr}(\rho(\gamma))$ denota o traço da matriz $\rho(\gamma)=\rho_{\gamma}$.

Para todo $\gamma, \delta \in \Gamma$ temos

$$
\mathcal{X}_{V}\left(\gamma \delta \gamma^{-1}\right)=\mathcal{X}_{V}(\delta)
$$

implicando que $\mathcal{X}_{V}$ é constante nas classes de conjugação de $\Gamma$. Note que se $V$ é um espaço vetorial sob a ação ortogonal de $\Gamma$ então

$$
\mathcal{X}_{V}\left(\gamma^{-1}\right)=\mathcal{X}_{V}(\gamma), \forall \gamma \in \Gamma
$$

Quando $\Gamma$ age simultaneamente em $V$ e $W$ definimos o produto interno entre os caracteres $\mathcal{X}_{V}$ e $\mathcal{X}_{W}$ por

$$
\left\langle\mathcal{X}_{V}, \mathcal{X}_{W}\right\rangle=\int_{\Gamma} \mathcal{X}_{V}(\gamma) \mathcal{X}_{W}(\gamma)
$$


Lema 2.2.2. Representações Isomorfas têm os mesmos caracteres.

Demonstração: Sejam $(\rho, V)$ e $(\eta, W)$ representações $\Gamma$-isomorfas. Então existe um isomorfisvo linear $A: V \rightarrow W \Gamma$ - equivariante tal que $\rho(\gamma)=A^{-1} \eta(\gamma) A$, implicando em

$$
\mathcal{X}_{V}(\gamma)=\operatorname{tr}(\rho(\gamma))=\operatorname{tr}\left(A^{-1} \eta(\gamma) A\right)=\operatorname{tr}(\eta(\gamma))=\mathcal{X}_{W}(\gamma), \forall \gamma \in \Gamma
$$

Dando prosseguimento à teoria definimos o produto tensorial entre os espaços vetoriais $V$ e $W$.

Definição 2.2.3. O produto tensorial de $V$ e $W$ é um espaço vetorial $V \otimes W$ juntamente com uma aplicação bilinear

$$
\begin{aligned}
\tau: V \times W & \rightarrow V \otimes W \\
(x, y) & \mapsto x \otimes y,
\end{aligned}
$$

com a propriedade universal. Ou seja, dado qualquer espaço vetorial $U$ e uma aplicação bilinear $\beta: V \times W \rightarrow U$, existe uma única aplicação linear $B: V \otimes W \rightarrow U$ tal que $\beta=B \circ \tau$.

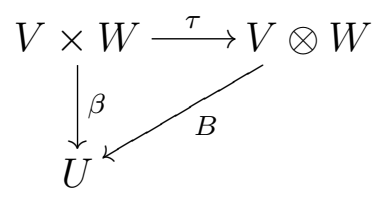

Tal propriedade determina o produto tensorial a menos de isomorfismos canônicos. Em particular o produto $n$-tensorial $V^{\otimes n}=\underbrace{V \otimes \ldots \otimes V}_{n \text { vezes }}$ é munido de uma aplicação $n$-multilinear

$$
\begin{aligned}
V \times \ldots \times V: & \rightarrow V \otimes \ldots \otimes V \\
\left(x_{1}, \ldots, x_{n}\right) & \mapsto x_{1} \otimes \ldots \otimes x_{n} .
\end{aligned}
$$

Definimos a ação do grupo das permutações de $n$ elementos $\mathbf{S}_{n}$ em $V^{\otimes n}$ por

$$
\tau\left(x_{1}, \ldots, x_{n}\right)=x_{\tau^{-1}(1)} \otimes \ldots \otimes x_{\tau^{-1}(n)} .
$$

Definição 2.2.4. Dadas duas representações $(\rho, V)$ e $(\eta, W)$ de $\Gamma$, definimos a representação de $\Gamma$ no produto tensorial $V \otimes W$ por

$$
\xi: \Gamma \rightarrow \boldsymbol{G L}(V \otimes W), \text { onde }
$$

$\xi(\gamma)(x \otimes y)=\rho(\gamma) x \otimes \eta(\gamma) y$

Dessa iteração obtemos a representação de $\Gamma$ em $V^{\otimes n}$.

Também definimos a representação de $\Gamma$ na soma direta $V \oplus W$ por

$$
\varrho: \Gamma \rightarrow \boldsymbol{G L}(V \oplus W), \text { onde }
$$

$\varrho(\gamma)(x \oplus y)=\rho(\gamma) x \oplus \eta(\gamma) y$ 
Se considerarmos a $n$-ésima potência tensorial simétrica

$$
S^{n} V=\left\{x \in V^{\otimes n}: \tau x=x \forall \tau \in \mathbf{S}_{n}\right\},
$$

então $S^{n} V$ é um subespaço $\Gamma$-invariante de $V^{\otimes n}$, uma vez que a ação do grupo de permutações $\mathbf{S}_{n}$ em $V^{\otimes n}$ comuta com a ação de $\Gamma$ em $V^{\otimes n}$.

Considere o espaço $V^{*}=\operatorname{Hom}(V, R)$ dos funcionais lineares de $V$ em $\mathbb{R}$. Então existe uma representação natural de $\Gamma$ em $V^{*}$ definida por

$$
\rho^{*}: \Gamma \rightarrow G L\left(V^{*}\right)
$$

onde, $\left[\rho^{*}(\gamma) f\right](v)=f\left(\rho\left(\gamma^{-1}\right)(v)\right.$, para todo $\gamma \in \Gamma, v \in V, f \in V^{*}$.

Finalizamos esta subseção com algumas propriedade de caracteres.

Proposição 2.2.5. Sejam $(\rho, V)$ e $(\eta, W)$ representações de um grupo de Lie compacto Г. Então:

a) $\mathcal{X}_{V \oplus W}=\mathcal{X}_{V}+\mathcal{X}_{W}$;

b) $\mathcal{X}_{V \otimes W}=\mathcal{X}_{V} \mathcal{X}_{W}$

c) $\mathcal{X}_{V^{*}}(\gamma)=\mathcal{X}_{V}\left(\gamma^{-1}\right)$, para todo $\gamma \in \Gamma$.

Demonstração: Veja Bröcker [6], II, Proposition 4.10.

\subsubsection{Séries de Hilbert-Poincaré e fórmulas de Molien}

Uma aplicação $n$-multinlinear $\beta: \underbrace{V \times \ldots \times V}_{n \text { vezes }} \rightarrow W$ é simétrica se

$$
\beta \circ \tau=\beta, \forall \tau \in \mathbf{S}_{n}
$$

Uma função $f: V \rightarrow \mathbb{R}$ é uma função polinomial homogênea de grau $d$ se existe uma função $d$-linear simétrica

$$
\widehat{f}: \underbrace{V \times \ldots \times V}_{\text {dvezes }} \rightarrow \mathbb{R}
$$

tal que $f(x)=\widehat{f}(x, \ldots, x)$ para todo $x \in V$.

$\mathrm{O}$ anel $\mathcal{P}_{V}=\mathbb{R}\left[x_{1}, \ldots, x_{n}\right]$ é uma álgebra graduada sobre $\mathbb{R}$, isto é,

$$
\mathcal{P}_{V}=\bigoplus_{d=0}^{\infty} \mathcal{P}_{V}^{d},
$$

onde $\mathcal{P}_{V}^{d}$ é o espaço das aplicações polinomiais homogêneas de grau $d$. Uma vez que a ação de $\Gamma$ em $V$ é linear, se $f \in \mathcal{P}_{V}^{d}$ para algum $d$ temos $\gamma f \in \mathcal{P}_{V}^{d}$ onde $f$ é $\Gamma$ - invariante. Portanto, o anel $\mathcal{P}_{V}(\Gamma)$ tem a estrutura de uma álgebra graduada dada por

$$
\mathcal{P}_{V}(\Gamma)=\bigoplus_{d=0}^{\infty} \mathcal{P}_{V}^{d}(\Gamma),
$$


onde $\mathcal{P}_{V}^{d}(\Gamma)=\mathcal{P}_{V}(\Gamma) \cap \mathcal{P}_{V}^{d}$.

Se $\Gamma$ também age num espaço vetorial $W$, denotamos por $\overrightarrow{\mathcal{P}}_{V, W}$ o módulo sobre $\mathcal{P}_{V}$ das aplicações polinomiais de $V$ em $W$. Se $V=W$ simplificamos a notação para $\overrightarrow{\mathcal{P}}_{V}$. Observe que $\overrightarrow{\mathcal{P}}_{V, W}$ também tem estrutura de módulo graduado sobre o anel $\overrightarrow{\mathcal{P}}_{V}$, isto é

$$
\overrightarrow{\mathcal{P}}_{V, W}=\bigoplus_{d=0}^{\infty} \overrightarrow{\mathcal{P}}_{V, W}^{d},
$$

onde $\overrightarrow{\mathcal{P}}_{V, W}^{d}$ é o espaço vetorial das aplicações polinomiais homogêneas de $V \rightarrow W$ de grau $d$. Portanto, o espaço $\overrightarrow{\mathcal{P}}_{V, W}(\Gamma)$ é um módulo graduado sob o anel $\mathcal{P}_{V}(\Gamma)$,

$$
\overrightarrow{\mathcal{P}}_{V, W}(\Gamma)=\bigoplus_{d=0}^{\infty} \overrightarrow{\mathcal{P}}_{V, W}^{d}(\Gamma),
$$

onde $\overrightarrow{\mathcal{P}}_{V, W}^{d}(\Gamma)=\overrightarrow{\mathcal{P}}_{V, W}(\Gamma) \cap \overrightarrow{\mathcal{P}}_{V, W}^{d}$.

A série de Hilbert para o anel $\mathcal{P}_{V}(\Gamma)$ é uma função geradora para a dimensão do espaço vetorial dos polinômios invariantes homogêneos de cada grau e é definida pela série formal

$$
\Phi_{V}^{\Gamma}(t)=\sum_{d=0}^{\infty} \operatorname{dim} \mathcal{P}_{V}^{d}(\Gamma) t^{d} .
$$

O seguinte teorema fornece uma fórmula explícita para a série de Hilbert acima em termos da integral de Haar normalizada sob $\Gamma$ :

Teorema 2.2.6. (Molien) Seja $(\rho, V)$ uma representação de um grupo de Lie compacto $\Gamma$ em $V$. Então a série de Hilbert-Poincaré de $\mathcal{P}_{V}(\Gamma)$ é dada por

$$
\Phi_{V}^{\Gamma}(t)=\int_{\Gamma} \frac{1}{\operatorname{det}(1-t \rho(\gamma))}
$$

Demonstração: Veja Sturmfels [22] para a prova no caso finito e Sattinger [20] para a extensão ao caso de um grupo de Lie compacto.

De forma análoga, a série de Hilbert-Poincaré de $\overrightarrow{\mathcal{P}}_{V, W}(\Gamma)$ é uma função geradora para a dimensão dos espaços vetoriais dos polinômios homogêneos equivariantes em cada grau e é definida pela série formal

$$
\Psi_{V, W}^{\Gamma}(t)=\sum_{d=0}^{\infty} \operatorname{dim} \overrightarrow{\mathcal{P}}_{V, W}^{d}(\Gamma) t^{d} .
$$

A generalização do Teorema de Molien para o caso equivariante é dado por Sattinger [20].

Teorema 2.2.7. (Molien equivariante) Sejam $(\rho, V)$ e $(\eta, W)$ representações de um grupo de Lie compacto $\Gamma$. Então a série de Hilbert para o módulo $\overrightarrow{\mathcal{P}}_{V, W}(\Gamma)$ é dada por

$$
\Psi_{V, W}(t)=\int_{\Gamma} \frac{\mathcal{X}_{W}(\gamma)}{\operatorname{det}(1-t \rho(\gamma))}
$$


Em particular, se $(\rho, V)=(\eta, W)$ então

$$
\Psi_{V}(t)=\int_{\Gamma} \frac{\mathcal{X}_{V}(\gamma)}{\operatorname{det}(1-t \rho(\gamma))}
$$

Demonstração: Veja Gatermann [14], 12, Theorem 12.2.

Agora definimos a série de Hilbert para os anti-invariantes e reversíveis-equivariantes. Começamos observando que $\mathcal{Q}_{V}(\Gamma)$ é um subespaço de $\mathcal{P}_{V}(\Gamma)$ e $\overrightarrow{\mathcal{Q}}_{V, W}(\Gamma)$ é um subespaço de $\overrightarrow{\mathcal{P}}_{V, W}(\Gamma)$, e sendo assim ambos têm estrutura de módulos graduados sobre o anel $\mathcal{P}_{V}(\Gamma)$, ou seja,

$$
\mathcal{Q}_{V}(\Gamma)=\bigoplus_{d=0}^{\infty} \mathcal{Q}_{V}^{d}(\Gamma)
$$

e

$$
\overrightarrow{\mathcal{Q}}_{V, W}(\Gamma)=\bigoplus_{d=0}^{\infty} \overrightarrow{\mathcal{Q}}_{V, W}^{d}(\Gamma),
$$

onde $\mathcal{Q}_{V}^{d}(\Gamma)=\mathcal{Q}_{V}(\Gamma) \cap \mathcal{P}_{V}^{d}$ e $\overrightarrow{\mathcal{Q}}_{V, W}^{d}(\Gamma)=\overrightarrow{\mathcal{Q}}_{V, W}(\Gamma) \cap \overrightarrow{\mathcal{P}}_{V, W}^{d}$.

As séries de Hilbert para os módulos $\mathcal{Q}_{V}(\Gamma)$ e $\overrightarrow{\mathcal{Q}}_{V, W}(\Gamma)$ podem ser definidas da mesma forma que definimos para $\mathcal{P}_{V}(\Gamma)$ e $\overrightarrow{\mathcal{P}}_{V, W}(\Gamma)$, dadas por

$$
\tilde{\Phi}_{V}^{\Gamma}(t)=\sum_{d=0}^{\infty} \operatorname{dim} \mathcal{Q}_{V}^{d}(\Gamma) t^{d}
$$

e

$$
\tilde{\Psi}_{V}^{\Gamma}(t)=\sum_{d=0}^{\infty} \operatorname{dim} \overrightarrow{\mathcal{Q}}_{V, W}^{d}(\Gamma) t^{d} .
$$

Como sabemos os casos anti-invariante e reversível-equivariante se reduzem ao caso equivariante. De fato, considere o homomorfismo $\sigma: \Gamma \rightarrow\{-1,1\}$ definido em (2.1) e sejam $(\rho, V)$ e $(\sigma, \mathbb{R})$ representações de $\Gamma$ em $V$ e $\mathbb{R}$ respectivamente. Então se $f: V \rightarrow \mathbb{R}$ é um polinômio $\Gamma$-anti-invariante $\left(f \in \mathcal{Q}_{V}(\Gamma)\right)$, temos $f \in \overrightarrow{\mathcal{P}}_{V, \mathbb{R}}(\Gamma)$. Se considerarmos a representação dual, $\left(\eta_{\sigma}, W\right)$ de $\Gamma$ em $W$, então $g: V \rightarrow W$ é $\Gamma$-reversível-equivariante $\left(g \in \overrightarrow{\mathcal{Q}}_{V, W}(\Gamma)\right)$ se $g \in \overrightarrow{\mathcal{P}}_{V, W}(\Gamma)$.

Então do Teorema de Molien 2.2.7 para o caso equivariante, obtemos as fórmulas de Molien para as Séries de Hilbert dos módulos $\mathcal{Q}_{V}(\Gamma)$ e $\overrightarrow{\mathcal{Q}}_{V, W}(\Gamma)$ :

$$
\tilde{\Phi}_{V}^{\Gamma}(t)=\int_{\Gamma} \frac{\sigma(\gamma)}{\operatorname{det}(1-t \rho(\gamma))}
$$

e

$$
\tilde{\Psi}_{V, W}^{\Gamma}(t)=\int_{\Gamma} \frac{\sigma(\gamma) \mathcal{X}_{W}(\gamma)}{\operatorname{det}(1-t \rho(\gamma))}
$$

De fato, observe que para o caso anti-invariante temos

$$
\tilde{\Phi}_{V}^{\Gamma}(t)=\int_{\Gamma} \frac{\mathcal{X}_{\mathbb{R}}(\gamma)}{\operatorname{det}(1-t \rho(\gamma))}=\int_{\Gamma} \frac{\operatorname{tr}(\sigma(\gamma))}{\operatorname{det}(1-t \rho(\gamma))}=\int_{\Gamma} \frac{\sigma(\gamma)}{\operatorname{det}(1-t \rho(\gamma))},
$$


e para o caso reversível-equivariante temos

$$
\begin{gathered}
\tilde{\Psi}_{V, W}^{\Gamma}(t)=\int_{\Gamma} \frac{\mathcal{X}_{W}(\gamma)}{\operatorname{det}(1-t \rho(\gamma))}=\int_{\Gamma} \frac{\operatorname{tr}\left(\eta_{\sigma}(\gamma)\right)}{\operatorname{det}(1-t \rho(\gamma))}=\int_{\Gamma} \frac{\operatorname{tr}(\sigma(\gamma) \eta(\gamma))}{\operatorname{det}(1-t \rho(\gamma))}= \\
\int_{\Gamma} \frac{\sigma(\gamma) \operatorname{tr}(\eta(\gamma))}{\operatorname{det}(1-t \rho(\gamma))}=\int_{\Gamma} \frac{\sigma(\gamma) \mathcal{X}_{W}(\gamma)}{\operatorname{det}(1-t \rho(\gamma))} .
\end{gathered}
$$

\subsubsection{Fórmulas de caracter}

Sejam $V$ e $W$ espaços vetoriais de dimensão finita.

Denotamos por $\mathcal{L}_{S}^{d}(V)$ o espaço de todas as funções $d$-multilineares simétricas de $V^{d}$ em $W$. Segue que a aplicação

$$
\begin{array}{cl}
\mathcal{L}_{S}^{d}(V) & \rightarrow \mathcal{P}_{V}^{d} \\
f & \mapsto f
\end{array}
$$

é um isomorfismo.

De forma análoga denotamos por $\mathcal{L}_{S}^{d}(V, W)$ o espaço de todas as aplicações $d$-multilineares simétricas de $V^{d}$ a valores em $W$. Então a aplicação

$$
\begin{aligned}
\mathcal{L}_{S}^{d}(V, W) & \rightarrow \overrightarrow{\mathcal{P}}_{V, W}^{d} \\
\hat{f} & \mapsto f
\end{aligned}
$$

é um isomorfismo, que se reduz ao isomorfismo (2.14) quando $W=\mathbb{R}$.

Agora considere a d-ésima potência tensorial simétrica $S^{d}(V)$. Podemos caracterizála da seguinte forma:

Proposição 2.2.8. Existe um isomorfismo canônico

$$
\operatorname{Hom}\left(S^{d} V, W\right) \rightarrow \mathcal{L}_{S}^{d}(V, W) .
$$

Demonstração: Veja Goodman [16], página 621.

O seguinte teorema usa a teoria de caracter para a obtenção da dimensão de $\overrightarrow{\mathcal{P}}_{V W}^{d}(\Gamma)$.

Teorema 2.2.9. (Sattinger) Sejam $(\rho, V)$ e $(\eta, W)$ representações de um grupo de Lie compacto $\Gamma$, com caracteres correspondentes $\mathcal{X}_{V}$ e $\mathcal{X}_{W}$. Então

$$
\operatorname{dim} \overrightarrow{\mathcal{P}}_{V, W}^{d}(\Gamma)=\int_{\Gamma} \mathcal{X}_{d}\left(\gamma^{-1}\right) \mathcal{X}_{W}(\gamma)
$$

onde $\mathcal{X}_{d}$ representa o caracter da ação induzida de $\Gamma$ em $S^{d}(V)$.

Demonstração: Veja Baptistelli [4], capítulo 3, Teorema 3.1.3.

Como a representação de $\Gamma$ em $V$ é ortogonal e $V$ é real, temos $\mathcal{X}_{V}\left(\gamma^{-1}\right)=\mathcal{X}_{V}(\gamma)$. Em particular,

$$
\operatorname{dim} \overrightarrow{\mathcal{P}}_{V W}^{d}(\Gamma)=\int_{\Gamma} \mathcal{X}_{d}(\gamma) \mathcal{X}_{W}(\gamma)
$$

Segue diretamente desse teorema o seguinte corolário: 
Corolário 2.2.10. Considere as hipóteses do teorema acima. Então

$$
\begin{gathered}
\operatorname{dim} \mathcal{P}_{V}^{d}(\Gamma)=\int_{\Gamma} \mathcal{X}_{d}(\gamma), \\
\operatorname{dim} \mathcal{Q}_{V}^{d}(\Gamma)=\int_{\Gamma} \sigma(\gamma) \mathcal{X}_{d}(\gamma)
\end{gathered}
$$

$e$

$$
\operatorname{dim} \overrightarrow{\mathcal{Q}}_{V, W}^{d}(\Gamma)=\int_{\Gamma} \sigma(\gamma) \mathcal{X}_{d}(\gamma) \mathcal{X}_{W}(\gamma)
$$

onde $\mathcal{X}_{d}$ representa o caracter da ação induzida de $\Gamma$ em $S^{d}(V)$.

Demonstração: Segue direto do teorema anterior considerando $W=\mathbb{R}$ sob a ação trivial de $\Gamma$ para o caso invariante, tomando $(\eta, W)=(\sigma, \mathbb{R})$ para o caso anti-invariante, e $(\eta, W)=\left(\rho_{\sigma}, V\right)$ para o caso reversível-equivariante.

Existe uma fórmula recursiva para o cálculo do caracter $\mathcal{X}_{d}$ correspondente à ação de $\Gamma$ em $S^{d} V$, dada por

$$
d \mathcal{X}_{d}(\gamma)=\sum_{i=0}^{d-1} \mathcal{X}\left(\gamma^{d-i}\right) \mathcal{X}_{i}(\gamma)
$$

onde $\mathcal{X}_{0}(\gamma)=1$ para todo $\gamma \in \Gamma$. Para a prova, veja Antoneli [2], Section 4 .

O seguinte teorema apresenta uma integração sobre um grupo de Lie compacto dada por iteração de integrais:

Teorema 2.2.11. (Fubini) Seja $\Gamma$ um grupo de Lie compacto e $\Sigma$ um subgrupo fechado de $\Gamma$. Para qualquer função contínua $f: \Gamma \rightarrow \mathbb{R}$ tem-se

$$
\int_{\Gamma} f(\gamma) d \gamma=\int_{\Gamma / \Sigma}\left(\int_{\Sigma} f(\gamma) d \sigma\right) d(\gamma \Sigma)
$$

onde $d(\gamma \Sigma)$ denota a medida de Haar normalizada invariante à esquerda sobre $\Gamma / \Sigma$.

Demonstração: Veja Bröcker, [6], I, Proposition 5.16.

Corolário 2.2.12. Seja $\Gamma$ um grupo de Lie compacto $e \Gamma_{+} \subset \Gamma$ um subgrupo de índice 2 em $\Gamma$. Para qualquer $f: \Gamma \rightarrow \mathbb{R}$ contínua temos

$$
\int_{\Gamma} f(\gamma)=\frac{1}{2}\left[\int_{\Gamma_{+}} f(\gamma)+\int_{\Gamma_{+}} f(\delta \gamma)\right]
$$

para $\delta \in \Gamma_{-}$fixo.

Demonstração: Como $\Gamma / \Gamma_{+}$é grupo finito com $\left[\Gamma: \Gamma_{+}\right]=2$, então a integral de Haar fica

$$
\int_{\Gamma / \Gamma_{+}}\left(\int_{\Gamma_{+}} f(\gamma)\right)=\frac{1}{2} \sum_{\gamma \in \Gamma} \int_{\Gamma_{+}} f(\gamma)=\frac{1}{2}\left[\int_{\Gamma_{+}} f(\gamma)+\int_{\Gamma_{+}} f(\delta \gamma)\right],
$$

para $\delta \in \Gamma_{-}$fixo implicando na equação (2.16).

Agora, para cada $d \in \mathbb{N}$ existe uma relação entre $\operatorname{dim} \mathcal{P}_{V}^{d}(\Gamma)$ e $\operatorname{dim} \mathcal{Q}^{d}(\Gamma)$ e entre $\operatorname{dim} \overrightarrow{\mathcal{P}}_{V, W}^{d}$ e $\operatorname{dim} \overrightarrow{\mathcal{Q}}_{V W}^{d}(\Gamma)$ dada na proposição a seguir: 
Proposição 2.2.13. Considere representações $(\rho, V)$ e $(\eta, W)$ de um grupo de Lie compacto $\Gamma$ em $V$ e $W$ respectivamente. Então

$$
\operatorname{dim} \mathcal{P}_{V}^{d}\left(\Gamma_{+}\right)=\operatorname{dim} \mathcal{P}_{V}^{d}(\Gamma)+\operatorname{dim} \mathcal{Q}_{V}^{d}(\Gamma)
$$

$e$

$$
\operatorname{dim} \overrightarrow{\mathcal{P}}_{V, W}^{d}\left(\Gamma_{+}\right)=\operatorname{dim} \overrightarrow{\mathcal{P}}_{V, W}^{d}(\Gamma)+\operatorname{dim} \overrightarrow{\mathcal{Q}}_{V, W}^{d}(\Gamma)
$$

Demonstração: Segue do Teorema 2.2.9 e dos Corolários 2.2.10 e 2.2.12 as seguintes igualdades:

$$
\begin{gathered}
\operatorname{dim} \mathcal{P}_{V}^{d}(\Gamma)=\int_{\Gamma} \mathcal{X}_{d}(\gamma)=\frac{1}{2}\left[\int_{\Gamma_{+}} \mathcal{X}_{d}(\gamma)+\int_{\Gamma_{+}} \mathcal{X}_{d}(\delta \gamma)\right] \\
\operatorname{dim} \mathcal{Q}_{V}^{d}(\Gamma)=\int_{\Gamma} \sigma(\gamma) \mathcal{X}_{d}(\gamma)=\frac{1}{2}\left[\int_{\Gamma_{+}} \mathcal{X}_{d}(\gamma)-\int_{\Gamma_{+}} \mathcal{X}_{d}(\delta \gamma)\right] \\
\operatorname{dim} \overrightarrow{\mathcal{P}}_{V, W}^{d}(\Gamma)=\int_{\Gamma} \mathcal{X}_{d}(\gamma) \mathcal{X}_{W}(\gamma)=\frac{1}{2}\left[\int_{\Gamma_{+}} \mathcal{X}_{d}(\gamma) \mathcal{X}_{W}(\gamma)+\int_{\Gamma_{+}} \mathcal{X}_{d}(\delta \gamma) \mathcal{X}_{W}(\delta \gamma)\right]
\end{gathered}
$$

e

$$
\operatorname{dim} \overrightarrow{\mathcal{Q}}_{V, W}^{d}(\Gamma)=\int_{\Gamma} \sigma(\gamma) \mathcal{X}_{d}(\gamma) \mathcal{X}_{W}(\gamma)=\frac{1}{2}\left[\int_{\Gamma_{+}} \mathcal{X}_{d}(\gamma) \mathcal{X}_{W}(\gamma)-\int_{\Gamma_{+}} \mathcal{X}_{d}(\delta \gamma) \mathcal{X}_{W}(\delta \gamma)\right]
$$

Logo,

$$
\operatorname{dim} \mathcal{P}_{V}^{d}(\Gamma)+\operatorname{dim} Q_{V}^{d}(\Gamma)=\int_{\Gamma_{+}} \mathcal{X}_{d}(\gamma)=\operatorname{dim} \mathcal{P}_{V}^{d}\left(\Gamma_{+}\right)
$$

$\mathrm{e}$

$$
\operatorname{dim} \overrightarrow{\mathcal{P}}_{V, W}^{d}(\Gamma)+\operatorname{dim} \overrightarrow{\mathcal{Q}}_{V, W}^{d}(\Gamma)=\int_{\Gamma_{+}} \mathcal{X}_{d}(\gamma) \mathcal{X}_{W}(\gamma)=\operatorname{dim} \overrightarrow{\mathcal{P}}_{V, W}^{d}\left(\Gamma_{+}\right)
$$

Agora, determinamos uma condição suficiente para que uma representação $(\rho, V)$ de um grupo de Lie compacto $\Gamma$ seja não auto-dual.

Lema 2.2.14. Seja $(\rho, V)$ uma representação auto-dual de um grupo de Lie compacto Г. Então toda anti-simetria de $\Gamma$ tem traço nulo.

Demonstração: A representação $(\rho, V)$ é $\Gamma$-isomorfa a sua representação dual $\left(\rho_{\sigma}, V\right)$. Do Lema 2.2.2 segue que $\mathcal{X}_{V}(\gamma)=\mathcal{X}_{V_{\sigma}}(\gamma)$ para todo $\gamma \in \Gamma$. Então,

$$
\operatorname{tr}(\rho(\gamma))=\mathcal{X}_{V}(\gamma)=\mathcal{X}_{V_{\sigma}}(\gamma)=\operatorname{tr}\left(\rho_{\sigma}(\gamma)\right)=\operatorname{tr}(\sigma(\gamma) \rho(\gamma))=\sigma(\gamma) \operatorname{tr}(\rho(\gamma))
$$

Se $\gamma$ é anti-simetria temos $\sigma(\gamma)=-1$, e portanto $\operatorname{tr}(\rho(\gamma))=-\operatorname{tr}(\rho(\gamma))$, implicando em $\operatorname{tr}(\rho(\gamma))=0$.

Teorema 2.2.15. Seja $(\rho, V)$ uma representação auto-dual de um grupo de Lie compacto $\Gamma$. Então as séries de Hilbert de $\overrightarrow{\mathcal{P}}_{V}(\Gamma)$ e $\overrightarrow{\mathcal{Q}}_{V}(\Gamma)$ são iguais. Além disso todo coeficiente da série de Hilbert de $\overrightarrow{\mathcal{P}}_{V}\left(\Gamma_{+}\right)$é par. 
Demonstração: Segue do Lema anterior que para toda anti-simetria $\gamma \in \Gamma_{-}$temos $\mathcal{X}_{V}(\gamma)=0$. Então

$$
\begin{gathered}
\operatorname{dim} \overrightarrow{\mathcal{P}}_{V}^{d}(\Gamma)=\int_{\Gamma} \mathcal{X}_{d}\left(\gamma^{-1}\right) \mathcal{X}_{V}(\gamma)=\int_{\Gamma_{+}} \mathcal{X}_{d}\left(\gamma^{-1}\right) \mathcal{X}_{V}(\gamma) \\
=\int_{\Gamma_{+}} \sigma(\gamma) \mathcal{X}_{d}\left(\gamma^{-1}\right) \mathcal{X}_{V}(\gamma)=\int_{\Gamma} \sigma(\gamma) \mathcal{X}_{d}\left(\gamma^{-1}\right) \mathcal{X}_{V}(\gamma)=\operatorname{dim} \overrightarrow{\mathcal{Q}}_{V}^{d}(\Gamma)
\end{gathered}
$$

Logo,

$$
\Psi_{V}^{\Gamma}(t)=\sum_{d=0}^{\infty} \operatorname{dim} \overrightarrow{\mathcal{P}}_{V}^{d}(\Gamma) t^{d}=\sum_{d=0}^{\infty} \operatorname{dim} \overrightarrow{\mathcal{Q}}_{V}^{d}(\Gamma) t^{d}=\tilde{\Psi}_{V}^{\Gamma}(t) .
$$

Portanto as séries de Hilbert de $\overrightarrow{\mathcal{P}}_{V}(\Gamma)$ e $\overrightarrow{\mathcal{Q}}_{V}(\Gamma)$ são iguais. Além disso, da Proposição 2.2.13 segue que

$$
\operatorname{dim} \overrightarrow{\mathcal{P}}_{V}^{d}\left(\Gamma_{+}\right)=\operatorname{dim} \overrightarrow{\mathcal{P}}_{V}^{d}(\Gamma)+\operatorname{dim} \overrightarrow{\mathcal{Q}}_{V}^{d}(\Gamma)=2 \operatorname{dim} \overrightarrow{\mathcal{P}}_{V}^{d}(\Gamma)
$$

mostrando que todo coeficiente da série de Hilbert de $\overrightarrow{\mathcal{P}}_{V}\left(\Gamma_{+}\right)$é par.

Observação 2.2.16. Existe um resultado análogo para as séries de Hilbert de $\mathcal{P}_{V}(\Gamma)$ e $\mathcal{Q}_{V}(\Gamma)$. Isto é, temos $\mathcal{X}_{d}(\gamma)=0$ para toda anti-simetria $\gamma \in \Gamma_{-}$, e para todo $d \in \mathbb{N}$ impar segue do Corolário 2.2.10 que $\operatorname{dim} \mathcal{P}_{V}^{d}(\Gamma)=\operatorname{dim} \mathcal{Q}_{V}^{d}(\Gamma)$ implicando em $\operatorname{dim} \mathcal{P}_{V}^{d}\left(\Gamma_{+}\right)=$ $2 \operatorname{dim} \mathcal{P}_{V}^{d}(\Gamma)$.

Exemplo 2.2.17. Considere a ação padrão do grupo simétrico $\Gamma=\mathbf{S}_{2}$ no plano gerada por

$$
\delta=\left(\begin{array}{ll}
0 & 1 \\
1 & 0
\end{array}\right) .
$$

Tomando $\delta \in \Gamma_{-}$, temos $\Gamma_{+}=\{I\}$ e portanto $\{x+y, x y\}$ e $\{x, y\}$ são bases de Hilbert para os anéis $\mathcal{P}\left(\mathbf{S}_{2}\right)$ e $\mathcal{P}\left(\Gamma_{+}\right)$respectivamente. Da Proposição 2.2.13 temos

$$
\operatorname{dim} \mathcal{Q}_{V}^{d}(\Gamma)=\operatorname{dim} \mathcal{P}_{V}^{d}\left(\Gamma_{+}\right)-\operatorname{dim} \mathcal{P}_{V}^{d}(\Gamma)
$$

Então

$$
\begin{gathered}
\operatorname{dim} \mathcal{Q}^{1}\left(\mathbf{S}_{2}\right)=2-1=1, \operatorname{dim} \mathcal{Q}^{2}\left(\mathbf{S}_{2}\right)=3-2=1 \\
\operatorname{dim} \mathcal{Q}^{3}\left(\mathbf{S}_{2}\right)=4-2=2 \mathrm{e} \operatorname{dim} \mathcal{Q}^{4}\left(\mathbf{S}_{2}\right)=5-3=2
\end{gathered}
$$

\subsection{Algoritmos}

\subsubsection{Operadores de Reynolds}

Os operadores de Reynolds constituem uma importante ferramenta para a teoria de invariantes. Seja $\Sigma$ um subgrupo fechado de $\Gamma$ e $(\rho, V)$ e $(\eta, W)$ representações de $\Gamma$. Definimos os operadores de Reynolds de $\Sigma$ para $\Gamma$ em $\mathcal{P}_{V}(\Sigma)$ e em $\overrightarrow{\mathcal{P}}_{V, W}(\Sigma)$ por

$$
R_{\Sigma}^{\Gamma}: \mathcal{P}_{V}(\Sigma) \rightarrow \mathcal{P}_{V}(\Sigma) \quad \text { e } \quad \vec{R}_{\Sigma}^{\Gamma}: \overrightarrow{\mathcal{P}}_{V, W}(\Sigma) \rightarrow \overrightarrow{\mathcal{P}}_{V, W}(\Sigma)
$$


dados por

$$
R_{\Sigma}^{\Gamma}(f)(x)=\int_{\Gamma / \Sigma} f(\gamma x) \quad \text { e } \quad \vec{R}_{\Sigma}^{\Gamma}(g)(x)=\int_{\Gamma / \Sigma} \gamma^{-1} \odot g(\gamma \cdot x)
$$

observando que · denota a ação de $\Gamma$ em $V$ e $\odot$ denota a ação de $\Gamma$ em $W$. Para nosso estudo vamos simplificar a escrita para

$$
\int_{\Gamma / \Sigma} \gamma^{-1} g(\gamma x)
$$

Quando o índice de $\Sigma$ em $\Gamma$ é um número $n$, isto é, $[\Gamma: \Sigma]=n$, então as fórmulas dos operadores de Reynolds se tornam

$$
R_{\Sigma}^{\Gamma}(f)(x)=\frac{1}{n} \sum_{\gamma \Sigma} f(\gamma x) \quad \text { e } \quad \vec{R}_{\Sigma}^{\Gamma}(g)(x)=\frac{1}{n} \sum_{\gamma \Sigma} \gamma^{-1} g(\gamma x) .
$$

Para o nosso caso particular, temos $\left[\Gamma: \Gamma_{+}\right]=2$, logo os operadores de Reynolds são dados por

$$
\begin{gathered}
R_{\Gamma_{+}}^{\Gamma}(f)(x)=\frac{1}{2} \sum_{\gamma \Gamma_{+}} f(\gamma x)=\frac{1}{2}(f(x)+f(\delta x)), \\
\vec{R}_{\Gamma_{+}}^{\Gamma_{+}}(g)(x)=\frac{1}{2} \sum_{\gamma \Gamma_{+}} \delta^{-1} f(\gamma x)=\frac{1}{2}\left(g(x)+\delta^{-1} g(\delta x)\right) .
\end{gathered}
$$

Fixando um $\delta \in \Gamma_{-}$definimos os $\sigma$-operadores de Reynolds $S_{\Gamma_{+}}^{\Gamma}: \mathcal{P}_{V}\left(\Gamma_{+}\right) \rightarrow \mathcal{P}_{V}\left(\Gamma_{+}\right)$ e $\vec{S}_{\Gamma_{+}}^{\Gamma}: \overrightarrow{\mathcal{P}}_{V, W}\left(\Gamma_{+}\right) \rightarrow \overrightarrow{\mathcal{P}}_{V, W}\left(\Gamma_{+}\right)$como

$$
\begin{gathered}
S_{\Gamma_{+}}^{\Gamma}(f)(x)=\frac{1}{2} \sum_{\gamma \Gamma_{+}} \sigma(\gamma) f(\gamma x)=\frac{1}{2}(f(x)-f(\delta x)), \\
\vec{S}_{\Gamma_{+}}^{\Gamma}(g)(x)=\frac{1}{2} \sum_{\gamma \Gamma_{+}} \sigma(\gamma) \delta^{-1} g(\gamma x)=\frac{1}{2}\left(g(x)-\delta^{-1} g(\delta x)\right) .
\end{gathered}
$$

Lema 2.3.1. Para qualquer $\delta \in \Gamma_{-}$fixado, temos

$$
\mathcal{P}_{V}(\Gamma)=\left\{f \in \mathcal{P}_{V}\left(\Gamma_{+}\right): f(\delta x)=f(x), \forall x \in V\right\}
$$

$e$

$$
\mathcal{Q}_{V}(\Gamma)=\left\{f \in \mathcal{P}_{V}\left(\Gamma_{+}\right): f(\delta x)=-f(x), \forall x \in V\right\} .
$$

Demonstração: Note que $\mathcal{P}_{V}(\Gamma) \subset \mathcal{P}_{V}\left(\Gamma_{+}\right)$pois $\Gamma_{+} \subset \Gamma$. Para todo $\gamma \in \Gamma$ temos ou $\gamma \in \Gamma_{+}$ou $\gamma=\delta \tilde{\gamma}$ para algum $\tilde{\gamma} \in \Gamma_{+}$, ou seja, $\Gamma=\Gamma_{+} \dot{U} \delta \Gamma_{+}$, com $\delta \Gamma_{+}=\Gamma_{-}$.

As inclusões " $\subseteq$ " são consequências diretas das definições de $\mathcal{P}_{V}(\Gamma)$ e $\mathcal{Q}_{V}(\Gamma)$.

Seja $f \in \mathcal{P}_{V}\left(\Gamma_{+}\right)$tal que $f(\delta x)=f(x)$. Se $\gamma \in \Gamma_{+}$então $f(\gamma x)=f(x)$ pois $f \in \mathcal{P}_{V}\left(\Gamma_{+}\right)$. Se $\gamma \in \delta \Gamma_{+}$temos

$$
f(\gamma x)=f(\delta \tilde{\gamma} x)=f(\tilde{\gamma} x)=f(x), \forall x \in V,
$$


mostrando que $f \in \mathcal{P}_{V}(\Gamma)$.

Se $f \in \mathcal{P}_{V}\left(\Gamma_{+}\right)$tal que $f(\delta x)=-f(x)$, temos $f(\gamma x)=f(x)$ para $\gamma \in \Gamma_{+}$pois $f \in \mathcal{P}_{V}\left(\Gamma_{+}\right)$. Caso contrário, se $\gamma \in \Gamma_{-}$temos

$$
f(\gamma x)=f(\delta \tilde{\gamma} x)=-f(\tilde{\gamma} x)=-f(x)=\sigma(\gamma) f(x), \forall x \in V,
$$

e portanto $f \in \mathcal{Q}_{V}(\Gamma)$.

Com isso provamos as inclusões " $\supseteq$ " e concluímos as igualdades em (2.19) e (2.20).

Denotemos por $I_{\mathcal{P}_{V}\left(\Gamma_{+}\right)}$a aplicação identidade de $\mathcal{P}_{V}\left(\Gamma_{+}\right)$.

Proposição 2.3.2. Os operadores de Reynolds $R_{\Gamma_{+}}^{\Gamma}$ e $S_{\Gamma_{+}}^{\Gamma}$ satisfazem as seguintes propriedades:

a) São homomorfismos entre $\mathcal{P}_{V}(\Gamma)$-módulos e

$$
R_{\Gamma_{+}}^{\Gamma}+S_{\Gamma_{+}}^{\Gamma}=I_{\mathcal{P}_{V}\left(\Gamma_{+}\right)} \cdot
$$

b) São projeções idempotentes com

$$
\begin{aligned}
& \operatorname{ker} R_{\Gamma_{+}}^{\Gamma}=\mathcal{Q}_{V}(\Gamma) \quad e \quad \operatorname{ker} S_{\Gamma_{+}}^{\Gamma}=\mathcal{P}_{V}(\Gamma), \\
& \operatorname{Im} R_{\Gamma_{+}}^{\Gamma}=\mathcal{P}_{V}(\Gamma) \quad e \quad \operatorname{Im} S_{\Gamma_{+}}^{\Gamma}=\mathcal{Q}_{V}(\Gamma) .
\end{aligned}
$$

c) $O$ anel $\mathcal{P}_{V}\left(\Gamma_{+}\right)$se escreve como uma soma direta de $\mathcal{P}_{V}(\Gamma)$-módulos:

$$
\mathcal{P}_{V}\left(\Gamma_{+}\right)=\operatorname{ker} R_{\Gamma_{+}}^{\Gamma} \oplus \operatorname{Im} R_{\Gamma_{+}}^{\Gamma}=\operatorname{ker} S_{\Gamma_{+}}^{\Gamma} \oplus \operatorname{Im} S_{\Gamma_{+}}^{\Gamma} .
$$

Demonstração:

a) Seja $f \in \mathcal{P}_{V}\left(\Gamma_{+}\right)$e $p \in \mathcal{P}_{V}(\Gamma)$. Para todo $x \in V$ note que

$$
R_{\Gamma_{+}}^{\Gamma}(p f)(x)=p(x) R_{\Gamma_{+}}^{\Gamma}(f)(x),
$$

e

$$
R_{\Gamma_{+}}^{\Gamma}(f+g)(x)=R_{\Gamma_{+}}^{\Gamma}(f)(x)+R_{\Gamma_{+}}^{\Gamma}(g)(x) .
$$

Então o operador $R_{\Gamma_{+}}^{\Gamma}$ é um homomorfismo entre $\mathcal{P}_{V}(\Gamma)$-módulos. De forma análoga mostramos para o $\sigma$-operador $S_{\Gamma_{+}}^{\Gamma}$.

A igualdade (2.21) segue direto das definições de $R_{\Gamma_{+}}^{\Gamma}$ e $S_{\Gamma_{+}}^{\Gamma}$.

b) Observe que para qualquer $f \in \mathcal{P}_{V}\left(\Gamma_{+}\right)$temos

$$
R_{\Gamma_{+}}^{\Gamma}(f)(\delta x)=\frac{1}{2}\left(f(\delta x)+f\left(\delta^{2} x\right)\right)=\frac{1}{2}(f(\delta x)+f(x))=R_{\Gamma_{+}}^{\Gamma}(f)(x),
$$

$\mathrm{e}$

$$
S_{\Gamma_{+}}^{\Gamma}(f)(\delta x)=\frac{1}{2}\left(f(\delta x)-f\left(\delta^{2} x\right)\right)=\frac{1}{2}(f(\delta x)-f(x))=-S_{\Gamma_{+}}^{\Gamma}(f)(x),
$$

Pelo Lema 2.3.1 segue que 


$$
R_{\Gamma_{+}}^{\Gamma}(f) \in \mathcal{P}_{V}(\Gamma) \quad \text { e } \quad S_{\Gamma_{+}}^{\Gamma}(f) \in \mathcal{Q}_{V}(\Gamma)
$$

e portanto

$$
\operatorname{Im} R_{\Gamma_{+}}^{\Gamma} \subset \mathcal{P}_{V}(\Gamma) \quad \text { e } \quad \operatorname{Im} S_{\Gamma_{+}}^{\Gamma} \subset \mathcal{Q}_{V}(\Gamma) .
$$

Por outro lado, se $f \in \mathcal{P}_{V}(\Gamma)$ então $f(\delta x)=f(x)$. Podemos escrever $f$ na forma

$$
f(x)=\frac{1}{2}(f(x)+f(\delta x))=R_{\Gamma_{+}}^{\Gamma}(f)(x) .
$$

Se $f \in \mathcal{Q}_{V}(\Gamma)$ temos $f(\delta x)=-f(x)$, e então

$$
f(x)=\frac{1}{2}(g(x)-f(\delta x))=S_{\Gamma_{+}}^{\Gamma}(f)(x) .
$$

Portanto

$$
\mathcal{P}_{V}(\Gamma) \subset \operatorname{Im} R_{\Gamma_{+}}^{\Gamma} \quad \text { e } \quad \mathcal{Q}_{V}(\Gamma) \subset \operatorname{Im} S_{\Gamma_{+}}^{\Gamma} .
$$

De $(2.22)$ e (2.23) segue que

$$
\mathcal{P}_{V}(\Gamma)=\operatorname{Im} R_{\Gamma_{+}}^{\Gamma} \quad \text { e } \quad \mathcal{Q}_{V}(\Gamma)=\operatorname{Im} S_{\Gamma_{+}}^{\Gamma} .
$$

Agora seja $f \in \operatorname{ker} R_{\Gamma_{+}}^{\Gamma} \subset \mathcal{P}_{V}\left(\Gamma_{+}\right)$e mostremos que $f(\delta x)=-f(x)$. Temos

$$
R_{\Gamma_{+}}^{\Gamma}(f)(x)=\frac{1}{2}(f(x)+f(\delta x))=0,
$$

e portanto $f(\delta x)=-f(x)$ para todo $x \in V$, mostrando que $f \in \mathcal{Q}_{V}(\Gamma)$. Por outro lado, se $f \in \mathcal{Q}_{V}(\Gamma)$ então $f(\delta x)=-f(x)$ e então $R_{\Gamma_{+}}^{\Gamma}(f)(x)=0$ para todo $x \in V$, implicando que $\mathcal{Q}_{V}(\Gamma) \subset \operatorname{ker} R_{\Gamma_{+}}^{\Gamma}$. Logo

$$
\mathcal{Q}_{V}(\Gamma)=\operatorname{ker} R_{\Gamma_{+}}^{\Gamma} .
$$

Se $f \in \operatorname{ker} S_{\Gamma_{+}}^{\Gamma} \subset \mathcal{P}_{V}\left(\Gamma_{+}\right)$, mostramos de forma análoga que $f(\delta x)=f(x)$ e portando $f \in \mathcal{P}_{V}(\Gamma)$. Por outro lado, se $f \in \mathcal{P}_{V}(\Gamma)$ temos $f(\delta x)=f(x)$ e então $S_{\Gamma_{+}}^{\Gamma}(f)(x)=0$ para todo $x \in V$ implicando que $f \in \operatorname{ker} S_{\Gamma_{+}}^{\Gamma}$ e portanto

$$
\mathcal{P}_{V}(\Gamma)=\operatorname{ker} S_{\Gamma_{+}}^{\Gamma}
$$

c) Seja $f \in \mathcal{P}_{V}\left(\Gamma_{+}\right)$, pelo item (a) temos $f=R_{\Gamma_{+}}^{\Gamma}(f)+S_{\Gamma_{+}}^{\Gamma}(f)$. Ou seja $f \in \operatorname{Im} R_{\Gamma_{+}}^{\Gamma}+$ $\operatorname{Im} S_{\Gamma_{+}}^{\Gamma}$. É óbvio que $\operatorname{Im} R_{\Gamma_{+}}^{\Gamma}+\operatorname{Im} S_{\Gamma_{+}}^{\Gamma} \subset \mathcal{P}_{V}\left(\Gamma_{+}\right)$, e como $\mathcal{P}_{V}(\Gamma) \cap \mathcal{Q}_{V}(\Gamma)=\{0\}$ segue o resultado.

O resultado abaixo é uma consequência imediata da Proposição 2.3.2 anterior.

Corolário 2.3.3. Existe uma decomposição em soma direta de módulos sobre $\mathcal{P}_{V}(\Gamma)$ para o anel $\mathcal{P}\left(\Gamma_{+}\right)$, dada por

$$
\mathcal{P}_{V}\left(\Gamma_{+}\right)=\mathcal{P}_{V}(\Gamma) \oplus \mathcal{Q}_{V}(\Gamma) .
$$


Lema 2.3.4. Para qualquer $\delta \in \Gamma_{-}$fixado, temos

$$
\overrightarrow{\mathcal{P}}_{V, W}(\Gamma)=\left\{g \in \overrightarrow{\mathcal{P}}_{V, W}\left(\Gamma_{+}\right): g(\delta x)=\delta g(x), \forall x \in V\right\}
$$

$e$

$$
\overrightarrow{\mathcal{Q}}_{V, W}(\Gamma)=\left\{g \in \overrightarrow{\mathcal{P}}_{V, W}\left(\Gamma_{+}\right): g(\delta x)=-\delta g(x), \forall x \in V\right\} .
$$

Demonstração: Temos $\overrightarrow{\mathcal{P}}_{V, W}(\Gamma) \subset \overrightarrow{\mathcal{P}}_{V, W}\left(\Gamma_{+}\right)$pois $\Gamma_{+} \subset \Gamma$. Para todo $\gamma \in \Gamma$ temos ou $\gamma \in \Gamma_{+}$ou $\gamma=\delta \tilde{\gamma}$ para algum $\tilde{\gamma} \in \Gamma_{+}$, ou seja, $\Gamma=\Gamma_{+} \dot{U} \delta \Gamma_{+}$, com $\delta \Gamma_{+}=\Gamma_{-}$.

As inclusões " $\subseteq$ " são consequências diretas das definições de $\overrightarrow{\mathcal{P}}_{V, W}(\Gamma)$ e $\overrightarrow{\mathcal{Q}}_{V, W}(\Gamma)$.

Seja $g \in \overrightarrow{\mathcal{P}}_{V, W}\left(\Gamma_{+}\right)$tal que $g(\delta x)=\delta g(x)$. Se $\gamma \in \Gamma_{+}$então $g(\gamma x)=\gamma g(x)$ pois $g \in \overrightarrow{\mathcal{P}}_{V, W}\left(\Gamma_{+}\right)$. Se $\gamma \in \delta \Gamma_{+}$então

$$
g(\gamma x)=g(\delta \tilde{\gamma} x)=\delta(g \tilde{\gamma} x)=\delta \tilde{\gamma} g(x)=\gamma g(x) \forall x \in V,
$$

mostrando que $g \in \overrightarrow{\mathcal{P}}_{V, W}(\Gamma)$.

Seja $g \in \overrightarrow{\mathcal{P}}_{V, W}\left(\Gamma_{+}\right)$tal que $g(\delta x)=-\delta g(x)$. Se $\gamma \in \Gamma_{+}$então $g(\gamma x)=\gamma g(x)$ pois $g \in \overrightarrow{\mathcal{P}}_{V, W}\left(\Gamma_{+}\right)$. Caso contrário,

$$
g(\gamma x)=g(\delta \tilde{\gamma} x)=-\delta^{-1} g(\tilde{\gamma} x)=-\delta^{-1} \tilde{\gamma} g(x)=\sigma(\gamma) \gamma g(x) \forall x \in V,
$$

e portanto $g \in \overrightarrow{\mathcal{Q}}_{V, W}(\Gamma)$.

Com isso provamos as inclusões " $\supseteq$ " e concluímos as igualdades em (2.26) e (2.27).

Seja $I_{\overrightarrow{\mathcal{P}}_{V}\left(\Gamma_{+}\right.}$a aplicação identidade de $\overrightarrow{\mathcal{P}}_{V}\left(\Gamma_{+}\right)$. Enunciamos abaixo a versão dos resultados anteriores para os operadores $\vec{R}_{\Gamma_{+}}^{\Gamma}$ e $\vec{S}_{\Gamma_{+}}^{\Gamma}$

Proposição 2.3.5. Os operadores de Reynolds $\vec{R}_{\Gamma_{+}}^{\Gamma}$ e $\vec{S}_{\Gamma_{+}}^{\Gamma}$ satisfazem as seguintes propriedades:

a) São homomorfismos entre $\mathcal{P}_{V}(\Gamma)$-módulos e

$$
\vec{R}_{\Gamma_{+}}^{\Gamma}+\vec{S}_{\Gamma_{+}}^{\Gamma}=I_{\overrightarrow{\mathcal{P}}_{V}\left(\Gamma_{+}\right)}
$$

b) São projeções idempotentes com

$$
\begin{aligned}
& \operatorname{ker} \vec{R}_{\Gamma_{+}}^{\Gamma}=\overrightarrow{\mathcal{Q}}_{V, W}(\Gamma) \quad e \quad \operatorname{ker} \vec{S}_{\Gamma_{+}}^{\Gamma}=\overrightarrow{\mathcal{P}}_{V, W}(\Gamma), \\
& \operatorname{Im} \vec{R}_{\Gamma_{+}}^{\Gamma}=\overrightarrow{\mathcal{P}}_{V, W}(\Gamma) \quad e \quad \operatorname{Im} \vec{S}_{\Gamma_{+}}^{\Gamma}=\overrightarrow{\mathcal{Q}}_{V, W}(\Gamma) .
\end{aligned}
$$

c) O anel $\overrightarrow{\mathcal{P}}_{V}\left(\Gamma_{+}\right)$se escreve como uma soma direta de $\mathcal{P}_{V}(\Gamma)$-módulos:

$$
\overrightarrow{\mathcal{P}}_{V}\left(\Gamma_{+}\right)=\operatorname{ker} \vec{R}_{\Gamma_{+}}^{\Gamma} \oplus \operatorname{Im} \vec{R}_{\Gamma_{+}}^{\Gamma}=\operatorname{ker} \vec{S}_{\Gamma_{+}}^{\Gamma} \oplus \operatorname{Im} \vec{S}_{\Gamma_{+}}^{\Gamma}
$$


Demonstração: A prova para essa proposição é semelhente à demonstração da proposição anterior.

a) Para $f \in \mathcal{P}_{V}\left(\Gamma_{+}\right)$e $p \in \overrightarrow{\mathcal{P}}_{V, W}(\Gamma)$ temos

$$
\vec{R}_{\Gamma_{+}}^{\Gamma}(p f)(x)=p(x) \vec{R}_{\Gamma_{+}}^{\Gamma}(f)(x),
$$

$\mathrm{e}$

$$
\vec{R}_{\Gamma_{+}}^{\Gamma}(f+g)(x)=\vec{R}_{\Gamma_{+}}^{\Gamma}(f)(x)+\vec{R}_{\Gamma_{+}}^{\Gamma}(g)(x), \forall x \in V .
$$

Segue que o operador $\vec{R}_{\Gamma_{+}}^{\Gamma}$ é um homomorfismo e de forma análoga mostramos que o $\sigma$-operador $\vec{S}_{\Gamma_{+}}^{\Gamma}$ também é.

A igualdade (2.28) segue direto das definições de $\vec{R}_{\Gamma_{+}}^{\Gamma}$ e $\vec{S}_{\Gamma_{+}}^{\Gamma}$.

b) Agora para qualquer $g \in \overrightarrow{\mathcal{P}}_{V, W}\left(\Gamma_{+}\right)$temos

$$
\vec{R}_{\Gamma_{+}}^{\Gamma}(g)(\delta x)=\frac{1}{2}\left(g(\delta x)+\delta^{-1} g\left(\delta^{2} x\right)\right)=\frac{1}{2}(g(\delta x)+\delta g(x))=\delta \vec{R}_{\Gamma_{+}}^{\Gamma}(g)(x),
$$

$\mathrm{e}$

$$
\vec{S}_{\Gamma_{+}}^{\Gamma}(g)(\delta x)=\frac{1}{2}\left(g(\delta x)-\delta^{-1} g\left(\delta^{2} x\right)\right)=\frac{1}{2}(g(\delta x)-\delta g(x))=-\delta \vec{S}_{\Gamma_{+}}^{\Gamma}(g)(x),
$$

E portanto do Lema 2.3.4 segue que

$$
\vec{R}_{\Gamma_{+}}^{\Gamma}(g) \in \overrightarrow{\mathcal{P}}_{V}(\Gamma) \quad \text { e } \quad \vec{S}_{\Gamma_{+}}^{\Gamma}(g) \in \overrightarrow{\mathcal{Q}}_{V}(\Gamma)
$$

Logo,

$$
\operatorname{Im} \vec{R}_{\Gamma_{+}}^{\Gamma} \subset \overrightarrow{\mathcal{P}}_{V}(\Gamma) \quad \text { e } \quad \operatorname{Im} \vec{S}_{\Gamma_{+}}^{\Gamma} \subset \overrightarrow{\mathcal{Q}}_{V}(\Gamma)
$$

Por outro lado, se $g \in \overrightarrow{\mathcal{P}}_{V, W}(\Gamma)$ então $g(\delta x)=\delta g(x)$. Podemos escrever $g$ na forma

$$
g(x)=\frac{1}{2}\left(g(x)+\delta^{-1} g(\delta x)\right)=\vec{R}_{\Gamma_{+}}^{\Gamma}(g)(x) .
$$

Se $g \in \overrightarrow{\mathcal{Q}}_{V, W}(\Gamma)$ temos $g(\delta x)=-\delta g(x)$. E então

$$
g(x)=\frac{1}{2}\left(g(x)-\delta^{-1} g(\delta x)\right)=\vec{S}_{\Gamma_{+}}^{\Gamma}(g)(x) .
$$

Isto é,

$$
\overrightarrow{\mathcal{P}}_{V, W}(\Gamma) \subset \operatorname{Im} \vec{R}_{\Gamma_{+}}^{\Gamma} \quad \text { e } \quad \mathcal{Q}_{V, W}(\Gamma) \subset \operatorname{Im} \vec{S}_{\Gamma_{+}}^{\Gamma} .
$$

De (2.29) e (2.30) segue que

$$
\overrightarrow{\mathcal{P}}_{V, W}(\Gamma)=\operatorname{Im} \vec{R}_{\Gamma_{+}}^{\Gamma} \quad \text { e } \quad \mathcal{Q}_{V, W}(\Gamma)=\operatorname{Im} \vec{S}_{\Gamma_{+}}^{\Gamma} .
$$


Agora seja $g \in \operatorname{ker}\left(\vec{R}_{\Gamma_{+}}^{\Gamma}\right) \subset \overrightarrow{\mathcal{P}}_{V, W}\left(\Gamma_{+}\right)$e mostremos que $g(\delta x)=-\delta g(x)$. Temos

$$
\vec{R}_{\Gamma_{+}}^{\Gamma}(g)(x)=\frac{1}{2}\left(g(x)+\delta^{-1} g(\delta x)\right)=0,
$$

e portanto $g(\delta x)=-\delta g(x)$ para todo $x \in V$, mostrando que $g \in \overrightarrow{\mathcal{Q}}_{V, W}(\Gamma)$. Por outro lado, se $g \in \overrightarrow{\mathcal{Q}}_{V, W}(\Gamma)$ então $g(\delta x)=-\delta f(x)$ e então $\vec{R}_{\Gamma_{+}}^{\Gamma}(g)(x)=0$ para todo $x \in V$, implicando que $\overrightarrow{\mathcal{Q}}_{V, W}(\Gamma) \subset \operatorname{ker} \vec{R}_{\Gamma_{+}}^{\Gamma}$. Logo

$$
\overrightarrow{\mathcal{Q}}_{V, W}(\Gamma)=\operatorname{ker} \vec{R}_{\Gamma_{+}}^{\Gamma}
$$

Se $g \in \operatorname{ker}\left(\vec{S}_{\Gamma_{+}}^{\Gamma}\right) \subset \overrightarrow{\mathcal{P}}_{V, W}\left(\Gamma_{+}\right)$então $g(\delta x)=\delta g(x)$ e, portando, $g \in \overrightarrow{\mathcal{P}}_{V, W}(\Gamma)$. Por outro lado, se $g \in \overrightarrow{\mathcal{P}}_{V, W}(\Gamma)$ então $g(\delta x)=\delta g(x)$ e então $\vec{S}_{\Gamma_{+}}^{\Gamma}(g)(x)=0$ para todo $x \in V$ implicando que $g \in \operatorname{ker} \vec{S}_{\Gamma_{+}}^{\Gamma}$ e portanto

$$
\overrightarrow{\mathcal{P}}_{V, W}(\Gamma)=\operatorname{ker} \vec{S}_{\Gamma_{+}}^{\Gamma}
$$

c) Se $g \in \overrightarrow{\mathcal{P}}_{V, W}\left(\Gamma_{+}\right)$então pelo item (a) $g=\vec{R}_{\Gamma_{+}}^{\Gamma}(f)+\vec{S}_{\Gamma_{+}}^{\Gamma}(f)$. Ou seja $g \in \operatorname{Im} \vec{R}_{\Gamma_{+}}^{\Gamma}+$ $\operatorname{Im} \vec{S}_{\Gamma_{+}}^{\Gamma}$. Mas $\operatorname{Im} \vec{R}_{\Gamma_{+}}^{\Gamma}+\operatorname{Im} \vec{S}_{\Gamma_{+}}^{\Gamma} \subset \overrightarrow{\mathcal{P}}_{V}\left(\Gamma_{+}\right)$, e como $\overrightarrow{\mathcal{P}}_{V, W}(\Gamma) \cap \overrightarrow{\mathcal{Q}}_{V, W}(\Gamma)=\{0\}$ segue o resultado.

Segue diretamente da proposição acima o seguinte:

Corolário 2.3.6. Existe uma decomposição em soma direta de módulos sobre $\mathcal{P}_{V}(\Gamma)$ para o módulo $\overrightarrow{\mathcal{P}}_{V, W}\left(\Gamma_{+}\right)$, dada por

$$
\overrightarrow{\mathcal{P}}_{V, W}\left(\Gamma_{+}\right)=\overrightarrow{\mathcal{P}}_{V, W}(\Gamma) \oplus \overrightarrow{\mathcal{Q}}_{V, W}(\Gamma)
$$

\subsubsection{Obtenção de formas gerais anti-invariantes}

No Colorário 2.3.3 exibimos uma decomposição para o anel $\mathcal{P}_{V}\left(\Gamma_{+}\right)$como módulo sobre o anel $\mathcal{P}_{V}(\Gamma)$. Tal decomposição é uma soma direta entre dois módulos sobre o anel $\mathcal{P}_{V}(\Gamma)$, onde um é o próprio $\mathcal{P}_{V}(\Gamma)$ e o outro é o módulo dos invariantes $\mathcal{Q}_{V}(\Gamma)$. Nesta subseção apresentamos um método para obter um conjunto de geradores para $\mathcal{Q}_{V}(\Gamma)$ e $\mathcal{P}_{V}(\Gamma)$ como módulos sobre $\mathcal{P}_{V}(\Gamma)$.

Definição 2.3.7. Um multi-índice $\alpha$ é uma s-upla $\alpha=\left(\alpha_{1}, \ldots, \alpha_{s}\right)$ de inteiros não negativos. O grau do multi-índice $\alpha$ é dado por $|\alpha|=\sum_{i=1}^{s} \alpha_{i}$.

Para um vetor $u=\left(u_{1}, \ldots, u_{s}\right)$ usamos a notação $u^{\alpha}$ para representar o produto $u_{1}^{\alpha_{1}} \ldots u_{s}^{\alpha_{s}}$. Também usamos a notação $a_{\alpha}$ para representar $a_{\alpha_{1} \ldots \alpha_{s}}$.

Se $f: V \rightarrow \mathbb{R}$ é um polinômio $\Gamma$-invariante então

$$
f(x)=\sum_{\alpha} a_{\alpha} u^{\alpha}(x), a_{\alpha} \in \mathbb{R} .
$$


Teorema 2.3.8. Seja $\Gamma$ um grupo de Lie compacto agindo em $V$ e seja $\left\{u_{1}, \ldots, u_{s}\right\}$ uma base de Hilbert para o anel $\mathcal{P}_{V}\left(\Gamma_{+}\right)$. Para $i=1, \ldots, s$ e $\delta \in \Gamma_{-}$fixado considere

$$
\tilde{u}_{i}=S_{\Gamma_{+}}^{\Gamma}\left(u_{i}\right)=\frac{1}{2}\left(u_{i}-u_{i} \delta\right) .
$$

Então o conjunto $\left\{\tilde{u}_{1}, \ldots, \tilde{u}_{s}\right\}$ gera o módulo $\mathcal{Q}_{V}(\Gamma)$ sobre o anel $\mathcal{P}_{V}(\Gamma)$.

Observe que o elemento $\tilde{u}_{j}$ é a projecão de $u_{j}$ pelo operador $S_{\Gamma_{+}}^{\Gamma}$ sobre o módulo $\mathcal{Q}_{V}(\Gamma)$.

Demonstração: Seja $\tilde{f} \in Q_{V}(\Gamma)$. Queremos mostrar que existem $p_{j} \in \mathcal{P}_{V}(\Gamma)$ tal que

$$
\tilde{f}(x)=\sum_{j=1}^{s} p_{j}(x) \tilde{u}_{j}(x), \forall x \in V .
$$

Como $Q_{V}(\Gamma)=\operatorname{Im} S_{\Gamma_{+}}^{\Gamma}$, existe $f \in \mathcal{P}_{V}\left(\Gamma_{+}\right)$tal que $\tilde{f}=S_{\Gamma_{+}}^{\Gamma}(f)$, isto é,

$$
\tilde{f}(x)=\frac{1}{2}(f(x)-f(\delta x)) .
$$

Mas $f=\sum b_{\alpha} u^{\alpha}$ com $b_{\alpha} \in \mathbb{R}$ e $\alpha$ um multi-índice. Dessa forma podemos escrever $\tilde{f}$ na forma

$$
\tilde{f}(x)=\sum_{\alpha} a_{\alpha}\left(u^{\alpha}(x)-u^{\alpha}(\delta x)\right)
$$

onde $a_{\alpha} \in \mathbb{R}$.

Façamos indução sobre $s$.

Para $s=1$ temos $\tilde{f}(x)=\sum_{i} a_{i}\left(u^{i}(x)-u^{i}(\delta x)\right)$. Como $u(x)$ e $u(\delta x) \in \mathbb{R}$ vale a igualdade

$$
u^{i}(x)-u^{i}(\delta x)=(u(x)-u(\delta x))\left(\sum_{j=0}^{i-1} u^{j}(x) u^{i-1-j}(\delta x)\right),
$$

observando que

$$
p_{i}(x)=\sum_{j=0}^{i-1} u^{j}(x) u^{i-1-j}(\delta x) \in \mathcal{P}_{V}(\Gamma) .
$$

De fato, como $\Gamma_{+}$é um subgrupo normal de $\Gamma$ segue para todo $\gamma \in \Gamma_{+}$temos $\delta \gamma=\gamma_{1} \delta$ para algum $\gamma_{1} \in \Gamma_{+}$, e então

$$
p_{i}(\gamma x)=\sum_{j=0}^{i-1} u^{j}(\gamma x) u^{i-1-j}\left(\gamma_{1} \delta x\right)=\sum_{j=0}^{i-1} u^{j}(x) u^{i-1-j}(\delta x)=p_{i}(x) .
$$

Agora, se $\gamma \in \delta \Gamma_{+}$então $\gamma=\delta \gamma_{1}=\gamma_{2} \delta \operatorname{com} \gamma_{1}, \gamma_{2}, \delta^{2} \gamma_{1} \in \Gamma_{+}$. Assim,

$$
p_{i}(\gamma x)=\sum_{j=0}^{i-1} u^{j}\left(\gamma_{2} \delta x\right) u^{i-1-j}\left(\delta^{2} \gamma_{1} x\right)=\sum_{j=0}^{i-1} u^{j}(\delta x) u^{i-1-j}(x)=p_{i}(x) .
$$


Portanto obtemos

$$
\tilde{f}(x)=p(x) \tilde{u}(x)
$$

$\operatorname{com} p=\sum_{i} \frac{1}{2} a_{i} p_{i} \in P_{V}(\Gamma)$.

Agora, suponha que para o conjunto $\left\{u_{1}, \ldots, u_{s-1}\right\}$ vale a igualdade

$$
\sum_{\alpha} a_{\alpha}\left(u^{\alpha}(x)-u^{\alpha}(\delta x)\right)=\sum_{j=1}^{s-1} p_{j}(x) \tilde{u}_{j}(x),
$$

$\operatorname{com} \alpha=\left(\alpha_{1}, \ldots, \alpha_{s-1}\right)$.

Agora, considere a base de Hilbert $\left\{u_{1}, \ldots, u_{s}\right\}=\left\{u_{1}, \ldots, u_{s-1}\right\} \cup\left\{u_{s}\right\}$, para o anel $P_{V}\left(\Gamma_{+}\right)$. Seguindo o mesmo raciocínio de (2.34) segue que

$$
\tilde{f}(x)=\sum_{\alpha, \alpha_{s}} a_{\alpha \alpha_{s}}\left(u^{\alpha}(x) u_{s}^{\alpha_{s}}(x)-u^{\alpha}(\delta x) u_{s}^{\alpha_{s}}(\delta x)\right) .
$$

Observe que $u^{\alpha}, u^{\alpha_{s}} \in \mathcal{P}_{V}\left(\Gamma_{+}\right)=\mathcal{P}_{V}(\Gamma) \oplus \mathcal{Q}_{V}(\Gamma)$. Então

$$
u^{\alpha}(x)=v_{\alpha}(x)+w_{\alpha}(x) \quad \text { e } \quad u^{\alpha_{s}}(x)=v_{\alpha_{s}}(x)+w_{\alpha_{s}}(x),
$$

$\operatorname{para} v_{\alpha}, v_{\alpha_{s}} \in \mathcal{P}_{V}(\Gamma)$ e $w_{\alpha}, w_{\alpha_{s}} \in \mathcal{Q}_{V}(\Gamma)$.

Então

$$
u^{\alpha}(\delta x)=v_{\alpha}(x)-w_{\alpha}(x) \quad \text { e } \quad u^{\alpha_{s}}(\delta x)=v_{\alpha_{s}}(x)-w_{\alpha_{s}}(x),
$$

e portanto

$$
u^{\alpha}(x)-u^{\alpha}(\delta x)=2 w_{\alpha}(x) \quad \text { e } \quad u_{s}^{\alpha_{s}}(x)-u_{s}^{\alpha_{s}}(\delta x)=2 w_{\alpha_{s}}(x) .
$$

Reescrevendo (2.37) obtemos

$$
\begin{aligned}
\tilde{f}(x) & =\sum_{\alpha, \alpha_{s}} a_{\alpha \alpha_{s}}\left[u^{\alpha}(x) u_{s}^{\alpha_{s}}(x)+u^{\alpha}(x) u_{s}^{\alpha_{s}}(\delta x)-u^{\alpha}(x) u_{s}^{\alpha_{s}}(\delta x)-u^{\alpha}(\delta x) u_{s}^{\alpha_{s}}(\delta x)\right] \\
& =\sum_{\alpha, \alpha_{s}} a_{\alpha \alpha_{s}}\left[u^{\alpha}(x)\left(u_{s}^{\alpha_{s}}(x)-u_{s}^{\alpha_{s}}(\delta x)\right)+u_{s}^{\alpha_{s}}(\delta x)\left(u^{\alpha}(x)-u^{\alpha}(\delta x)\right)\right] \\
& =\sum_{\alpha, \alpha_{s}} a_{\alpha \alpha_{s}}\left[\left(v_{\alpha}(x)+w_{\alpha}(x)\right) 2 w_{\alpha_{s}}(x)+\left(v_{\alpha_{s}}(x)-w_{\alpha_{s}}(x)\right) 2 w_{\alpha}(x)\right] \\
& =\sum_{\alpha, \alpha_{s}} a_{\alpha \alpha_{s}}\left[2 v_{\alpha}(x) w_{\alpha_{s}}(x)+2 v_{\alpha_{s}}(x) w_{\alpha}(x)\right] \\
& =\sum_{\alpha, \alpha_{s}} a_{\alpha \alpha_{s}}[v_{\alpha}(x)\left(u_{s}^{\alpha_{s}}(x)-u_{s}^{\alpha_{s}}\right)(x)+\underbrace{\left.v_{\alpha_{s}}(x)\left(u^{\alpha}(x)-u^{\alpha}(\delta x)\right)\right]}_{(I)} \\
= & \sum_{\alpha, \alpha_{s}} a_{\alpha \alpha_{s}} v_{\alpha}(x)\left(u_{s}^{\alpha_{s}}(x)-u_{s}^{\alpha_{s}}(\delta x)\right)+\underbrace{\sum_{\alpha \alpha_{s}} v_{\alpha_{s}}(x)\left(u^{\alpha}(x)-u^{\alpha}(\delta x)\right)}_{\alpha_{s}} .
\end{aligned}
$$

Mas

$$
(I)=\sum_{\alpha_{s}}\left(\sum_{\alpha} a_{\alpha \alpha_{s}} v_{\alpha_{s}}(x)\left(u^{\alpha}(x)-u^{\alpha}(\delta x)\right)\right)
$$




$$
=\sum_{\alpha_{s}} v_{\alpha_{s}}(x) \sum_{\alpha} a_{\alpha \alpha_{s}}\left(u^{\alpha}(x)-u^{\alpha}(\delta x)\right)=\sum_{\alpha_{s}} v_{\alpha_{s}}(x) \sum_{j=1}^{s-1} p_{j}(x) \tilde{u}_{j}(x),
$$

sendo a última igualdade proveniente da hipótese de indução. De (2.35) segue que existe $p_{s} \in \mathcal{P}_{V}(\Gamma)$ tal que

$$
a_{\alpha \alpha_{s}}\left(u_{s}^{\alpha_{s}}(x)-u_{s}^{\alpha_{s}}(\delta x)\right)=p_{s}(x) \tilde{u}_{s}(x) .
$$

Então podemos reescrever a igualdade (2.38) da seguinte forma

$$
\begin{aligned}
\tilde{f}(x) & =\sum_{\alpha} v_{\alpha}(x) p_{s}(x) \tilde{u}_{s}(x)+\sum_{\alpha_{s}} v_{\alpha_{s}}(x) \sum_{j=1}^{s-1} p_{j}(x) \tilde{u}_{j}(x) \\
& =\left(\sum_{\alpha} v_{\alpha}(x) p_{s}(x)\right) \tilde{u}_{s}(x)+\sum_{j=1}^{s-1}\left(\sum_{\alpha_{s}} v_{\alpha_{s}}(x) p_{j}(x)\right) \tilde{u}_{j}(x) \\
& =\sum_{j=1}^{s} \bar{p}_{j}(x) \tilde{u}_{j}(x)
\end{aligned}
$$

onde

$$
\bar{p}_{j}(x)=\sum_{\alpha_{s}} v_{\alpha_{s}}(x) p_{j}(x), \forall 1 \leq j \leq s-1
$$

$\mathrm{e}$

$$
\bar{p}_{s}(x)=\sum_{\alpha} v_{\alpha}(x) p_{s}(x),
$$

demonstrando o resultado.

Corolário 2.3.9. Seja $\Gamma$ um grupo de Lie compacto agindo em $V$ e seja $\left\{u_{1}, \ldots, u_{s}\right\}$ uma base de Hilbert para o anel $\mathcal{P}_{V}\left(\Gamma_{+}\right)$. Para $i=1, \ldots, s$ e $\delta \in \Gamma_{-}$fixado considere

$$
\tilde{u}_{i}=S_{\Gamma_{+}}^{\Gamma}\left(u_{i}\right)=\frac{1}{2}\left(u_{i}-u_{i} \delta\right) .
$$

Então o conjunto $\left\{1, \tilde{u}_{1}, \ldots, \tilde{u}_{s}\right\}$ gera $\mathcal{P}_{V}\left(\Gamma_{+}\right)$como módulo sobre o anel $\mathcal{P}_{V}(\Gamma)$.

Demonstração: A demonstração é decorrente da decomposição do anel $\mathcal{P}_{V}\left(\Gamma_{+}\right)$como soma direta dos módulos $\mathcal{P}_{V}(\Gamma)$ e $\mathcal{Q}_{V}(\Gamma)$ sobre o anel $\mathcal{P}_{V}(\Gamma)$ dada no Corolário 2.3.3.

\section{Algoritmo 2.3.10. (Geradores para os anti-invariantes)}

1. Considere um grupo de Lie compacto $\Gamma$;

2. Defina um subgrupo normal $\Gamma_{+} \subset \Gamma$ de indice 2;

3. Fixe uma anti-simetria $\delta \in \Gamma_{-}$;

4. Tome uma base de Hilbert homogênea $\left\{u_{1}, \ldots, u_{s}\right\}$ para $\mathcal{P}_{V}\left(\Gamma_{+}\right)$;

5. Para $i=1$ até s faça

$$
\tilde{u}_{i}(x)=\frac{1}{2}\left(u_{i}(x)-u_{i}(\delta x)\right) .
$$

Resultado: Obtenção do conjunto gerador homogênio $\left\{\tilde{u}_{1}, \ldots, \tilde{u}_{s}\right\}$ para o módulo $\mathcal{Q}_{V}(\Gamma)$ sobre $\mathcal{P}_{V}(\Gamma)$. 


\subsubsection{Obtenção de formas gerais reversíveis-equivariantes}

No Corolário 2.3.6 obtemos uma decomposição para o módulo $\overrightarrow{\mathcal{P}}_{V, W}\left(\Gamma_{+}\right)$sobre o anel $\mathcal{P}_{V, W}(\Gamma)$, em uma soma de dois módulos, onde um é $\overrightarrow{\mathcal{P}}_{V, W}(\Gamma)$ e o outro é o $\overrightarrow{\mathcal{Q}}_{V, W}(\Gamma)$. Agora, mostramos como obter um conjunto de geradores para os módulos $\overrightarrow{\mathcal{P}}_{V, W}(\Gamma)$ e $\overrightarrow{\mathcal{Q}}_{V, W}(\Gamma)$ sobre o anel $\mathcal{P}_{V}(\Gamma)$.

Em nosso estudo temos que $\overrightarrow{\mathcal{P}}_{V, W}\left(\Gamma_{+}\right)$é um módulo sobre $\mathcal{P}_{V}\left(\Gamma_{+}\right)$e que $\mathcal{P}_{V}\left(\Gamma_{+}\right)$é um módulo sobre $\mathcal{P}_{V}(\Gamma)$. Como vimos no Corolário 2.3.9 se $\left\{u_{1}, \ldots, u_{s}\right\}$ é uma base de Hilbert para o anel $\mathcal{P}_{V}(\Gamma)$ então $\left\{1, \tilde{u}_{1}, \ldots, \tilde{u}_{s}\right\}$ é um conjunto gerador para o módulo $\mathcal{P}_{V}\left(\Gamma_{+}\right)$sobre o anel $\mathcal{P}_{V}(\Gamma)$. Então é natural o seguinte lema:

Lema 2.3.11. Seja $\Gamma$ um grupo de Lie compacto agindo em $V$ e seja $\left\{u_{1}, \ldots, u_{s}\right\}$ uma base de Hilbert para o anel $\mathcal{P}_{V}\left(\Gamma_{+}\right)$. Considere o conjunto gerador $\left\{\tilde{u}_{0}=1, \tilde{u}_{1}, \ldots, \tilde{u}_{s}\right\}$ para o módulo $\mathcal{P}_{V}\left(\Gamma_{+}\right)$sobre o anel $\mathcal{P}_{V}(\Gamma)$, e seja $\left\{H_{0}, \ldots, H_{r}\right\}$ um conjunto gerador para o módulo $\overrightarrow{\mathcal{P}}_{V, W}\left(\Gamma_{+}\right)$sobre $\mathcal{P}_{V}\left(\Gamma_{+}\right)$. Para $i=0, \ldots, s$ e $j=0, \ldots, r$ considere

$$
H_{i j}=\tilde{u}_{i} H_{j}
$$

Então o conjunto $\left\{H_{i j}, \ldots, H_{r s}\right\}$ gera $\overrightarrow{\mathcal{P}}_{V, W}\left(\Gamma_{+}\right)$como módulo sobre o anel $\mathcal{P}_{V}(\Gamma)$.

Teorema 2.3.12. Seja $\Gamma$ um grupo de Lie compacto agindo em $V$ e seja $\left\{H_{00}, \ldots, H_{s r}\right\}$ um conjunto gerador para o módulo $\overrightarrow{\mathcal{P}}_{V, W}\left(\Gamma_{+}\right)$sobre $\mathcal{P}_{V}(\Gamma)$. Para $i=0, \ldots$, s e $j=$ $0, \ldots, r$ considere

$$
\tilde{H}_{i j}=\vec{S}_{\Gamma_{+}}^{\Gamma}\left(H_{i j}\right) \text {. }
$$

Então o conjunto $\left\{\tilde{H}_{00}, \ldots, \tilde{H}_{r s}\right\}$ gera $\overrightarrow{\mathcal{Q}}_{V, W}(\Gamma)$ como módulo sobre o anel $\mathcal{P}_{V}(\Gamma)$.

Demonstração: Se $\tilde{f} \in \overrightarrow{\mathcal{Q}}_{V, W}(\Gamma)=\operatorname{Im} \vec{S}_{\Gamma_{+}}^{\Gamma}$, existe $f \in \overrightarrow{\mathcal{P}}_{V, W}\left(\Gamma_{+}\right)$tal que $\tilde{f}=\vec{S}_{\Gamma_{+}}^{\Gamma}(f)$. Como $f \in \overrightarrow{\mathcal{P}}_{V, W}\left(\Gamma_{+}\right)$, existem $p_{i j} \in \mathcal{P}_{V}(\Gamma)$ tal que

$$
f=\sum_{i, j=0}^{s, r} p_{i j} H_{i j}
$$

Segue que

$$
\tilde{f}=\vec{S}_{\Gamma_{+}}^{\Gamma}\left(\sum_{i, j=0}^{s, r} p_{i j} H_{i j}\right)=\sum_{i, j=0}^{s, r} p_{i j} \vec{S}_{\Gamma_{+}}^{\Gamma}\left(H_{i j}\right)=\sum_{i, j=0}^{s, r} p_{i j} \tilde{H}_{i j} .
$$

Observe então que para a construção de um conjunto de geradores para o módulo dos reversíveis-equivariantes, basta conhecer uma base de Hilbert para o anel $\mathcal{P}_{V}(\Gamma)$ e um conjunto de geradores para $\overrightarrow{\mathcal{P}}_{V, W}\left(\Gamma_{+}\right)$como módulo sobre $\mathcal{P}_{V}(\Gamma)$. Dessa teoria segue o seguinte algorítmo:

\section{Algoritmo 2.3.13. (Geradores para os reversíveis-equivariantes)}

1. Considere um grupo de Lie compacto $\Gamma$; 
2. Defina um subgrupo normal $\Gamma_{+} \subset \Gamma$ de indice 2;

3. Fixe uma anti-simetria $\delta \in \Gamma_{-}$;

4. Tome uma base de Hilbert homogênea $\left\{u_{1}, \ldots, u_{s}\right\}$ para $\mathcal{P}_{V}\left(\Gamma_{+}\right)$;

5. Determine um conjunto $\left\{H_{0}, \ldots, H_{r}\right\}$ de geradores homogênios para $\overrightarrow{\mathcal{P}}_{V, W}\left(\Gamma_{+}\right)$ como módulo sobre $\mathcal{P}_{V}\left(\Gamma_{+}\right)$e defina $\tilde{u}_{0}=1$

6. Para $i=1$ até s faça

$$
\tilde{u}_{i}(x)=\frac{1}{2}\left(u_{i}(x)-u_{i}(\delta x)\right)
$$

Para $j=0$ até $r$ faça

$$
\begin{aligned}
H_{0 j}(x) & =H_{j}(x) \\
H_{i j}(x) & =\tilde{u}_{j}(x) H_{j}(x) \\
\tilde{H}_{i j}(x) & =\frac{1}{2}\left(H_{i j}(x)-\delta^{-1} H_{i j}(\delta x)\right) .
\end{aligned}
$$

Resultado: Obtenção do conjunto gerador homogênio $\left\{\tilde{H}_{00}, \ldots, \tilde{H}_{\text {sr }}\right\}$ para o módulo $\overrightarrow{\mathcal{Q}}_{V, W}(\Gamma)$ sobre $\mathcal{P}_{V}(\Gamma)$

Observação 2.3.14. Se $H_{i j} \in \overrightarrow{\mathcal{P}}_{V, W}(\Gamma)=\operatorname{ker} \vec{S}_{\Gamma_{+}}^{\Gamma}$, ent $\tilde{a} O \tilde{H}_{i j} \equiv 0$.

\subsection{O Pêndulo simples}

Para motivar os conceitos vistos neste capítulo analisamos a equação do pêndulo simples, num plano sob à ação da força da gravidade $G$. Supomos o pêndulo como na Figura 2.2, de haste rígida de comprimento $l$ e massa desprezível, com um extremo fixo e uma pequena esfera de massa $m$ no outro extremo.

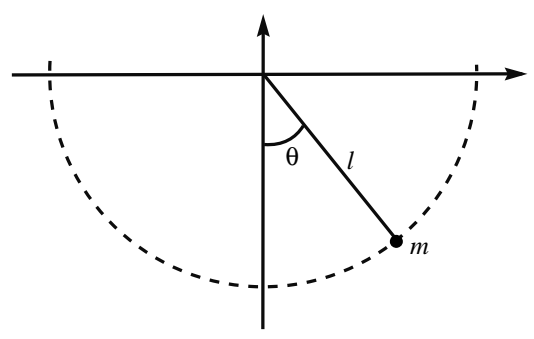

Figura 2.2: O pêndulo.

Considerando o caso de atrito desprezível, a equação de movimento do pêndulo é dada por

$$
\ddot{\theta}=\frac{d^{2} \theta}{d t^{2}}=-\frac{G}{l} \operatorname{sen} \theta
$$


Passamos da EDO de $2^{\text {a }}$ ordem em (2.40), para um sistema de EDO's de $1^{\mathrm{a}}$ ordem:

$$
\begin{aligned}
& \dot{\theta}=\omega \\
& \dot{\omega}=-\frac{G}{l} \operatorname{sen} \theta .
\end{aligned}
$$

O retrato de fase do sistema acima está ilustrado na figura 2.3.

Decorre que se $\theta$ é uma solução de (2.40), então o caminho $(\theta, \omega): I \subseteq \mathbb{R} \rightarrow \mathbb{R}^{2}$, o qual é uma trajetória do sistema mecânico dado pelo pêndulo, define uma curva no plano $\theta \times \omega$, que está inteiramente contida numa curva de nível da energia total.

Finalmente, analisamos que o campo de vetores

$$
g(\theta, \omega)=\left(\omega,-\frac{G}{l} \operatorname{sen} \theta\right)
$$

é $\mathbf{D}_{2}$-reversível-equivariante com anti-simetria $\kappa$.

De fato, como $\mathbf{D}_{2}=\left\langle R_{\pi}, \kappa\right\rangle$, sendo $\kappa$ definido em (1.1), basta observar que

$$
\begin{aligned}
g(\kappa(\theta, \omega)) & =g(\theta,-\omega)=\left(-\omega,-\frac{G}{l} \operatorname{sen} \theta\right)=-\left(\omega, \frac{G}{l} \operatorname{sen} \theta\right) \\
& =-\kappa\left(\omega,-\frac{G}{l} \operatorname{sen} \theta\right)=-\kappa g(\theta, \omega),
\end{aligned}
$$

e

$$
\begin{aligned}
g\left(R_{\pi}(\theta, \omega)\right) & =g(-\theta,-\omega)=-\left(\omega, \frac{G}{l} \operatorname{sen}(-\theta)\right)=\left(-\omega, \frac{G}{l} \operatorname{sen} \theta\right) \\
& =R_{\pi}\left(\omega,-\frac{G}{l} \operatorname{sen} \theta\right)=R_{\pi} g(\theta, \omega) .
\end{aligned}
$$

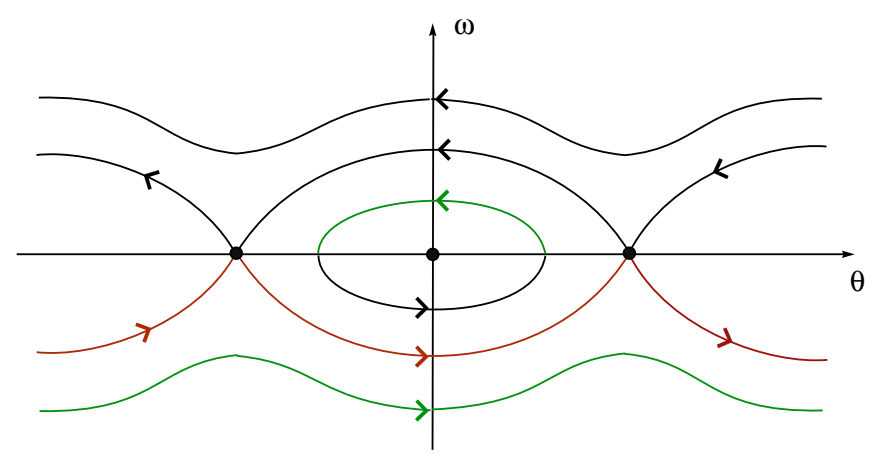

Figura 2.3: Retrato de fase do pêndulo sem atrito. 



\section{Capítulo 3}

\section{Produto Coroa}

Neste capítulo adaptamos a representação de grupos para o estudo sistemático de um sistema de células idênticas acopladas, cada uma representando um sistema $\Gamma$-reversívelequivariante definido num espaço vetorial $V$, com $\Gamma$ grupo de Lie compacto. Supomos $N$ células, cada uma acoplada com as demais (acoplamento um-a-um), de forma que as simetrias e anti-simetrias que regem todo o sistema é dado pelo produto coroa $\Gamma \imath \mathbf{S}_{N}$ de $\Gamma$ por $\mathbf{S}_{N}$ (veja Definição 3.1.2), onde $\mathbf{S}_{N}$ é o grupo das permutações de $N$ elementos.

Se cada célula é representada por uma EDO do tipo $\dot{x}=f(x), x \in V$, então um acoplamento destas células é modelado por um sistema da forma:

$$
\begin{gathered}
\dot{x}_{1}=f\left(x_{1}\right)+h_{1}(x), \\
\vdots \\
\dot{x}_{N}=f\left(x_{N}\right)+h_{N}(x),
\end{gathered}
$$

com $x=\left(x_{1}, \ldots, x_{N}\right) \in V^{N}$ e $h=\left(h_{1}, \ldots, h_{N}\right)$ a aplicação do acoplamento.

O acoplamento de células puramente equivariantes foi tratado primeiramente por Dionne et al [10] e por vários outros autores desde então. Neste caso, $\Gamma$ é um grupo de simetrias apenas, chamadas simetrias internas; as simetrias presentes no acoplamento são chamadas simetrias globais, digamos do grupo $\mathbf{S}_{N}$. Em geral, as simetrias internas e globais podem se combinar de duas formas distintas dependendo de como as simetrias internas afetam o acoplamento, resultando algebricamente no produto coroa $\Gamma$ < $\mathbf{S}_{N}$ ou no produto direto $\Gamma \times \mathbf{S}_{N}$. Aqui consideramos o produto coroa que ocorre quando o acoplamento das células é invariante pela ação das simetrias internas. Em particular, como já foi dito acima, vamos assumir acoplamento um-a-um de células idênticas que são $\Gamma$-reversíveis-equivariantes. Nosso objetivo é descrever a ação apropriada de $\Gamma$ 乙 $\mathbf{S}_{N}$ em $V^{N}$, apresentado na Seção 3.2. Na Seção 3.1 resumimos a teoria de acoplamento de células equivariantes e na Seção 3.2 apresentamos o acoplamento de células reversíveisequivariantes, incluindo um exemplo da ação de $\mathbf{O}(2)$ 々 $\mathbf{S}_{N}$ em $\mathbb{C}^{N}$.

\section{$3.1 \quad \mathrm{O}$ produto coroa $\Gamma \imath \mathrm{S}_{N}$ como grupo de simetrias}

Como sempre consideramos $\Gamma$ um grupo de Lie compacto agindo em um espaço vetorial real $V$ de dimensão finita. 
Induzimos uma ação diagonal de $\Gamma^{N}$ em $V^{N}$ por

$$
\gamma\left(v_{1}, \ldots, v_{N}\right)=\left(\gamma_{1} v_{1}, \ldots, \gamma_{N} v_{N}\right)
$$

para $\gamma=\left(\gamma_{1}, \ldots, \gamma_{N}\right) \in \Gamma^{N}$

Para continuar nosso estudo, passamos à definição de produto semi-direto:

Definição 3.1.1. Sejam $\mathcal{K}$ e $\mathcal{G}$ grupos e a aplicação $\varphi: \mathcal{G} \rightarrow \operatorname{Aut}(\mathcal{K})$ um homomorfismo. Então o conjunto de todos os pares ordenados $(k, \tau), k \in \mathcal{K}$ e $\tau \in G$, forma um grupo, a soma semi-direta de $\mathcal{K}$ por $\mathcal{G}$, denotada por $\mathcal{K} \dot{+} G$, com a operação de multiplicação

$$
(k, \tau)\left(k_{1}, \tau_{1}\right)=\left(k \varphi(\tau)\left(k_{1}\right), \tau \tau_{1}\right) .
$$

Para nossa finalidade definimos:

Definição 3.1.2. O produto coroa de $\Gamma$ por $\boldsymbol{S}_{N}$ é a soma semi-direta de $\Gamma^{N}$ por $\boldsymbol{S}_{N}$, e é denotado por $\Gamma$ 々 $\boldsymbol{S}_{N}$.

Com a ação do grupo $\mathbf{S}_{N}$ das permutações em $V^{N}$ dada por

$$
\tau\left(v_{1}, \ldots, v_{n}\right)=\left(v_{\tau^{-1}(1)}, \ldots, v_{\tau^{-1}(N)}\right),
$$

para $\left(v_{1}, \ldots, v_{N}\right) \in V^{N}$ e $\tau \in \mathbf{S}_{N}$, consideramos a ação de $\Gamma \imath \mathbf{S}_{N}$ em $V^{N}$ dada por

$$
(\gamma, \tau)\left(v_{1}, \ldots, v_{n}\right)=\left(\gamma_{1} v_{\tau^{-1}(1)}, \ldots, \gamma_{N} v_{\tau^{-1}(N)}\right),
$$

para $\gamma=\left(\gamma_{1}, \ldots, \gamma_{N}\right) \in \Gamma^{N},\left(v_{1}, \ldots, v_{N}\right) \in V^{N}$ e $\tau \in \mathbf{S}_{N}$.

Observe que a operação acima é uma ação. De fato, como

$$
(\gamma, \tau)[(\delta, \varrho) v]=(\gamma, \tau)\left(\sigma\left(\delta_{1}\right) \delta_{1} v_{\varrho^{-1}(1)}, \ldots, \sigma\left(\delta_{N}\right) \delta_{N} v_{\varrho^{-1}(N)}\right),
$$

tomamos $y_{i}=\sigma\left(\delta_{i}\right) \delta_{i} v_{\varrho^{-1}(i)}$ e obtemos

$$
\begin{aligned}
(\gamma, \tau)[(\delta, \varrho) v] & =(\gamma, \tau)\left(y_{1}, \ldots, y_{N}\right) \\
& =\left(\gamma_{1} y_{\varrho^{-1}(1)}, \ldots, \gamma_{N} y_{\varrho^{-1}(N)}\right) \\
& =\left(\gamma_{1} \delta_{\tau^{-1}(1)} v_{\varrho^{-1}\left(\varrho^{-1}(1)\right)}, \ldots, \gamma_{N} \delta_{\tau^{-1}(N)} v_{\varrho^{-1}\left(\varrho^{-1}(N)\right)}\right. \\
& \left.=\gamma_{1} \delta_{\tau^{-1}(1)} v_{(\tau \varrho)^{-1}(1)}, \ldots, \gamma_{N} \delta_{\tau^{-1}(N)} v_{(\tau \varrho)^{-1}(N)}\right) \\
& =[(\gamma, \tau)(\delta, \varrho)] v .
\end{aligned}
$$

Ao longo desta seção a operação * denota o produto das componentes de mesmo índices de dois vetores, ou seja, denotamos por $z * w$ todo elemento da forma $\left(z_{1} w_{1}, \ldots, z_{N} w_{N}\right)$ para $z=\left(z_{1}, \ldots, z_{N}\right), w=\left(w_{1}, \ldots, w_{N}\right) \in \mathbb{C}^{N}$. Lembramos que se $\alpha$ é um multi-índice então $z^{\alpha}$ denota o produto $z_{1}^{\alpha_{1}} \ldots z_{N}^{\alpha_{N}}$ e $a_{\alpha}$ denota $a_{\alpha_{1} \ldots \alpha_{N}}$.

\subsubsection{Geradores dos equivariantes para o grupo $\mathrm{O}(2) \succ \mathbf{S}_{N}$}

Para $\Gamma=\mathbf{O}(2)$, considere a ação padrão do grupo $\mathbf{O}(2)$ em $\mathbb{C}$ definida no Exemplo 1.1.5(4) e a ação diagonal de $\mathbf{O}(2)^{N}$ em $\mathbb{C}^{N}$ definida em (3.1).

Começamos enunciando dois lemas, os quais nos fornece uma base de Hilbert para o anel $\mathcal{P}_{V^{N}}\left(\mathbf{S}_{N}\right)$ e um conjunto de geradores para $\overrightarrow{\mathcal{P}}_{V^{N}}\left(\mathbf{S}_{N}\right)$ como módulo sobre $\mathcal{P}_{V^{N}}\left(\mathbf{S}_{N}\right)$. 
Lema 3.1.3. Considere a ação de $\boldsymbol{S}_{N}$ em $V^{N}$. Então o conjunto

$$
\left\{x_{1}+\ldots+x_{N}, x_{1}^{2}+\ldots+x_{N}^{2}, \ldots, x_{1}^{N}+\ldots+x_{N}^{N}\right\},
$$

é uma base de Hilbert para o anel $\mathcal{P}_{V^{N}}\left(\boldsymbol{S}_{N}\right)$.

Demonstração: Veja [19], Capítulo 3, Proposição 3.2.4.

Lema 3.1.4. O módulo $\overrightarrow{\mathcal{P}}_{V^{N}}\left(\boldsymbol{S}_{N}\right)$ é gerado sobre o anel $\mathcal{P}_{V^{N}}\left(\boldsymbol{S}_{N}\right)$ pelas aplicações

$$
\left(\begin{array}{c}
x_{1}^{k} \\
x_{2}^{k} \\
\vdots \\
x_{N}^{k}
\end{array}\right), \text { para } k=0, \ldots, N-1 .
$$

Demonstração: Veja Dias [9], Appendix B.

Lema 3.1.5. Considere a ação de $\boldsymbol{O}(2)^{N}$ em $\mathbb{C}^{N}$. Então o conjunto

$$
\left\{z_{1} \bar{z}_{1}, \ldots, z_{N} \bar{z}_{N}\right\}
$$

é uma base de Hilbert para o anel $\mathcal{P}_{\mathbb{C}^{N}}\left(\boldsymbol{O}(2)^{N}\right)$.

Demonstração: Seja $p: \mathbb{C}^{N} \rightarrow \mathbb{R}$ polinômio $\mathbf{O}(2)^{N}$-invariante. Escrevemos $p$ nas coordenadas $z$ e $\bar{z}$ na forma

$$
p(z)=\sum a_{\alpha \beta} z^{\alpha} \bar{z}^{\beta}
$$

com $a_{\alpha \beta} \in \mathbb{C}$, e $\alpha, \beta$ multi-índices. Como $p$ é polinômio real, temos $p=\bar{p}$ e, portanto,

$$
\bar{a}_{\alpha \beta}=a_{\alpha \beta} .
$$

Para todo $\theta=\left(\theta_{1}, \ldots, \theta_{N}\right) \in \mathbf{O}(2)^{N}$ temos $p(\theta z)=p(z)$, isto é,

$$
p\left(e^{i \theta_{1}} z_{1}, \ldots, e^{i \theta_{N}} z_{N}\right)=p\left(z_{1}, \ldots, z_{N}\right) .
$$

Então,

$$
\sum a_{\alpha \beta}\left(e^{i \theta} z\right)\left(\overline{e^{i \theta} z}\right)^{\beta}=\sum a_{\alpha \beta} e^{i\left[\theta_{1}\left(\alpha_{1}-\beta_{1}\right)+\ldots+\theta_{N}\left(\alpha_{N}-\beta_{N}\right)\right]} z^{\alpha} \bar{z}^{\beta}=p(z),
$$

implicando em,

$$
a_{\alpha \beta}=a_{\alpha \beta} e^{i\left[\theta_{1}\left(\alpha_{1}-\beta_{1}\right)+\ldots+\theta_{N}\left(\alpha_{N}-\beta_{N}\right)\right]}, \forall \theta=\left(\theta_{1}, \ldots, \theta_{N}\right) \in \mathbf{O}(2)^{N} .
$$

Segue que $a_{\alpha \beta}=0$ ou $\alpha_{1}=\beta_{1}, \ldots, \alpha_{N}=\beta_{N}(\alpha=\beta)$.

Reescrevendo (3.4), com $a_{\alpha \alpha}=a_{\alpha}$, obtemos

$$
p(z)=\sum_{\alpha} a_{\alpha}(z \bar{z})^{\alpha}=\sum_{\alpha} a_{\alpha}(z \bar{z})^{\alpha_{1}} \ldots(z \bar{z})^{\alpha_{N}} .
$$

Segue de (3.5) que $a_{\alpha} \in \mathbb{R}$ e, então, provamos o lema. 
Lema 3.1.6. Se o polinômio $p: V^{N} \rightarrow \mathbb{R}$ é $\Gamma$ 々 $\boldsymbol{S}_{N}$-invariante, então p também é $\Gamma^{N}$-invariante e $\boldsymbol{S}_{N}$-invariante.

Demonstração: Se $p \in \mathcal{P}_{V^{N}}\left(\Gamma \imath \mathbf{S}_{N}\right)$, então $p((\gamma, \tau) \cdot v)=p(v)$ para todo $v \in V^{N} \mathrm{e}$

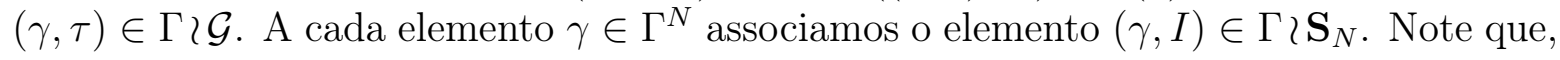

$$
p(\gamma v)=p((\gamma, I) \cdot v)=p(v)
$$

$\operatorname{Logo} p \in \mathcal{P}_{V^{N}}\left(\Gamma^{N}\right)$.

De forma análoga a cada elemento de $\tau \in \mathbf{S}_{N}$ associamos o elemento $(I, \tau) \in \Gamma$ $\mathcal{G}$, e o resultado segue.

Teorema 3.1.7. Se $\left\{\mu_{1}, \ldots, \mu_{s}\right\}$ é uma base de Hilbert para o anel $\mathcal{P}_{\mathbb{C}^{N}}\left(\boldsymbol{S}_{N}\right)$, então

$$
\left\{\mu_{1}\left(z_{1} \overline{z_{1}}, \ldots, z_{N} \overline{z_{N}}\right), \ldots, \mu_{s}\left(z_{1} \overline{z_{1}}, \ldots, z_{N} \overline{z_{N}}\right)\right\}
$$

é uma base de Hilbert para $\mathcal{P}_{\mathbb{C}^{N}}\left(\boldsymbol{O}(2)<\boldsymbol{S}_{N}\right)$.

Demonstração: Pelo Teorema de Hilbert-Weyl (Teorema 1.3.5), existe uma base finita $\left\{\mu_{1}, \ldots, \mu_{s}\right\}$ para o anel $\mathcal{P}_{\mathbb{C}^{N}}\left(\mathbf{S}_{N}\right)$. Seja $p: \mathbb{C}^{N} \rightarrow \mathbb{R} \mathbf{O}(2)<\mathbf{S}_{N^{-}}$invariante. Pelo Lema 3.1.6, $p$ é $\mathbf{O}(2)^{N}$-invariante, e pelo Lema 3.1.5, existe $h: \mathbb{R}^{N} \rightarrow \mathbb{R}$ tal que $p(z)=h\left(z_{1} \overline{z_{1}}, \ldots, z_{N} \overline{z_{N}}\right)$.

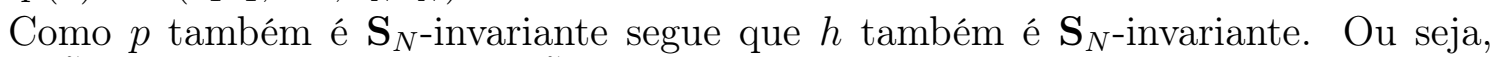
existe $\tilde{h}: \mathbb{R}^{s} \rightarrow \mathbb{R}$ tal que $h(x)=\tilde{h}\left(\mu_{1}(x), \ldots, \mu_{s}(x)\right)$, para $x \in \mathbb{R}^{N}$. Então

$$
p(z)=h(z * \bar{z})=\tilde{h}\left(\mu(z * \bar{z}), \ldots, \mu_{s}(z * \bar{z})\right),
$$

para todo $z \in \mathbb{C}^{N}$ provando o resultado.

Lema 3.1.8. Considere as aplicações

$$
g_{1}(z)=\left(z_{1}, 0, \ldots, 0\right), \ldots, g_{N}(z)=\left(0, \ldots, 0, z_{N}\right)
$$

Então o conjunto $\left\{g_{1}, \ldots, g_{N}\right\}$ gera o módulo $\overrightarrow{\mathcal{P}}_{\mathbb{C}^{N}}\left(\boldsymbol{O}(2)^{N}\right)$ sobre $\mathcal{P}_{\mathbb{C}^{N}}\left(\boldsymbol{O}(2)^{N}\right)$.

Demonstração: Seja $q \in \overrightarrow{\mathcal{P}}_{\mathbb{C}^{N}}\left(\mathbf{O}(2)^{N}\right)$. Então $q=\left(q_{1}, \ldots, q_{N}\right), \operatorname{com} q_{j}: \mathbb{C}^{N} \rightarrow \mathbb{C}$ para $j=1, \ldots, N$. Observe que as funções $q_{j}$ são da forma

$$
q_{j}(z)=\sum a_{\alpha \beta} z^{\alpha} \bar{z}^{\beta}
$$

com $a_{\alpha \beta} \in \mathbb{C}, \alpha, \beta$ multi-indexados e $z=\left(z_{1}, \ldots, z_{N}\right) \in \mathbb{C}^{N}$.

Para todo $\theta=\left(\theta_{1}, \ldots, \theta_{N}\right) \in \mathbf{S O}(2)^{N} \subset \mathbf{O}(2)$ temos $q(\theta z)=\theta q(z)$ e, portanto,

$$
\left(q_{1}(\theta z), \ldots, q_{N}(\theta z)\right)=\left(e^{i \theta_{1}} q_{1}(z), \ldots, e^{i \theta_{N}} q_{N}(z)\right) .
$$

Logo, $q_{j}(z)=e^{-i \theta_{j}} q_{j}(\theta z)$.

Reescrevendo (3.6) obtemos

$$
q_{j}(z)=\sum a_{\alpha \beta} e^{i\left[\theta_{1}\left(\alpha_{1}-\beta_{1}\right)+\ldots+\theta_{j}\left(\alpha_{j}-\beta_{j}-1\right)+\ldots+\theta_{N}\left(\alpha_{N}-\beta_{N}\right)\right]} z^{\alpha} \bar{z}^{\beta},
$$


para $j=1, \ldots, N$.

Das expressões (3.6) e (3.7) observamos que

$$
\begin{gathered}
a_{\alpha \beta}=0, \text { ou } \\
\alpha_{1}=\beta_{1}, \ldots, \alpha_{j}=\beta_{j}+1, \ldots, \alpha_{N}=\beta_{N} .
\end{gathered}
$$

então,

$$
q_{j}(z)=\sum a_{\beta}\left(z_{1} \overline{z_{1}}\right)^{\beta_{1}} \ldots\left(z_{n} \overline{z_{n}}\right)^{\beta_{N}} z_{j}=\sum a_{\beta}(z \bar{z})^{\beta} z_{j}
$$

Pela equivariância de $q$ em relação a $\kappa_{j}=(I, \ldots, I, \underbrace{\kappa}_{\text {posição j }}, I, \ldots, I)$ segue que

$$
q\left(\kappa_{j} \cdot z\right)=q\left(z_{1}, \ldots, \kappa z_{j}, \ldots, z_{n}\right)=\left(q_{1}(z), \ldots, \kappa q_{j}(z), \ldots, q_{N}(z)\right)=\kappa_{j} \cdot q(z),
$$

ou seja,

$$
q\left(z_{1}, \ldots, \overline{z_{j}}, \ldots, z_{n}\right)=\left(q_{1}(z), \ldots, \overline{q_{j}(z)}, \ldots, q_{N}(z)\right)
$$

implicando em $q_{j}\left(z_{1}, \ldots, \bar{z}_{j}, \ldots, z_{N}\right)=\overline{q_{j}(z)}$.

De (3.8) segue que $\sum a_{\beta}(z \bar{z})^{\beta} \bar{z}_{j}=\sum \bar{a}_{\beta}(z \bar{z})^{\beta} \bar{z}_{j}$ e, portanto, $a_{\beta}=\bar{a}_{\beta}$. Logo $a_{\beta} \in \mathbb{R}$.

Então, $q_{j}(z)=\sum a_{\beta}(z \bar{z})^{\beta} z_{j}$ com $a_{\beta} \in \mathbb{R}$. Como cada $(z \bar{z})^{\beta} \in \mathcal{P}_{\mathbb{C}^{N}}\left(\mathbf{O}(2)^{N}\right)$, o resultado segue.

Lema 3.1.9. (Lema técnico) Seja $h=\left(h_{1}, \ldots, h_{N}\right): \mathbb{R}^{N} \rightarrow \mathbb{R}^{N}$ uma aplicação polinomial, tal que $g(z)=h\left(z_{1} \overline{z_{1}}, \ldots, z_{N} \overline{z_{N}}\right) * z$. Se g é $\boldsymbol{S}_{N^{-}}$-equivariante, então $h$ também é $\boldsymbol{S}_{N^{-} \text {-equivariante. }}$

Demonstração: Se $g$ é $\mathbf{S}_{N^{-}}$equivariante, temos $g(\tau z)=\tau g(z)$ para todo $\tau \in \mathbf{S}_{N}$ e $v \in V$. Mas

$$
g(\tau z)=h(\tau(z * \bar{z})) *(\tau z)=\left(h_{1}(\tau(z * \bar{z})) z_{\tau^{-1}(1)}, \ldots, h_{N}(\tau(z * \bar{z})) z_{\tau^{-1}(N)}\right),
$$

$\mathrm{e}$

$$
\tau g(z)=\left(g_{\tau^{-1}(1)}(z), \ldots, g_{\tau^{-1}(N)}(z)\right)=\left(h_{\tau^{-1}(1)}(z * \bar{z}) z_{\tau^{-1}(1)}, \ldots, h_{\tau^{-1}(N)}(z * \bar{z}) z_{\tau^{-1}(N)}\right) .
$$

Então, para todo $z_{j} \neq 0$, temos $h_{j}(\tau(z * \bar{z}))=h_{\tau^{-1}(j)}(z * \bar{z})$. Assim, obtemos uma relação geral da aplicação polinomial $h_{j}:\left(\mathbb{R}_{+}^{*}\right)^{N} \rightarrow \mathbb{R}$, dada por $h_{j}(\tau(x))=h_{\tau^{-1}(j)}(x)$, para todo $x \in\left(\mathbb{R}_{+}^{*}\right)^{N}$. Como $\left(\mathbb{R}_{+}^{*}\right)^{N}$ é um aberto do $\mathbb{R}^{N}$ e $h_{j}$ é polinomial, ainda temos $h_{j}(\tau(x))=h_{\tau^{-1}(j)}(x)$, para todo $x \in \mathbb{R}^{N}$.

Portanto $h(\tau x)=\tau h(x)$ para todo $x \in \mathbb{R}^{N}$, provando o resultado.

Observe que se $g(z)=h\left(z_{1} \overline{z_{1}}, \ldots, z_{N} \overline{z_{N}}\right) * z i$, o resultado ainda é válido. Tal fato será usado no Teorema 3.2.5.

Teorema 3.1.10. Sejam $g_{1}, \ldots, g_{s}$ geradores para o módulo $\overrightarrow{\mathcal{P}}_{\mathbb{C}^{N}}\left(\boldsymbol{S}_{N}\right)$ sobre o anel $\mathcal{P}_{\mathbb{C}^{N}}\left(\boldsymbol{S}_{N}\right)$. Então, as aplicações

$$
g_{1}\left(z_{1} \overline{z_{1}}, \ldots, z_{N} \overline{z_{N}}\right) * z, \ldots, g_{N}\left(z_{1} \overline{z_{1}}, \ldots, z_{N} \overline{z_{N}}\right) * z
$$

geram o módulo $\overrightarrow{\mathcal{P}}_{\mathbb{C}^{N}}\left(\boldsymbol{O}(2)\right.$ 乙 $\left.\boldsymbol{S}_{N}\right)$ sobre o anel $\mathcal{P}_{\mathbb{C}^{N}}\left(\boldsymbol{O}(2)\right.$ 乙 $\left.\boldsymbol{S}_{N}\right)$. 
Demonstração: Sejam $g_{1}, \ldots, g_{N}$ geradores de $\overrightarrow{\mathcal{P}}_{\mathbb{C}^{N}}\left(\mathbf{S}_{N}\right)$ e seja $q \in \overrightarrow{\mathcal{P}}_{\mathbb{C}^{N}}\left(\mathbf{O}(2)\right.$ 乙 $\left.\mathbf{S}_{N}\right)$. Em particular, $q$ é equivariante pelas ações de $\mathbf{O}(2)^{N}$ e de $\mathbf{S}_{N}$. Então existem $h_{i}: \mathbb{R}^{N} \rightarrow$ $\mathbb{R}$ para $i=1 \ldots, N$, tais que

$$
q(z)=\left(h_{1}\left(z_{1} \bar{z}_{1}, \ldots, z_{N} \bar{z}_{N}\right) z_{1}, \ldots, h_{N}\left(z_{1} \bar{z}_{1}, \ldots, z_{N} \bar{z}_{N}\right) z_{N}\right)=h\left(z_{1} \bar{z}_{1}, \ldots, z_{N} \bar{z}_{N}\right) * z,
$$

onde $z=\left(z_{1}, \ldots, z_{N}\right) \in \mathbb{C}^{N}$ e $h=\left(h_{1}, \ldots, h_{n}\right)$.

Pelo Lema 3.1.9, segue que $h$ também é $\mathbf{S}_{N^{-}}$equivariante. Portanto $h=\sum_{i=1}^{s} p_{i} g_{i}$ com $p_{i} \in \mathcal{P}_{\mathbb{C}^{N}}\left(\mathbf{S}_{N}\right)$, para $i=1, \ldots, s$.

Logo,

$$
\begin{aligned}
q(z) & =\left(\sum_{i=1}^{s} p_{i}\left(z_{1} \overline{z_{1}}, \ldots, z_{N} \overline{z_{N}}\right) g_{i}\left(z_{1} \overline{z_{1}}, \ldots, z_{N} \overline{z_{N}}\right)\right) * z \\
& =\sum_{i=1}^{s} p_{i}\left(z_{1} \overline{z_{1}}, \ldots, z_{N} \overline{z_{N}}\right)\left[g_{i}\left(z_{1} \overline{z_{1}}, \ldots, z_{N} \overline{z_{N}}\right) * z\right],
\end{aligned}
$$

mostrando o resultado.

Corolário 3.1.11. Os elementos da forma

$$
\left(z_{1} \bar{z}_{1}\right)^{k}+\left(z_{2} \bar{z}_{2}\right)^{k}+\ldots+\left(z_{N} \bar{z}_{N}\right)^{k}, \text { para } k=1, \ldots, N-1
$$

formam uma base de Hilbert para o anel $\mathcal{P}_{\mathbb{C}^{N}}\left(\boldsymbol{O}(2)\right.$ 乙 $\left.\boldsymbol{S}_{N}\right)$.

Demonstração: Segue diretamente do Lema 3.1.3 e do Teorema 3.1.7.

Corolário 3.1.12. O módulo $\overrightarrow{\mathcal{P}}_{\mathbb{C}^{N}}\left(\boldsymbol{O}(2) \curlywedge \boldsymbol{S}_{N}\right)$ é gerado sobre o anel $\mathcal{P}_{\mathbb{C}^{N}}\left(\boldsymbol{O}(2) \curlywedge \boldsymbol{S}_{N}\right)$ pelas aplicaçôes

$$
\left(\begin{array}{c}
z_{1}\left(z_{1} \bar{z}_{1}\right)^{k} \\
z_{2}\left(z_{2} \bar{z}_{2}\right)^{k} \\
\vdots \\
z_{N}\left(z_{N} \bar{z}_{N}\right)^{k}
\end{array}\right)
$$

para $k=0, \ldots, N-1$.

Demonstração: Segue direto do Lema 3.1.4 e do Teorema 3.1.10.

\subsection{O produto coroa $\Gamma$ ? $\mathrm{S}_{N}$ como grupo de simetrias e anti-simetrias}

Quando um sistema sob a ação do produto coroa não possui anti-simetrias em $\Gamma$, dizemos que esse sistema é puramente equivariante. Na seção anterior estudamos os 
casos puramente equivariantes. Nesta seção, apresentamos o problema de acoplamento de células do grupo produto coroa $\Gamma$ < $\mathbf{S}_{N}$, na presença de anti-simetrias em $\Gamma$.

Começamos definindo um homomorfismo entre $\Gamma^{N}$ e $\mathbf{Z}_{2}^{N}$. Considere o homomorfismo $\sigma: \Gamma \rightarrow \mathbf{Z}_{2}$ de índice 2, definido em (2.1). Definimos o homomorfismo induzido $\tilde{\sigma}$ como

$$
\begin{aligned}
\tilde{\sigma}: \Gamma^{N} & \rightarrow \mathbf{Z}_{2}^{N} \\
\gamma & \mapsto\left(\sigma\left(\gamma_{1}\right), \ldots, \sigma\left(\gamma_{N}\right)\right),
\end{aligned}
$$

para $\gamma=\left(\gamma_{1}, \ldots, \gamma_{N}\right) \in \Gamma^{N}$

A partir daí, definimos a ação dual induzida de $\Gamma^{N}$ em $V^{N}$ da ação definida em 3.1, dada pela ação diagonal

$$
\gamma \cdot v=\left(\sigma\left(\gamma_{1}\right) \gamma_{1} v_{1}, \ldots, \sigma\left(\gamma_{N}\right) \gamma_{N} v_{N}\right)=\tilde{\sigma}(\gamma) *(\gamma v)
$$

e a ação de $\Gamma$ ใ $\mathbf{S}_{N}$ em $V^{N}$ dada por

$$
(\gamma, \tau) \odot v=\left(\sigma\left(\gamma_{1}\right) \gamma_{1} v_{\tau^{-1}(1)}, \ldots, \sigma\left(\gamma_{N}\right) \gamma_{N} v_{\tau^{-1}(N)}\right)=\tilde{\sigma}(\gamma) *((\gamma, \tau) v)
$$

para todo $\gamma=\left(\gamma_{1}, \ldots, \gamma_{N}\right) \in \Gamma^{N}, \tau \in \mathbf{S}_{N}$ e $v=\left(v_{1}, \ldots, v_{N}\right) \in V^{N}$. Esta é chamada ação dual induzida em $V^{N}$ da ação definida em (3.3).

Aqui, a operação $*$ representa o produto das componentes de mesmo índice de dois vetores, como introduzido na Seção 3.1.

Observe que de fato $\odot$ é uma ação, pois como

$$
(\gamma, \tau) \odot[(\delta, \varrho) \odot v]=(\gamma, \tau) \odot\left(\sigma\left(\delta_{1}\right) \delta_{1} v_{\varrho^{-1}(1)}, \ldots, \sigma\left(\delta_{N}\right) \delta_{N} v_{\varrho^{-1}(N)}\right),
$$

tomamos $y_{i}=\sigma\left(\delta_{i}\right) \delta_{i} v_{\varrho^{-1}(i)}$ e obtemos

$$
\begin{aligned}
(\gamma, \tau) \odot[(\delta, \varrho) \odot v]= & (\gamma, \tau) \odot\left(y_{1}, \ldots, y_{N}\right) \\
= & \left(\sigma\left(\gamma_{1}\right) \gamma_{1} y_{\varrho^{-1}(1)}, \ldots, \sigma\left(\gamma_{N}\right) \gamma_{N} y_{\varrho^{-1}(N)}\right) \\
= & \left(\sigma\left(\gamma_{1}\right) \gamma_{1} \sigma\left(\delta_{\tau^{-1}(1)}\right) \delta_{\tau^{-1}(1)} v_{\varrho^{-1}\left(\varrho^{-1}(1)\right)},\right. \\
& \ldots, \sigma\left(\gamma_{N}\right) \gamma_{N} \sigma\left(\delta_{\tau^{-1}(N)}\right) \delta_{\tau^{-1}(N)} v_{\varrho^{-1}\left(\varrho^{-1}(N)\right)} \\
= & \left(\sigma\left(\gamma_{1} \delta_{\tau^{-1}(1)}\right) \gamma_{1} \delta_{\tau^{-1}(1)} v_{(\tau \varrho)^{-1}(1)},\right. \\
& \left.\ldots, \sigma\left(\gamma_{N} \delta_{\tau^{-1}(N)}\right) \gamma_{N} \delta_{\tau^{-1}(N)} v_{(\tau \varrho)^{-1}(N)}\right) \\
= & {[(\gamma, \tau)(\delta, \varrho)] \odot v . }
\end{aligned}
$$

Definição 3.2.1. Um germe de aplicação $g: V^{N} \rightarrow V^{N}$ é chamado $\Gamma^{N}$-reversívelequivariante se

$$
g(\gamma x)=\gamma \cdot g(x)=\tilde{\sigma}(\gamma) *(\gamma g(x)),
$$

onde . denota a ação definida em (3.9).

Denotamos o espaço de todas as aplicações polinomiais $\Gamma^{N}$-reversíveis-equivariantes por $\overrightarrow{\mathcal{Q}}\left(\Gamma^{N}\right)$. Observe que $\overrightarrow{\mathcal{Q}}\left(\Gamma^{N}\right)$ tem estrutura de módulo sobre o anel $\mathcal{P}_{V^{N}}\left(\Gamma^{N}\right)$.

Definição 3.2.2. Um germe de aplicação $g: V^{N} \rightarrow V^{N}$ é chamado $\Gamma$ ? $\boldsymbol{S}_{N}$-reversívelequivariante se

$$
g((\gamma, \tau) x)=(\gamma, \tau) \odot g(x)=\tilde{\sigma}(\gamma) *((\gamma, \tau) g(x)),
$$

onde $\odot$ denota a ação definida em 3.10 .

Denotamos o espaço de todas as aplicações polinomiais $\Gamma$ $2 \mathbf{S}_{N}$-reversíveis-equivariantes por $\overrightarrow{\mathcal{Q}}\left(\Gamma \succ \mathbf{S}_{N}\right)$. Note que $\overrightarrow{\mathcal{Q}}\left(\Gamma \imath \mathbf{S}_{N}\right)$ tem estrutura de módulo sobre o anel $\mathcal{P}_{V^{N}}\left(\Gamma \succ \mathbf{S}_{N}\right)$. 


\subsubsection{Geradores dos reversíveis-equivariantes para o grupo $\mathrm{O}(2)$ 々 $\mathbf{S}_{N}$}

Consideramos aqui o homomorfismo $\sigma: \mathbf{O}(2)=\langle\mathbf{S O}(2), \kappa\rangle \rightarrow \mathbf{Z}_{2}$ tal que $\kappa$ é uma anti-simetria, isto é, $\sigma(\kappa)=-1$ e $\sigma(\theta)=1, \forall \theta \in \mathbf{S O}(2)$.

Teorema 3.2.3. Considere as aplicações

$$
g_{1}(z)=\left(z_{1} i, 0, \ldots, 0\right), \ldots, g_{N}(z)=\left(0, \ldots, 0, z_{N} i\right)
$$

Então, o conjunto $\left\{g_{1}, \ldots, g_{N}\right\}$ gera o módulo $\overrightarrow{\mathcal{Q}}\left(\boldsymbol{O}(2)^{N}\right)$ sobre o anel $\mathcal{P}_{\mathbb{C}^{N}}\left(\boldsymbol{O}(2)^{N}\right)$.

Demonstração: Seja $q: \mathbb{C}^{N} \rightarrow \mathbb{C}^{N}$ tal que $q \in \overrightarrow{\mathcal{Q}}\left(\mathbf{O}(2)^{N}\right)$. Então $q=\left(q_{1}, \ldots, q_{N}\right)$ com $q_{j}: \mathbb{C}^{N} \rightarrow \mathbb{C}$ onde

$$
q_{j}(z)=\sum a_{\alpha \beta} z_{1}^{\alpha_{1}}{\overline{z_{1}}}^{\beta_{1}} \ldots z_{N}^{\alpha_{N}} \overline{z_{N} \beta_{N}}=\sum a_{\alpha \beta} z^{\alpha} \bar{z}^{\beta}
$$

para $\alpha$ e $\beta$ multi-índices e $z=\left(z_{1}, \ldots, z_{N}\right) \in \mathbb{C}^{N}$.

Para todo $\theta=\left(\theta_{1}, \ldots, \theta_{N}\right) \in \mathbf{S O}(2)^{N} \subset \mathbf{O}(2)$ temos $q(\theta z)=\tilde{\sigma}(\theta) * \theta q(z)$ e, portanto, $\left(q_{1}(\theta z), \ldots, q_{N}(\theta z)\right)=\left(\sigma\left(\theta_{1}\right) e^{i \theta_{1}} q_{1}(z), \ldots, \sigma\left(\theta_{N}\right) e^{i \theta_{N}} q_{N}(z)\right)=\left(e^{i \theta_{1}} q_{1}(z), \ldots, e^{i \theta_{N}} q_{N}(z)\right)$. Logo, $q_{j}(\theta z)=e^{i \theta_{j}} q_{j}(z)$. Pelo mesmo argumento que usamos em (3.7) obtemos

$$
q_{j}(z)=\sum a_{\beta}\left(z_{1} \overline{z_{1}}\right)^{\beta_{1}} \ldots\left(z_{N} \overline{z_{N}}\right)^{\beta_{N}} z_{j}=\sum a_{\beta}(z \bar{z})^{\beta} z_{j},
$$

com $a_{\beta} \in \mathbb{C}$. Mostremos agora, que $a_{\beta} \in \mathbb{C}$ é puramente imaginário.

Pela equivariância de $q$ em relação a $\kappa_{j}=(I, \ldots, I, \underbrace{\kappa}_{\text {posição } \mathrm{j}}, I, \ldots, I)$ segue que $q\left(\kappa_{j} z\right)=\tilde{\sigma}\left(\kappa_{j}\right) * \kappa_{j} q(z)$, isto é,

$$
q\left(\kappa_{j} z\right)=q\left(\sigma(1) z_{1}, \ldots, \sigma(\kappa) \kappa z_{j}, \ldots, \sigma(1) z_{n}\right),
$$

e portanto,

$$
q\left(z_{1}, \ldots, \overline{z_{j}}, \ldots, z_{n}\right)=\left(q_{1}(z), \ldots,-\overline{q_{j}(z)}, \ldots, q_{N}(z)\right)
$$

implicando em, $q_{j}\left(\kappa_{j} z\right)=-\overline{q_{j}(z)}$. De (3.11) obtemos $a_{\beta}=-\bar{a}_{\beta}$, mostrando que $a_{\beta}$ é puramente imaginário. Então $a_{\beta}=b_{\beta} i, \operatorname{com} b_{\beta} \in \mathbb{R}$.

Portanto, $q_{j}(z)=\sum b_{\beta}(z \bar{z})^{\beta} z_{j} i \operatorname{com} b_{\beta} \in \mathbb{R}$. Como cada $(z \bar{z})^{\beta} \in \mathcal{P}_{\mathbb{C}^{N}}\left(\mathbf{O}(2)^{N}\right)$, segue o resultado.

Lema 3.2.4. Se $g: V^{N} \rightarrow V^{N}$ é $\Gamma$ 々 $\boldsymbol{S}_{N^{-}}$-reversível-equivariante, então g também é reversível-equivariante pela ação de $\Gamma^{N}$ em $V^{N}$ e equivariante pela ação de $\boldsymbol{S}_{N}$ em $V^{N}$.

Demonstração: Se $q \in \overrightarrow{\mathcal{Q}}\left(\Gamma \imath \mathbf{S}_{N}\right)$, temos $q((\gamma, \tau) v)=(\gamma, \tau) \odot q(v)=\tilde{\sigma}(\gamma) *(\gamma, \tau) q(v)$ para todo $v \in V^{N}$.

Identificamos o grupo $\Gamma^{N}$ com um subgrupo do produto coroa $\Gamma$ 々 $\mathbf{S}_{N}$, associando um elemento $\gamma \in \Gamma^{N}$ ao elemento $(\gamma, I) \in \Gamma$ 々 $\mathbf{S}_{N}$. 
Então

$$
q(\gamma v)=q((\gamma, I) v)=\tilde{\sigma}(\gamma) *(\gamma, I) q(v)=\tilde{\sigma}(\gamma) * \gamma q(v)=\gamma \cdot q(v)
$$

Segue que $q$ é $\Gamma^{N}$-reversível-equivariante.

Analogamente associamos um elemento $\tau \in \mathbf{S}_{N}$ ao elemento $(I, \tau) \in \Gamma$ 々 $\mathbf{S}_{N}$. Então

$$
q(\tau v)=q((I, \tau) v)=\tilde{\sigma}(I) *(I, \tau) q(v)=(I, \tau) q(v)=\tau q(v) .
$$

Logo $q \in \overrightarrow{\mathcal{P}}_{V^{N}}\left(\mathbf{S}_{N}\right)$

Teorema 3.2.5. Sejam $g_{1}, \ldots, g_{s}$ geradores para o módulo $\overrightarrow{\mathcal{P}}_{\mathbb{C}^{N}}\left(\boldsymbol{S}_{N}\right)$ sobre o anel $\mathcal{P}_{\mathbb{C}^{N}}\left(\boldsymbol{S}_{N}\right)$. Então, as aplicações

$$
g_{1}\left(z_{1} \overline{z_{1}}, \ldots, z_{N} \overline{z_{N}}\right) * z i, \ldots, g_{N}\left(z_{1} \overline{z_{1}}, \ldots, z_{N} \overline{z_{N}}\right) * z i
$$

geram o módulo $\overrightarrow{\mathcal{Q}}\left(\boldsymbol{O}(2)\right.$ 乙 $\left.\boldsymbol{S}_{N}\right)$ sobre o anel $\mathcal{P}_{\mathbb{C}^{N}}\left(\boldsymbol{O}(2)\right.$ 乙 $\left.\boldsymbol{S}_{N}\right)$.

Demonstração: Seja $q \in \overrightarrow{\mathcal{Q}}\left(\mathbf{O}(2) \imath \mathbf{S}_{N}\right)$. Pelo Lema 3.2.4 anterior, segue que $q$ também é $\mathbf{O}(2)^{N}$-reversível-equivariante. Segue do Teorema 3.2 .3 que existem funções $h_{i}: \mathbb{R}^{N} \rightarrow$ $\mathbb{R}$, para $i=1, \ldots, N$, tais que

$$
q(z)=h_{1}\left(z_{1} \bar{z}_{1}, \ldots, z_{N} \bar{z}_{N}\right)\left(z_{1} i, 0, \ldots, 0\right)+\ldots+h_{N}\left(z_{1} \bar{z}_{1}, \ldots, z_{N} \bar{z}_{N}\right)\left(0, \ldots, z_{N} i\right) .
$$

Tomando $h=\left(h_{1}, \ldots, h_{N}\right)$ escrevemos

$$
q(z)=h\left(z_{1} \bar{z}_{1}, \ldots, z_{N} \bar{z}_{N}\right) * z i
$$

Pelo Lema 3.2.4 acima, $q$ também é $\mathbf{S}_{N}$-equivariante e, portanto, segue do Lema técnico 3.1.9 que $h$ também é $\mathbf{S}_{N^{-}}$equivariante. Logo, $h=\sum_{j=1}^{s} p_{j} g_{j}$ onde $p_{j} \in \mathcal{P}_{\mathbb{C}^{N}}\left(\mathbf{S}_{N}\right)$ para $j=1, \ldots, s$.

Portanto

$$
q(z)=\sum_{j=1}^{s} p_{j}\left(z_{1} \bar{z}_{1}, \ldots, z_{N} \bar{z}_{N}\right)\left(g_{j}\left(z_{1} \bar{z}_{1}, \ldots, z_{N} \bar{z}_{N}\right) * z i\right),
$$

mostrando o resultado.

Corolário 3.2.6. O módulo $\overrightarrow{\mathcal{Q}}\left(\boldsymbol{O}(2) \backslash \boldsymbol{S}_{N}\right)$ é gerado sobre o anel $\mathcal{P}\left(\boldsymbol{O}(2) \backslash \boldsymbol{S}_{N}\right)$ pelas aplicações

$$
\left(\begin{array}{c}
\left(z_{1} \bar{z}_{1}\right)^{k} z_{1} i \\
\left(z_{2} \bar{z}_{2}\right)^{k} z_{2} i \\
\vdots \\
\left(z_{N} \bar{z}_{N}\right)^{k} z_{N} i
\end{array}\right)
$$

para $k=0, \ldots, N-1$.

Demonstração: Segue diretamente do Lema 3.1.4 e do Teorema 3.2.5 acima. 



\section{Capítulo 4}

\section{Exemplos}

Neste Capítulo exibimos as formas gerais de aplicações reversíveis-equivariantes sob a ação de diferentes grupos, utilizando os algoritmos estudados no Capítulo 2.

Como sempe denotamos por $\Gamma_{+}$o grupo das simetrias e por $\Gamma_{-}$o conjunto das antisimetrias, de modo que, fixando uma anti-simetria $\delta \in \Gamma_{-}$arbitrária temos $\Gamma=\Gamma_{+} \dot{\cup} \delta \Gamma_{+}$.

Em alguns momentos o cálculo para uma base de Hilbert para o anel dos polinômios invariantes é algo simples. Quando isso não ocorre, recorremos ao programa Singular, o qual nos fornece uma base de Hilbert para o anel. Em casos em que precisamos encontrar um conjunto de geradores para o módulo $\overrightarrow{\mathcal{P}}_{V, W}\left(\Gamma_{+}\right)$, utilizamos a técnica apresentada na demonstração do Teorema 1.3.18. A idéia é induzir a ação de $\Gamma_{+}$em $V \times W$, dada por $\gamma \cdot(x, y)=\left(\rho_{\gamma} x, \eta_{\gamma} y\right)$, e encontrar uma base de Hilbert $\left\{u_{1}, \ldots, u_{s}\right\}$ para o anel $\mathcal{P}_{V \times W}\left(\Gamma_{+}\right)$. Em seguida calculamos a derivada $\left(d_{y} u_{i}\right)_{(x, 0)}$, para $x \in V$ e $y, 0 \in W$. $\mathrm{O}$ Teorema nos diz que o conjunto $\left\{\left(d_{y} u_{1}\right)_{(x, 0)}^{t}, \ldots,\left(d_{y} u_{s}\right)_{(x, 0)}^{t}\right\}$ gera o módulo $\overrightarrow{\mathcal{P}}_{V, W}\left(\Gamma_{+}\right)$ sobre o anel $\mathcal{P}_{V}\left(\Gamma_{+}\right)$.

São 11 exemplos apresentados aqui, organizados em cinco etapas. A primeira etapa é obtida com o cálculo manual nos exemplos simples ou com o uso do programa Singular [21] nos demais. Em alguns exemplos, ilustramos retratos de fase de campos vetoriais reversíveis-equivariantes. A regra de coloração em cada figura é da seguinte forma: fixamos uma trajetória em preto e colorimos de verde à sua simétrica e de vermelho à sua anti-simétrica. O comportamento qualitativo das trajetórias foi obtida por Baptistelli $[5]$.

\subsection{Forma geral $\mathrm{Z}_{2}$-reversível-equivariante}

Considere a ação padrão do grupo $\mathbf{Z}_{2}=\left\{R_{\pi}, I\right\}$ em $\mathbb{R}^{2}$, onde

$$
R_{\pi}=\left(\begin{array}{cc}
-1 & 0 \\
0 & -1
\end{array}\right)
$$

1. Obtemos manualmente os elementos $u_{1}(x, y)=x$ e $u_{2}(x, y)=y$ para uma base de Hilbert para o anel $\mathcal{P}\left(\Gamma_{+}\right)$.

2. Se $q \in \overrightarrow{\mathcal{P}}\left(\Gamma_{+}\right)$, então $q(x, y)=\left(q_{1}(x, y), q_{2}(x, y)\right)=q_{1}(x, y)(1,0)+q_{2}(x, y)(0,1)$ com $q_{i} \in \mathcal{P}\left(\Gamma_{+}\right)$, para $i=1,2$. Como $H_{0}(x, y)=(1,0)$ e $H_{1}(x, y)=(0,1)$ são 
$\Gamma_{+}$-equivariante. Então, o conjunto $\left\{H_{0}, H_{1}\right\}$ gera o módulo $\overrightarrow{\mathcal{P}}\left(\Gamma_{+}\right)$sobre o anel $\mathcal{P}\left(\Gamma_{+}\right)$.

3. Definimos $\tilde{u}_{0}(x, y)=1$ e pelo Algorítmo 2.3 .10 obtemos $\tilde{u}_{1}(x, y)=x$ e $\tilde{u}_{2}(x, y)=y$.

4. Calculamos os geradores para o módulo $\overrightarrow{\mathcal{P}}\left(\Gamma_{+}\right)$sobre o anel $\mathcal{P}\left(\mathbf{Z}_{2}\right)$ :

$$
\begin{aligned}
& H_{00}(x, y)=H_{0}(x, y)=(1,0) ; \\
& H_{01}(x, y)=H_{1}(x, y)=(0,1) ; \\
& H_{10}(x, y)=\tilde{u}_{1}(x, y) H_{0}(x, y)=(x, 0) ; \\
& H_{11}(x, y)=\tilde{u}_{1}(x, y) H_{1}(x, y)=(0, x) ; \\
& H_{20}(x, y)=\tilde{u}_{2}(x, y) H_{0}(x, y)=(y, 0) ; \\
& H_{21}(x, y)=\tilde{u}_{2}(x, y) H_{1}(x, y)=(0, y) .
\end{aligned}
$$

5. Por fim, com o Algoritmo 2.3.13 vamos obter os geradores para o módulo dos reversíveis-equivariantes.

Temos $\tilde{H}_{10} \equiv \tilde{H}_{11} \equiv \tilde{H}_{20} \equiv \tilde{H}_{21} \equiv 0$. Note ainda, que $\delta^{-1} H_{00}(\delta(x, y))=-H_{00}$ e $\delta^{-1} H_{01}(\delta(x, y))=-H_{01}$, e portanto,

$$
\tilde{H}_{00}(x, y)=(1,0) \quad \text { e } \quad \tilde{H}_{01}(x, y)=(0,1)
$$

Portanto o conjunto $\{(1,0),(0,1)\}$ gera o módulo $\overrightarrow{\mathcal{Q}}\left(\mathbf{Z}_{2}\right)$ sobre o anel $\mathcal{P}\left(\mathbf{Z}_{2}\right)$.

Observação 4.1.1. Nesse exemplo, temos $\operatorname{Fix}\left(\boldsymbol{Z}_{2}\right)=\{0\}$ e portanto pela Proposição 1.4.10, todo germe de aplicação $f: \mathbb{R}^{2} \rightarrow \mathbb{R}^{2} \Gamma$-equivariante satisfaz $f(0)=0$, e o único germe de função linear $\Gamma$-invariante é a função nula.

\subsection{Forma geral $\mathrm{Z}_{4}$-reversível-equivariante}

Considere a ação padrão do grupo $\mathbf{Z}_{4}$ em $\mathbb{R}^{2}$ dada pela multiplicação de matrizes. Fixemos $\delta=R_{\frac{\pi}{2}}$ como uma anti-simetria e, então, $\Gamma_{+}=\mathbf{Z}_{2}$, o qual é gerado pela rotação $R_{\pi}$.

1. Com um cálculo direto obtemos uma base para $\mathcal{P}\left(\mathbf{Z}_{2}\right)$ :

$$
u_{1}(x, y)=x^{2}, \quad u_{2}(x, y)=y^{2} \quad \text { e } \quad u_{3}(x, y)=x y .
$$

2. Com a técnica do Teorema 1.3.18 encontramos geradores para $\overrightarrow{\mathcal{P}}\left(\mathbf{Z}_{2}\right)$ sobre o anel $\overrightarrow{\mathcal{P}}\left(\mathbf{Z}_{2}\right)$ :

$$
\begin{aligned}
& H_{0}(x, y)=(x, 0) \\
& H_{1}(x, y)=(y, 0) \\
& H_{2}(x, y)=(0, x) \\
& H_{3}(x, y)=(0, y)
\end{aligned}
$$


3. Definimos $\tilde{u}_{0} \equiv 1$. Do Algoritmo 2.3.10 resulta:

$$
\begin{aligned}
& \tilde{u}_{1}(x, y)=x^{2}-y^{2} \\
& \tilde{u}_{2}(x, y)=y^{2}-x^{2}=-\tilde{u}_{1}(x, y) ; \\
& \tilde{u}_{3}(x, y)=x y .
\end{aligned}
$$

4. Calculamos os geradores para o módulo $\overrightarrow{\mathcal{P}}\left(\mathbf{Z}_{2}\right)$ sobre o anel $\mathcal{P}\left(\mathbf{Z}_{4}\right)$ :

$$
\begin{aligned}
& H_{00}(x, y)=H_{0}(x, y)=(x, 0) ; \\
& H_{01}(x, y)=H_{1}(x, y)=(y, 0) ; \\
& H_{02}(x, y)=H_{2}(x, y)=(0, x) ; \\
& H_{03}(x, y)=H_{3}(x, y)=(0, y) ; \\
& H_{10}(x, y)=\tilde{u}_{1}(x, y) H_{0}(x, y)=\left(x^{3}-x y^{2}, 0\right) ; \\
& H_{11}(x, y)=\tilde{u}_{1}(x, y) H_{1}(x, y)=\left(x^{2} y-y^{3}, 0\right) ; \\
& H_{12}(x, y)=\tilde{u}_{1}(x, y) H_{2}(x, y)=\left(0, x^{3}-x y^{2}\right) ; \\
& H_{13}(x, y)=\tilde{u}_{1}(x, y) H_{3}(x, y)=\left(0, x^{2} y-y^{3}\right) ; \\
& H_{30}(x, y)=\tilde{u}_{3}(x, y) H_{0}(x, y)=\left(x^{2} y, 0\right) ; \\
& H_{31}(x, y)=\tilde{u}_{3}(x, y) H_{1}(x, y)=\left(x y^{2}, 0\right) ; \\
& H_{32}(x, y)=\tilde{u}_{3}(x, y) H_{2}(x, y)=\left(0, x^{2} y\right) ; \\
& H_{33}(x, y)=\tilde{u}_{3}(x, y) H_{3}(x, y)=\left(0, x y^{2}\right) ;
\end{aligned}
$$

5. Com o Algoritmo 2.3.13, encontramos os seguintes geradores para o módulo $\overrightarrow{\mathcal{Q}}\left(\mathbf{Z}_{4}\right)$ :

$$
\begin{aligned}
& \tilde{H}_{00}(x, y)=-\tilde{H}_{03}(x, y)=(x,-y) ; \\
& \tilde{H}_{01}(x, y)=\tilde{H}_{02}(x, y)=(y, x) ; \\
& \tilde{H}_{10}(x, y)=\tilde{H}_{13}(x, y)=\left(x^{3}-x y^{2}, x^{2} y-y^{3}\right) ; \\
& \tilde{H}_{11}(x, y)=-\tilde{H}_{12}(x, y)=\left(x^{2} y-y^{3}, x y^{2}-x^{3}\right) ; \\
& \tilde{H}_{30}(x, y)=\tilde{H}_{33}(x, y)=\left(x^{2} y, x y^{2}\right) ; \\
& \tilde{H}_{31}(x, y)=-\tilde{H}_{32}(x, y)=\left(x y^{2},-x^{2} y\right) .
\end{aligned}
$$

Observe nas Figuras 4.1 e 4.2, exemplos de campos vetoriais $\mathbf{Z}_{4}$-reversívei-equivariantes, para

$$
\dot{x}=g(x),
$$

onde

$$
g(x, y)=\left(x-3 x^{4} y-2 x^{2} y^{3}+y^{5},-y+x^{5}-2 x^{3} y^{2}-3 x y^{4}\right),
$$

na Figura $4.1 \mathrm{e}$

$$
g(x, y)=\left(y-3 x^{2} y+y^{3}, x+x^{3}-3 x y^{2}\right)
$$

na Figura 4.2. 


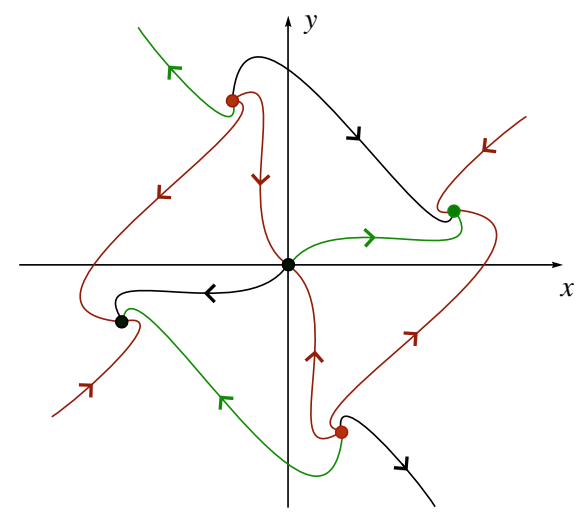

Figura 4.1: Campo vetorial $\mathbf{Z}_{4}$-reversível-equivariante dado em 4.1.

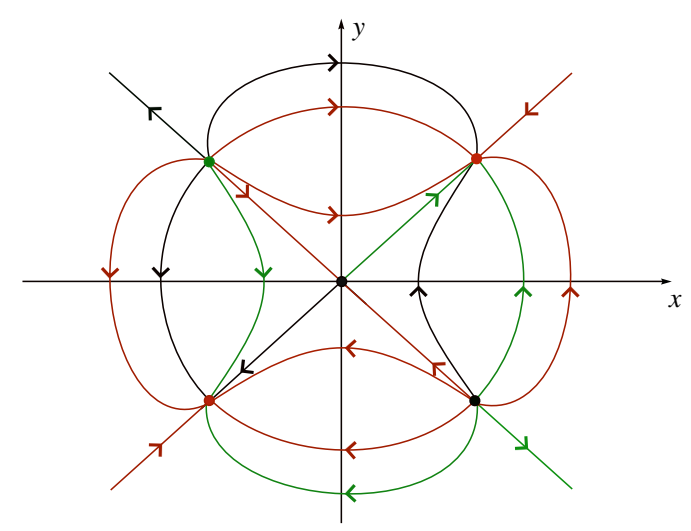

Figura 4.2: Campo vetorial $\mathbf{Z}_{4}$-reversível-equivariante dado em 4.2.

\subsection{Forma geral $\mathrm{Z}_{2} \oplus \mathrm{Z}_{2}$-reversível-equivariante}

Considere a ação padrão de $\Gamma=\mathbf{Z}_{2} \oplus \mathbf{Z}_{2}=\left\langle\kappa_{1}, \kappa_{2}\right\rangle$ no plano onde

$$
\kappa_{1}=\left(\begin{array}{cc}
1 & 0 \\
0 & -1
\end{array}\right) \text { e } \kappa_{2}=\left(\begin{array}{cc}
-1 & 0 \\
0 & 1
\end{array}\right)
$$

Fixamos $\delta=\kappa_{1}$ como uma anti-simetria. Neste caso temos $\Gamma_{+}=\left\{I, \kappa_{2}\right\}$.

1. Observe que a ação de $\kappa_{2} \in \Gamma_{+}$num elemento $(x, y) \in \mathbb{R}^{2}$ fixa a coordenada $y$ e muda o sinal da coordenada $x$. Então um polinômio $p: \mathbb{R}^{2} \rightarrow \mathbb{R}$ é $\Gamma_{+}$-invariante se $p(x, y)=\sum a_{i j}\left(x^{2}\right)^{i} y^{j}$. Ou seja, as funções

$$
u_{1}(x, y)=x^{2} \quad \text { e } \quad u_{2}(x, y)=y
$$

formam uma base de Hilbert para o anel $\mathcal{P}\left(\Gamma_{+}\right)$. 
2. Seja $q \in \overrightarrow{\mathcal{P}}\left(\Gamma_{+}\right)$, então $q(x, y)=\left(q_{1}(x, y), q_{2}(x, y)\right)$. Como $q\left(\kappa_{2}(x, y)\right)=\kappa_{2} q(x, y)$ segue que

$$
q_{1}(-x, y)=-q_{1}(x, y) \quad \text { e } \quad q_{2}(-x, y)=q_{1}(x, y)
$$

notando que $q_{2} \in \mathcal{P}\left(\Gamma_{+}\right)$.

Escrevendo $q_{1}$ na forma $q_{1}(x, y)=\sum a_{i j} x^{i} y^{j}$, obtemos $\sum a_{i j}(-x)^{i} y^{j}=-\sum a_{i j} x^{i} y^{j}$, e portanto, $(-1)^{i}=a_{i j}=-a_{i j}$. Logo, $a_{i j}=0$ ou $i$ é ímpar, ou seja, $i=2 k+1$. Então $q_{1}(x, y)=\left(\sum a_{k j}\left(x^{2}\right)^{k} y^{j}\right) x$, onde $q_{1}^{\prime}(x, y)=\sum a_{k j}\left(x^{2}\right)^{k} y^{j} \in \mathcal{P}\left(\Gamma_{+}\right)$pelo item 1.

$\operatorname{Logo} q(x, y)=q_{1}^{\prime}(x, y)(x, 0)+q_{2}(x, y)(0,1)$, onde $q_{1}^{\prime}, q_{2} \in \mathcal{P}\left(\Gamma_{+}\right)$. Tomando $H_{0}(x, y)=(x, 0)$ e $H_{1}(x, y)=(0,1)$ temos $H_{0}, H_{1} \in \overrightarrow{\mathcal{P}}\left(\Gamma_{+}\right)$.

Portanto o conjunto $\left\{H_{0}, H_{1}\right\}$ gera o módulo $\overrightarrow{\mathcal{P}}\left(\Gamma_{+}\right)$sobre o anel $\mathcal{P}\left(\Gamma_{+}\right)$.

3. Definimos $\tilde{u}_{0}(x, y)=1$. Pelo Algoritmo 2.3.10 obtemos $\tilde{u}_{1}(x, y)=0$ e $\tilde{u}_{2}(x, y)=y$.

4. Calculamos os geradores para o módulo $\overrightarrow{\mathcal{P}}\left(\Gamma_{+}\right)$sobre o anel $\mathcal{P}\left(\mathbf{Z}_{2} \oplus \mathbf{Z}_{2}\right)$ :

$$
\begin{aligned}
& H_{00}(x, y)=H_{0}(x, y)=(x, 0) \\
& H_{01}(x, y)=H_{1}(x, y)=(0,1) ; \\
& H_{10}(x, y)=\tilde{u}_{1}(x, y) H_{0}(x, y)=(0,0) ; \\
& H_{11}(x, y)=\tilde{u}_{1}(x, y) H_{1}(x, y)=(0,0) ; \\
& H_{20}(x, y)=\tilde{u}_{2}(x, y) H_{0}(x, y)=(x y, 0) ; \\
& H_{21}(x, y)=\tilde{u}_{2}(x, y) H_{1}(x, y)=(0, y) .
\end{aligned}
$$

5. Pelo Algoritmo 2.3.13 obtemos os seguintes geradores para $\overrightarrow{\mathcal{Q}}\left(\mathbf{Z}_{2} \oplus \mathbf{Z}_{2}\right)$ como módulo sobre o anel $\mathcal{P}\left(\mathbf{Z}_{2} \oplus \mathbf{Z}_{2}\right)$ :

$$
\begin{aligned}
& \tilde{H}_{01}(x, y)=(0,1) \\
& \tilde{H}_{20}(x, y)=(x y, 0) .
\end{aligned}
$$

\subsection{Forma geral $\mathrm{O}(2)$-reversível-equivariante}

Considere agora a ação do grupo $\mathbf{O}(2)$ em $\mathbb{C} \times \mathbb{R}$ dada por

$$
\theta(z, x)=\left(e^{i \theta} z, x\right) \quad \text { e } \quad \kappa(z, x)=(\bar{z},-x),
$$

onde $\theta \in \mathbf{S O}(2)$.

Fixando $\delta=\kappa$ como uma anti-simetria, temos $\Gamma_{+}=\mathbf{S O}(2) \equiv \mathbf{S}^{1}$.

1. Vamos encontrar uma base de Hilbert para o anel $\mathcal{P}_{\mathbb{C} \times \mathbb{R}}\left(\Gamma_{+}\right)$. Para isso, considere $p: \mathbb{C} \times \mathbb{R} \rightarrow \mathbb{R}$ um polinômio $\Gamma_{+}$-invariante. Note que podemos escrever $p$ na forma $p(z, x)=\sum a_{\alpha \beta i} z^{\alpha} \bar{z}^{\beta} x^{i}$. Como $\mathbf{S O}(2)$ age em $\mathbb{C} \times \mathbb{R}$ rotacionando a primeira coordenada e fixando a segunda, segue do Exemplo 1.3.4(3), que $p$ é gerado pelas funções

$$
u_{1}(z, x)=z \bar{z} \quad \text { e } \quad u_{2}(z, x)=x .
$$

Portanto $u_{1}$ e $u_{2}$ formam uma base de Hilbert para $\mathcal{P}_{\mathbb{C} \times \mathbb{R}}\left(\Gamma_{+}\right)$ 
2. Precisamos agora encontrar um conjunto gerador para o módulo $\overrightarrow{\mathcal{P}}_{\mathbb{C} \times \mathbb{R}}\left(\Gamma_{+}\right)$sobre o anel $\mathcal{P}_{\mathbb{C} \times \mathbb{R}}\left(\Gamma_{+}\right)$. Seja $q \in \overrightarrow{\mathcal{P}}_{\mathbb{C} \times \mathbb{R}}\left(\Gamma_{+}\right)$. Então podemos escrever $q$ na forma $q(z, x)=\left(q_{1}(z, x), q_{2}(z, x)\right), \operatorname{com} q_{1}: \mathbb{C} \times \mathbb{R} \rightarrow \mathbb{C}$ e $q_{2}: \mathbb{C} \times \mathbb{R} \rightarrow \mathbb{R}$.

Da equivariância de $q$ segue que $q_{2} \in \mathcal{P}_{\mathbb{C} \times \mathbb{R}}\left(\Gamma_{+}\right)$. Ainda, escrevendo $q_{1}(z, x)=$ $\sum a_{\alpha \beta i} z^{\alpha} \bar{z}^{\beta} x^{i}$, também segue da equivariância de $q$ que $q_{1}(z, x)=h_{1}(z, x) z+$ $h_{2}(z, x) z i, \operatorname{com} h_{1}, h_{2} \in \mathcal{P}_{\mathbb{C} \times \mathbb{R}}\left(\Gamma_{+}\right)$. Então,

$$
q(z, x)=h_{1}(z, x)(z, 0)+h_{2}(z, x)(z i, 0)+(0,1) q_{2}(z, x) .
$$

Tome

$$
H_{0}(z, x)=(z, 0), \quad H_{1}(z, x)=(z i, 0) \quad \text { e } \quad H_{2}(z, x)=(0,1) .
$$

Como $H_{0}, H_{1}, H_{2} \in \overrightarrow{\mathcal{P}}_{\mathbb{C} \times \mathbb{R}}\left(\Gamma_{+}\right)$, segue que essas aplicações geram o módulo $\overrightarrow{\mathcal{P}}_{\mathbb{C} \times \mathbb{R}}\left(\Gamma_{+}\right)$ sobre o anel $\mathcal{P}_{\mathbb{C} \times \mathbb{R}}\left(\Gamma_{+}\right)$.

3. Definimos $\tilde{u}_{0}(z, x)=1$ e do Algoritmo 2.3.10 obtemos $\tilde{u}_{1}(z, x)=0$ e $\tilde{u}_{2}(z, x)=x$.

4. Calculamos os geradores para o módulo $\mathcal{P}_{\mathbb{C} \times \mathbb{R}}\left(\Gamma_{+}\right)$sobre o anel $\mathcal{P}_{\mathbb{C} \times \mathbb{R}}(\mathbf{O}(2))$ :

$$
\begin{aligned}
& H_{00}(z, x)=H_{0}(z, x)=(z, 0) \\
& H_{01}(z, x)=H_{1}(z, x)=(z i, 0) \\
& H_{02}(z, x)=H_{2}(z, x)=(0,1) \\
& H_{10}(z, x)=\tilde{u}_{1}(z, x) H_{0}(z, x)=(0,0) \\
& H_{11}(z, x)=\tilde{u}_{1}(z, x) H_{1}(z, x)=(0,0) \\
& H_{12}(z, x)=\tilde{u}_{1}(z, x) H_{2}(z, x)=(0,0) \\
& H_{20}(z, x)=\tilde{u}_{2}(z, x) H_{0}(z, x)=x(z, 0)=(x z, 0) ; \\
& H_{21}(z, x)=\tilde{u}_{2}(z, x) H_{1}(z, x)=x(z i, 0)=(x z i, 0) ; \\
& H_{22}(z, x)=\tilde{u}_{2}(z, x) H_{2}(z, x)=x(0,1)=(0, x) ;
\end{aligned}
$$

5. Agora, do Algoritmo 2.3.13 obtemos os geradores para os reversíveis-equivariantes:

$$
\tilde{H}_{00}(z, x)=(x z, 0), \quad \tilde{H}_{01}(z, x)=(z i, 0), \quad \text { e } \quad \tilde{H}_{02}(z, x)=(0,1) .
$$

\subsection{Forma geral $\mathrm{D}_{n}$-reversível-equivariante}

Considere a ação padrão do grupo diedral $\mathbf{D}_{n} \mathrm{em} \mathbb{C}$, gerada pela conjugação complexa e pela rotação:

$$
\kappa z=\bar{z} \quad \text { e } \quad R_{\frac{2 \pi}{n}} z=e^{i 2 \pi / n} z .
$$

Exemplo 4.5.1. Para $n$ par podemos considerar $\delta=R_{\frac{2 \pi}{n}}$ uma anti-simetria e $\kappa$ uma simetria, de modo que $\Gamma_{+}=\mathbf{D}_{\frac{n}{2}}$. Note que podemos fixar a rotação como anti-simetria, somente quando $n$ é par. De fato, se $n$ é ímpar e a rotação $R_{\frac{2 \pi}{n}}$ é uma anti-simetria, então $\left(R_{\frac{2 \pi}{n}}\right)^{n}$ é uma anti-simetria. Mas isso não pode ocorrer, uma vez que $\left(R_{\frac{2 \pi}{n}}\right)^{n}=I$. 
1. Sabemos pelo Exemplo 1.3.4(4) que $u_{1}(z)=z \bar{z}$ e $u_{2}(z)=z^{\frac{n}{2}}+\bar{z}^{\frac{n}{2}-1}$ formam uma base de Hilbert para o anel $\mathcal{P}\left(\Gamma_{+}\right)$.

2. Também sabemos pelo Exemplo 1.3.17(3) que $H_{0}(z)=z$ e $H_{1}(z)=\bar{z}^{\frac{n}{2}-1}$ geram $\overrightarrow{\mathcal{P}}\left(\Gamma_{+}\right)$como módulo sobre $\mathcal{P}\left(\Gamma_{+}\right)$.

3. Agora, definimos $\tilde{u}_{0}(z)=1$ e do Algoritmo 2.3 .10 obtemos $\tilde{u}_{1}(z)=0$ e $\tilde{u}_{2}(z)=$ $z^{\frac{n}{2}}+\bar{z}^{\frac{n}{2}}$.

4. Calculamos os geradores para o módulo $\overrightarrow{\mathcal{P}}\left(\Gamma_{+}\right)$sobre o anel $\mathcal{P}\left(\mathbf{D}_{n}\right)$ :

$$
\begin{aligned}
& H_{00}(z)=H_{0}(z)=z ; \\
& H_{01}(z)=H_{1}(z)=\bar{z}^{\frac{n}{2}-1} \\
& H_{10}(z)=H_{11}(z)=0 \\
& H_{20}(z)=\tilde{u}_{2}(z) H_{0}(z)=z^{\frac{n}{2}+1}+(z \bar{z}) \bar{z}^{\frac{n}{2}-1} \\
& H_{21}(z)=\tilde{u}_{2}(z) H_{1}(z)=(z \bar{z})^{\frac{n}{2}-1} z+\bar{z}^{n-1} .
\end{aligned}
$$

5. Do Algoritmo 2.3.13 encontramos geradores para o módulo $\overrightarrow{\mathcal{Q}}_{\mathbb{C}}\left(\mathbf{D}_{n}\right)$ sobre o anel $\mathcal{P}_{\mathbb{C}}\left(\mathbf{D}_{n}\right)$ :

$$
\begin{aligned}
\tilde{H}_{01}(z) & =\bar{z}^{\frac{n}{2}-1} \\
\tilde{H}_{20}(z) & =z^{\frac{n}{2}+1}+(z \bar{z}) \bar{z}^{\frac{n}{2}-1} .
\end{aligned}
$$

Observe na Figura 4.3 um exemplo de campo vetorial $\mathbf{D}_{6}$-reversível-equivariante com anti-simetria $R_{\frac{2 \pi}{6}}$, para

$$
\dot{x}=g(x),
$$

onde

$$
g(z)=(z \bar{z}) z^{4}+\bar{z}^{2}=z^{5} \bar{z}+\bar{z}^{2},
$$

o qual, em coordenadas reais se torna

$$
g(x, y)=\left(x^{6}-5 x^{4} y^{2}-5 x^{2} y^{4}+y^{6}+x^{2}-y^{2}, 4 x^{5} y-4 x y^{5}-2 x y\right) .
$$

Outro exemplo é ilustrado na Figura 4.4 para um campo $\mathbf{D}_{4}$-reversível-equivariante, na presença da anti-simetria $R_{\frac{2 \pi}{4}}$, para

$$
\dot{x}=g(x),
$$

onde

$$
g(z)=z^{3}+\bar{z}
$$

o qual, em coordenadas reais se torna

$$
g(x, y)=\left(x+x^{3}-3 x y^{2},-y+3 x^{2} y-y^{3}\right) .
$$

Considere os homomorfismos $\sigma, \tilde{\sigma}: \mathbf{D}_{n} \rightarrow\{-1,1\}$, onde

$$
\sigma\left(R_{\frac{2 \pi}{n}}\right)=\tilde{\sigma}(\kappa)=1
$$




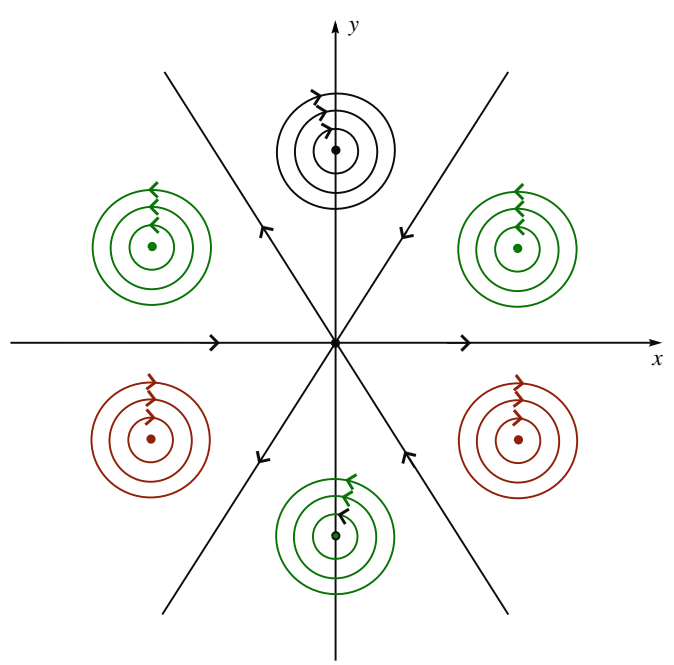

Figura 4.3: Campo vetorial $\mathbf{D}_{6}$-reversível-equivariante dado em 4.3.

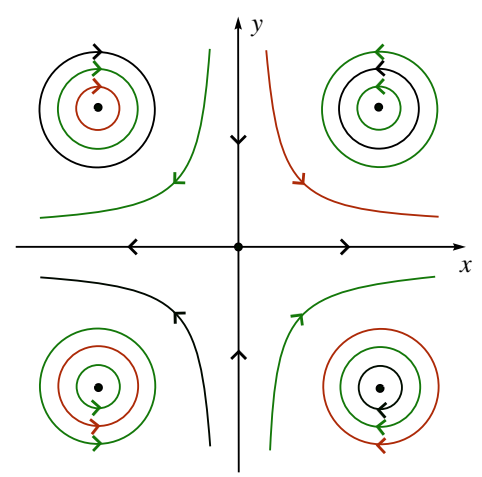

Figura 4.4: Campo vetorial $\mathbf{D}_{4}$-reversível-equivariante dado em 4.4.

Lembramos que uma representação $(V, \rho)$ é $\sigma$-auto-dual se é isomorfa à representação $\left(\rho_{\sigma}, V\right)$. Em outras palavras, se existe um isomorfismo linear $T:(V, \rho) \rightarrow\left(V, \rho_{\sigma}\right)$ tal que $T \in \overrightarrow{\mathcal{Q}}_{V}(\Gamma)$.

Para $\delta=R_{\frac{2 \pi}{n}}$, temos que $\operatorname{tr}(\delta)=2 \cos \frac{2 \pi}{n}$ é nulo se, e somente se, $n=4$. Portanto segue do Lema 2.2.14 que a representação de $\mathbf{D}_{n}$ em $\mathbb{C} \cong \mathbb{R}^{2}$ não é $\tilde{\sigma}$-auto-dual para $n \neq 4$.

Mostramos nos exemplos abaixo, para $n=4$, que a representação $\left(\mathbb{R}^{2}, \rho\right)$ é isomorfa às representações $\left(\mathbb{R}^{2}, \rho_{\sigma}\right)$ e $\left(\mathbb{R}^{2}, \rho_{\tilde{\sigma}}\right)$, respectivamente. De fato, basta tomar os isomorfismos lineares $T_{1}:\left(\mathbb{R}^{2}, \rho\right) \rightarrow\left(\mathbb{R}^{2}, \rho_{\sigma}\right)$ e $T_{2}:\left(\mathbb{R}^{2}, \rho\right) \rightarrow\left(\mathbb{R}^{2}, \rho_{\tilde{\sigma}}\right)$, dados por

$$
T_{1}(x, y)=(y,-x) \quad \text { e } T_{2}(x, y)=(-x, y) .
$$

Logo, a representação $\left(\mathbb{R}^{2}, \rho\right)$ é $\sigma$-auto-dual e $\tilde{\sigma}$-auto-dual. 


\subsubsection{Forma geral $\mathrm{D}_{4}(\kappa)$-reversível-equivariante}

Agora consideramos $\delta=\kappa$ como anti-simetria, obtendo $\Gamma_{+}=\mathbf{Z}_{4}$ :

1. Com auxílio do Singular obtemos a seguinte base de Hilbert para o anel $\mathcal{P}\left(\Gamma_{+}\right)$:

$$
u_{1}(x, y)=x^{2}+y^{2}, \quad u_{2}(x, y)=x^{2} y^{2} \quad \text { e } \quad u_{3}(x, y)=x^{3} y-x y^{3} .
$$

2. Com a técnica do Teorema 1.3 .18 encontramos geradores para $\overrightarrow{\mathcal{P}}\left(\mathbf{D}_{4}\right)$ sobre o anel $\overrightarrow{\mathcal{P}}\left(\mathbf{D}_{4}\right)$ :

$$
\begin{aligned}
& H_{0}(x, y)=(y,-x) \\
& H_{1}(x, y)=(x, y) \\
& H_{2}(x, y)=\left(y^{3},-x^{3}\right) \\
& H_{3}(x, y)=\left(x y^{2}, x^{2} y\right) .
\end{aligned}
$$

3. Definimos $\tilde{u}_{0}(x, y)=1$. Como $u_{1}, u_{2} \in \mathcal{P}\left(\mathbf{D}_{4}\right)=\operatorname{ker} S_{\Gamma_{+}}^{\Gamma}$, temos $\tilde{u}_{1} \equiv \tilde{u}_{2} \equiv 0$, restando apenas $\tilde{u}_{3}(x, y)=x^{3} y-x y^{3}$.

4. Encontramos os geradores para o módulo $\mathcal{P}\left(\Gamma_{+}\right)$sobre o anel $\mathcal{P}\left(\mathbf{D}_{4}\right)$ :

$$
\begin{aligned}
& H_{00}(x, y)=H_{0}(x, y)=(y,-x) \\
& H_{01}(x, y)=H_{1}(x, y)=(x, y) \\
& H_{02}(x, y)=H_{2}(x, y)=\left(y^{3},-x^{3}\right) \\
& H_{03}(x, y)=H_{3}(x, y)=\left(x y^{2}, x^{2} y\right) \\
& H_{30}(x, y)=\tilde{u}_{3}(x, y) H_{0}(x, y)=\left(x^{3} y^{2}-x y^{4},-x^{4} y+x^{2} y^{3}\right) \\
& H_{31}(x, y)=\tilde{u}_{3}(x, y) H_{1}(x, y)=\left(x^{4} y-x^{2} y^{3}, x^{3} y^{2}-x y^{4}\right) \\
& H_{32}(x, y)=\tilde{u}_{3}(x, y) H_{2}(x, y)=\left(x^{3} y^{4}-x y^{6},-x^{6} y+x^{4} y^{3}\right) \\
& H_{33}(x, y)=\tilde{u}_{3}(x, y) H_{3}(x, y)=\left(x^{4} y^{3}-x^{2} y^{5}, x^{5} y^{2}-x^{3} y^{4}\right) .
\end{aligned}
$$

5. Do Algoritmo 2.3.13 obtemos $\tilde{H}_{01} \equiv \tilde{H}_{03} \equiv \tilde{H}_{30} \equiv \tilde{H}_{32} \equiv 0 \mathrm{e}$

$$
\begin{aligned}
& \tilde{H}_{00}(x, y)=(y,-x) \\
& \tilde{H}_{02}(x, y)=H_{02}(x, y)=\left(y^{3},-x^{3}\right) \\
& \tilde{H}_{31}(x, y)=H_{31}(x, y)=\left(x^{4} y-x^{2} y^{3}, x^{3} y^{2}-x y^{4}\right) \\
& \tilde{H}_{33}(x, y)=H_{33}(x, y)=\left(x^{4} y^{3}-x^{2} y^{5}, x^{5} y^{2}-x^{3} y^{4}\right) .
\end{aligned}
$$

Portanto o módulo $\overrightarrow{\mathcal{Q}}\left(\mathbf{D}_{4}\right)$ é gerado sobre o anel $\mathcal{P}\left(\mathbf{D}_{4}\right)$ pelas aplicações $\tilde{H}_{00}, \tilde{H}_{02}, \tilde{H}_{31}$ e $\tilde{H}_{33}$.

\subsubsection{Forma geral $\mathrm{D}_{4}(R)$-reversível-equivariante}

Neste exemplo, fixamos a anti-simetria como sendo a rotação $\delta=R_{\frac{2 \pi}{4}}$, e consideramos a ação de $\mathbf{D}_{4}$ em $\mathbb{R}^{2}$ em vez de $\mathbb{C}$. Dessa forma obtemos $\Gamma_{+}=\mathbf{D}_{2}$. 
1. Com o Singular obtemos uma base de Hilbert para o anel $\mathcal{P}\left(\Gamma_{+}\right)$, dada por $u_{1}(x, y)=x^{2}$ e $u_{2}(x, y)=y^{2}$.

2. Com a técnina do Teorema 1.3 .18 obtemos geradores para o módulo $\overrightarrow{\mathcal{P}}\left(\Gamma_{+}\right)$sobre o anel $\mathcal{P}\left(\Gamma_{+}\right)$, dados por

$$
H_{0}(x, y)=(0, y) \quad \text { e } \quad H_{1}(x, y)=(x, 0) .
$$

3. Definimos $\tilde{u}_{0} \equiv 1$ e pelo Algoritmo 2.3 .10 obtemos $\tilde{u}_{1}(x, y)=-\tilde{u}_{2}(x, y)=x^{2}-y^{2}$.

4. Calculamos os geradores para o módulo $\overrightarrow{\mathcal{P}}\left(\Gamma_{+}\right)$sobre o anel $\mathcal{P}\left(\mathbf{D}_{4}\right)$ :

$$
\begin{aligned}
& H_{00}(x, y)=H_{0}(x, y)=(0, y) \\
& H_{01}(x, y)=H_{1}(x, y)=(x, 0) \\
& H_{10}(x, y)=\tilde{u}_{1}(x, y) H_{0}(x, y)=\left(0, x^{2} y-y^{3}\right) \\
& H_{10}(x, y)=\tilde{u}_{1}(x, y) H_{0}(x, y)=\left(x^{3}-x y^{2}, 0\right)
\end{aligned}
$$

5. Do Algoritmo 2.3.13, obtemos geradores para o módulo $\overrightarrow{\mathcal{Q}}\left(\mathbf{D}_{4}\right)$ sobre o anel $\mathcal{P}\left(\mathbf{D}_{4}\right)$ :

$$
\begin{aligned}
& \tilde{H}_{00}(x, y)=-\tilde{H}_{01}(x, y)=(-x, y) \\
& \tilde{H}_{10}(x, y)=\left(x^{3}-x y^{2}, x^{2} y-y^{3}\right) \\
& \tilde{H}_{11}(x, y)=\left(x^{3}-x y^{3}, y^{3}-x^{3} y\right) .
\end{aligned}
$$

\subsection{Forma geral $\left(\mathbf{D}_{6}+\mathbf{T}^{2}\right) \oplus \mathbf{Z}_{2}$-reversível-equivariante}

Considere $\Gamma=\left(\mathbf{D}_{6} \dot{+} \mathbf{T}^{2}\right) \oplus \mathbf{Z}_{2}$, onde $\dot{+}$ denota a soma semi-direta e $\oplus$ denota uma soma direta. Vamos considerar $\Gamma_{+}=\left(\mathbf{D}_{6}+\mathbf{T}^{2}\right)$. Neste caso, $\Gamma_{-}=\mathbf{Z}_{2}$.

Temos $\mathbf{D}_{6}=\left\langle\mathbf{D}_{3}, \kappa\right\rangle, \mathbf{T}^{2} \cong \mathbf{S}^{1} \times \mathbf{S}^{1}$ e tomamos $\mathbf{Z}_{2}=\langle\delta\rangle$, onde

$$
\delta=\left(\begin{array}{cc}
-1 & 0 \\
0 & -1
\end{array}\right)
$$

Caracterizamos a ação de $\Gamma$ em $\mathbb{C}^{3}$ definindo as ações dos grupos acima em um elemento $\left(z_{1}, z_{2}, z_{3}\right) \in \mathbb{C}^{3}$, da seguinte forma:

$$
\begin{aligned}
\tau\left(z_{1}, z_{2}, z_{3}\right) & =\left(z_{\tau^{-1}(1)}, z_{\tau^{-1}(2)}, z_{\tau^{-1}(3)}\right), \text { para } \tau \in \mathbf{S}_{3} \cong \mathbf{D}_{3} ; \\
\kappa\left(z_{1}, z_{2}, z_{3}\right) & =\left(\bar{z}_{1}, \bar{z}_{2}, \bar{z}_{3}\right) ; \\
\delta\left(z_{1}, z_{2}, z_{3}\right) & =\left(-z_{1},-z_{2},-z_{3}\right) ; \\
(\alpha, \beta)\left(z_{1}, z_{2}, z_{3}\right) & =\left(e^{i \alpha} z_{1}, e^{-i(\alpha+\beta)} z_{2}, e^{i \beta} z_{3}\right), \operatorname{para}(\alpha, \beta) \in \mathbf{S}^{1} \times \mathbf{S}^{1} \cong \mathbf{T}^{2} .
\end{aligned}
$$

Dado $z=\left(z_{1}, z_{2}, z_{3}\right) \in \mathbb{C}^{3}$ seja $v_{j}=z_{j} \bar{z}_{j}$, para $j=1,2,3$. Definimos:

$$
\begin{aligned}
& u_{1}=v_{1}+v_{2}+v_{3} ; \\
& u_{2}=v_{1} v_{2}+v_{1} v_{3}+v_{2} v_{3} ; \\
& u_{3}=v_{1} v_{2} v_{3} ; \\
& u_{4}=z_{1} z_{2} z_{3}+\bar{z}_{1} \bar{z}_{2} \bar{z}_{3} .
\end{aligned}
$$


Observe que $v_{1}, v_{2}, v_{3} \in \mathcal{P}_{\mathbb{C}^{3}}\left(\mathbf{Z}_{2}\right)$ e portanto $u_{1}, u_{2}, u_{3} \in \mathcal{P}_{\mathbb{C}^{3}}\left(\mathbf{Z}_{2}\right)$.

Enunciamos um teorema fundamental para o cálculo dos geradores para o módulo dos $\Gamma$-reversíveis-equivariantes:

Teorema 4.6.1. a) Os elementos $u_{1}, u_{2}, u_{3}$ e $u_{4}$ constituem uma base de Hilbert para o anel $\mathcal{P}_{\mathbb{C}^{3}}\left(\boldsymbol{D}_{6}+\boldsymbol{T}^{2}\right)$.

b) $O$ módulo $\overrightarrow{\mathcal{P}}_{\mathbb{C}^{3}}\left(\boldsymbol{D}_{3}+\boldsymbol{T}^{2}\right)$ é gerado livremente sobre o anel $\mathcal{P}_{\mathbb{C}^{3}}\left(\boldsymbol{D}_{3}+\boldsymbol{T}^{2}\right)$ por:

$$
\begin{gathered}
H_{0}=\left(\begin{array}{l}
z_{1} \\
z_{2} \\
z_{3}
\end{array}\right), H_{1}=\left(\begin{array}{l}
u_{1} z_{1} \\
u_{2} z_{2} \\
u_{3} z_{3}
\end{array}\right), H_{2}=\left(\begin{array}{l}
u_{1}^{2} z_{1} \\
u_{2}^{2} z_{2} \\
u_{3}^{2} z_{3}
\end{array}\right), \\
H_{3}=\left(\begin{array}{l}
\bar{z}_{2} \bar{z}_{3} \\
\bar{z}_{1} \bar{z}_{3} \\
\bar{z}_{1} \bar{z}_{2}
\end{array}\right), H_{4}=\left(\begin{array}{l}
u_{1} \bar{z}_{2} \bar{z}_{3} \\
u_{2} \bar{z}_{1} \bar{z}_{3} \\
u_{3} \bar{z}_{1} \bar{z}_{2}
\end{array}\right), e H_{5}=\left(\begin{array}{l}
u_{1}^{2} \bar{z}_{2} \bar{z}_{3} \\
u_{2}^{2} \bar{z}_{1} \bar{z}_{3} \\
u_{3}^{2} \bar{z}_{1} \bar{z}_{2}
\end{array}\right) .
\end{gathered}
$$

Demonstração: Veja [15], Case Study 4, Theorem 3.1.

Agora, aplicamos o algoritmo para obter os geradores para o módulo das aplicações $\Gamma$-reversíveis-equivariantes.

1. Pelo Teorema 4.6.1, os elementos $u_{1}, u_{2}, u_{3}$ e $u_{4}$ formam uma base de Hilbert para o anel $\mathcal{P}_{\mathbb{C}^{3}}\left(\mathbf{D}_{6}+\mathbf{T}^{2}\right)$.

2. O Teorema 4.6.1 também fornece $H_{0}, H_{1}, H_{2}, H_{3}, H_{4}$ e $H_{5}$ como geradores para o módulo $\overrightarrow{\mathcal{P}}_{\mathbb{C}^{3}}\left(\mathbf{D}_{3}+\mathbf{T}^{2}\right)$ sobre o anel $\mathcal{P}_{\mathbb{C}^{3}}\left(\mathbf{D}_{3}+\mathbf{T}^{2}\right)$.

3. Definimos $\tilde{u}_{0} \equiv 1$. Do Algoritmo 2.3 .10 obtemos $\tilde{u}_{i} \equiv 0$ para $i=1,2,3$ e $\tilde{u}_{4}=u_{4}$.

4. Calculamos os geradores para o módulo $\overrightarrow{\mathcal{P}}_{\mathbb{C}^{3}}\left(\mathbf{D}_{6} \dot{+} \mathbf{T}^{2}\right)$ sobre o anel $\mathcal{P}_{\mathbb{C}^{3}}\left(\left(\mathbf{D}_{6} \dot{+}\right.\right.$ $\left.\left.\mathbf{T}^{2}\right) \oplus \mathbf{Z}_{2}\right)$ :

$$
\begin{aligned}
& H_{00}(z)=H_{0}(z) ; \\
& H_{01}(z)=H_{1}(z) ; \\
& H_{02}(z)=H_{2}(z) ; \\
& H_{03}(z)=H_{3}(z) ; \\
& H_{04}(z)=H_{4}(z) ; \\
& H_{05}(z)=H_{5}(z) ; \\
& H_{40}(z)=u_{4}(z) H_{0}(z) ; \\
& H_{41}(z)=u_{4}(z) H_{1}(z) ; \\
& H_{42}(z)=u_{4}(z) H_{2}(z) ; \\
& H_{43}(z)=u_{4}(z) H_{3}(z) ; \\
& H_{44}(z)=u_{4}(z) H_{4}(z) ; \\
& H_{45}(z)=u_{4}(z) H_{5}(z) .
\end{aligned}
$$


5. Por fim, pelo Algoritmo 2.3.13 obtemos $\tilde{H}_{00} \equiv \tilde{H}_{01} \equiv \tilde{H}_{02} \equiv \tilde{H}_{40} \equiv \tilde{H}_{41} \equiv \tilde{H}_{42} \equiv$ 0 e

$$
\begin{aligned}
\tilde{H}_{03}\left(z_{1}, z_{2}, z_{3}\right) & =\left(\bar{z}_{2} \bar{z}_{3}, \bar{z}_{1} \bar{z}_{3}, \bar{z}_{1} \bar{z}_{2}\right) ; \\
\tilde{H}_{04}\left(z_{1}, z_{2}, z_{3}\right) & =\left(u_{1} \bar{z}_{2} \bar{z}_{3}, u_{2} \bar{z}_{1} \bar{z}_{3}, u_{3} \bar{z}_{1} \bar{z}_{2}\right) ; \\
\tilde{H}_{05}\left(z_{1}, z_{2}, z_{3}\right) & =\left(u_{1}^{2} \bar{z}_{2} \bar{z}_{3}, u_{2}^{2} \bar{z}_{1} \bar{z}_{3}, u_{3}^{2} \bar{z}_{1} \bar{z}_{2}\right) ; \\
\tilde{H}_{40}\left(z_{1}, z_{2}, z_{3}\right) & =\left(u_{4}, z_{1}, u_{4} z_{2}, u_{4} z_{3}\right) ; \\
\tilde{H}_{41}\left(z_{1}, z_{2}, z_{3}\right) & =\left(u_{4} u_{1} z_{1}, u_{4} u_{2} z_{2}, u_{4} u_{3} z_{3}\right) ; \\
\tilde{H}_{42}\left(z_{1}, z_{2}, z_{3}\right) & =\left(u_{4} u_{1}^{2} z_{1}, u_{4} u_{2}^{2} z_{2}, u_{4} u_{3}^{2} z_{3}\right) .
\end{aligned}
$$

\subsection{Forma geral $\mathbb{O}$-reversível-equivariante}

Seja $V=\mathbb{R}^{3}$, e considere $\Gamma=\mathbb{O}$, o grupo octaedral de simetrias do cubo agindo em $\mathbb{R}^{3}$ pela ação padrão, dada pela multiplicação de matrizes . O grupo octaedral possui 48 elementos, sendo 24 simetrias rotacionais e 24 reflexões, e seus geradores são:

$$
\kappa_{x}=\left(\begin{array}{ccc}
-1 & 0 & 0 \\
0 & 1 & 0 \\
0 & 0 & 1
\end{array}\right), \quad R_{x}=\left(\begin{array}{ccc}
1 & 0 & 0 \\
0 & 0 & -1 \\
0 & 1 & 0
\end{array}\right), \quad R_{y}=\left(\begin{array}{ccc}
0 & 0 & 1 \\
0 & 1 & 0 \\
-1 & 0 & 0
\end{array}\right) .
$$

Esses elementos representam a reflexão em relação ao plano $x=0$ e rotações de ângulo $\frac{\pi}{2}$ sobre os eixos $x$ e $y$ respectivamente. Consideramos $\delta=\kappa_{x}$ uma anti-simetria, $R_{x}$ e $R_{y}$ como simetrias. Dessa forma temos $\Gamma=\Gamma_{+} \dot{U} \Gamma_{-}, \operatorname{com} \Gamma_{-}=\delta \Gamma_{+}$e $\Gamma_{+}=$ $\left\langle R_{x}, R_{y}\right\rangle \cong \mathbf{S}_{4}$, o qual representa o grupo de simetrias do Tetraedro, composto por 12 simetrias rotacionais e 12 reflexões. Aplicamos o algoritmo:

1. Com o auxílio do Singular obtemos uma base de Hilbert $\left\{u_{1}, u_{2}, u_{3}, u_{4}\right\}$ para o anel $\mathcal{P}\left(\Gamma_{+}\right)$, onde:

$$
\begin{aligned}
& u_{1}\left(x_{1}, x_{2}, x_{3}\right)=x_{1}^{2}+x_{2}^{2}+x_{3}^{2} \\
& u_{2}\left(x_{1}, x_{2}, x_{3}\right)=x_{1}^{2} x_{2}^{2}+x_{1}^{2} x_{3}^{2}+x_{2}^{2} x_{3}^{2} \\
& u_{3}\left(x_{1}, x_{2}, x_{3}\right)=x_{1}^{2} x_{2}^{2} x_{3}^{2} \\
& u_{4}\left(x_{1}, x_{2}, x_{3}\right)=x_{1}^{5}\left(x_{2}^{3} x_{3}-x_{2} x_{3}^{3}\right)+x_{2}^{5}\left(x_{1} x_{3}^{3}-x_{1}^{3} x_{3}\right)+x_{3}^{5}\left(x_{1}^{3} x_{2}-x_{1} x_{2}^{3}\right) .
\end{aligned}
$$

2. Utilizando a técnica do Teorema 1.3 .18 encontramos geradores para o módulo $\overrightarrow{\mathcal{P}}\left(\Gamma_{+}\right)$sobre o anel $\mathcal{P}\left(\Gamma_{+}\right)$, os quais são dados por:

$$
\begin{aligned}
& H_{0}=\left(x_{1}, x_{2}, x_{3}\right) \\
& H_{1}=\left(x_{1}\left(x_{2}^{2}+x_{3}^{2}\right), x_{2}\left(x_{1}^{2}+x_{3}^{2}\right), x_{3}\left(x_{1}^{2}+x_{2}^{2}\right)\right) \\
& H_{2}=\left(x_{2}^{3} x_{3}-x_{2} x_{3}^{3}, x_{1} x_{3}^{3}-x_{1}^{3} x_{3}, x_{1}^{3} x_{2}-x_{1} x_{2}^{3}\right) \\
& H_{3}=\left(x_{1}\left(x_{2}^{4}+x_{3}^{4}\right), x_{2}\left(x_{1}^{4}+x_{3}^{4}\right), x_{3}\left(x_{1}^{4}+x_{2}^{4}\right)\right) \\
& H_{4}=\left(x_{1}^{2}\left(x_{2}^{3} x_{3}-x_{2} x_{3}^{3}\right), x_{2}^{2}\left(x_{1} x_{3}^{3}-x_{1}^{3} x_{3}\right), x_{3}^{2}\left(x_{1}^{3} x_{2}-x_{1} x_{2}^{3}\right)\right) \\
& H_{5}=\left(x_{1}^{4}\left(x_{2}^{3} x_{3}-x_{2} x_{3}^{3}\right), x_{2}^{4}\left(x_{1} x_{3}^{3}-x_{1}^{3} x_{3}\right), x_{3}^{4}\left(x_{1}^{3} x_{2}-x_{1} x_{2}^{3}\right)\right) .
\end{aligned}
$$


3. Definimos $\tilde{u}_{0} \equiv 1$ e do algoritmo 2.3 .10 obtemos: $\tilde{u}_{2} \equiv \tilde{u}_{3} \equiv 0$ e

$$
\tilde{u}_{4}\left(x_{1}, x_{2}, x_{3}\right)=x_{1}^{5}\left(x_{2}^{3} x_{3}-x_{2} x_{3}^{3}\right)+x_{2}^{5}\left(x_{1} x_{3}^{3}-x_{1}^{3} x_{3}\right)+x_{3}^{5}\left(x_{1}^{3} x_{2}-x_{1} x_{2}^{3}\right) .
$$

4. Segue o conjunto $\left\{H_{0}, H_{1}, H_{2}, H_{3}, H_{4}, H_{5}, \tilde{u}_{4} H_{0}, \tilde{u}_{4} H_{1}, \tilde{u}_{4} H_{2}, \tilde{u}_{4} H_{3}, \tilde{u}_{4} H_{4}, \tilde{u}_{4} H_{5}\right\}$ gera o módulo $\overrightarrow{\mathcal{P}}\left(\Gamma_{+}\right)$sobre o anel $\mathcal{P}(\Gamma)$.

5. Pelo Algoritmo 2.3.13 obtemos geradores para o módulo $\overrightarrow{\mathcal{Q}}(\mathbb{O})$ sobre o anel $\overrightarrow{\mathcal{P}}(\mathbb{O})$ :

$$
\begin{aligned}
\tilde{H}_{02}= & \left(x_{2}^{3} x_{3}-x_{2} x_{3}^{3}, x_{1} x_{3}^{3}-x_{1}^{3} x_{3}, x_{1}^{3} x_{2}-x_{1} x_{2}^{3}\right) ; \\
\tilde{H}_{04}= & \left(x_{1}^{2}\left(x_{2}^{3} x_{3}-x_{2} x_{3}^{3}\right), x_{2}^{2}\left(x_{1} x_{3}^{3}-x_{1}^{3} x_{3}\right), x_{3}^{2}\left(x_{1}^{3} x_{2}-x_{1} x_{2}^{3}\right)\right) ; \\
\tilde{H}_{05}= & \left(x_{1}^{4}\left(x_{2}^{3} x_{3}-x_{2} x_{3}^{3}\right), x_{2}^{4}\left(x_{1} x_{3}^{3}-x_{1}^{3} x_{3}\right), x_{3}^{4}\left(x_{1}^{3} x_{2}-x_{1} x_{2}^{3}\right)\right) ; \\
\tilde{H}_{40}= & \left(x_{1}^{6}\left(x_{2}^{3} x_{3}-x_{2} x_{3}^{3}\right)+x_{2}^{5}\left(x_{1}^{2} x_{3}^{3}-x_{1}^{4} x_{3}\right)+x_{3}^{5}\left(x_{1}^{4} x_{2}-x_{2} x_{2}^{3}\right),\right. \\
& x_{1}^{5}\left(x_{2}^{4} x_{3}-x_{2}^{2} x_{3}^{3}\right)+x_{2}^{6}\left(x_{1} x_{3}^{3}-x_{1}^{3} x_{3}\right)+x_{3}^{5}\left(x_{1}^{3} x_{2}^{2}-x_{1} x_{2}^{4}\right), \\
& \left.x_{1}^{5}\left(x_{2}^{3} x_{3}^{2}-x_{2} x_{3}^{4}\right)+x_{2}^{5}\left(x_{1} x_{3}^{4}-x_{1}^{3} x_{3}^{2}\right)+x_{3}^{6}\left(x_{1}^{3} x_{2}-x_{1} x_{2}^{3}\right)\right) ; \\
\tilde{H}_{41}= & \left(x_{2}^{2}+x_{3}^{2}\right)\left(x_{1}^{6}\left(x_{2}^{3} x_{3}-x_{2} x_{3}^{3}\right)+x_{2}^{5}\left(x_{1}^{2} x_{3}^{3}-x_{1}^{4} x_{3}\right)+x_{3}^{5}\left(x_{1}^{4} x_{2}-x_{2} x_{2}^{3}\right)\right), \\
& \left(x_{1}^{2}+x_{3}^{2}\right)\left(x_{1}^{5}\left(x_{2}^{4} x_{3}-x_{2}^{2} x_{3}^{3}\right)+x_{2}^{6}\left(x_{1} x_{3}^{3}-x_{1}^{3} x_{3}\right)+x_{3}^{5}\left(x_{1}^{3} x_{2}^{2}-x_{1} x_{2}^{4}\right)\right), \\
& \left.\left(x_{1}^{2}+x_{2}^{2}\right)\left(x_{1}^{5}\left(x_{2}^{3} x_{3}^{2}-x_{2} x_{3}^{4}\right)+x_{2}^{5}\left(x_{1} x_{3}^{4}-x_{1}^{3} x_{3}^{2}\right)+x_{3}^{6}\left(x_{1}^{3} x_{2}-x_{1} x_{2}^{3}\right)\right)\right) ; \\
\tilde{H}_{43}= & \left(\left(x_{2}^{4}+x_{3}^{4}\right)\left(x_{1}^{6}\left(x_{2}^{3} x_{3}-x_{2} x_{3}^{3}\right)+x_{2}^{5}\left(x_{1}^{2} x_{3}^{3}-x_{1}^{4} x_{3}\right)+x_{3}^{5}\left(x_{1}^{4} x_{2}-x_{2} x_{2}^{3}\right)\right),\right. \\
& \left(x_{1}^{4}+x_{3}^{4}\right)\left(x_{1}^{5}\left(x_{2}^{4} x_{3} x_{2}^{2} x_{3}^{3}\right)+x_{2}^{6}\left(x_{1} x_{3}^{3}-x_{1}^{3} x_{3}\right)+x_{3}^{5}\left(x_{1}^{3} x_{2}^{2}-x_{1} x_{2}^{4}\right)\right), \\
& \left.\left(x_{1}^{4}+x_{2}^{4}\right)\left(x_{1}^{5}\left(x_{2}^{3} x_{3}^{2}-x_{2} x_{3}^{4}\right)+x_{2}^{5}\left(x_{1} x_{3}^{4}-x_{1}^{3} x_{3}^{2}\right)+x_{3}^{6}\left(x_{1}^{3} x_{2}-x_{1} x_{2}^{3}\right)\right)\right),
\end{aligned}
$$

\subsection{Forma geral $\mathrm{S}_{n}$-reversível-equivariante}

Considere a ação do grupo das permutações de $n$ elementos $\Gamma=\mathbf{S}_{n}$, em $\mathbb{R}^{n}$ definida por:

$$
\tau x=\left(x_{\tau^{-1}(1)}, \ldots, x_{\tau^{-1}(n)}\right),
$$

para $\tau \in \mathbf{S}_{n}$ e $x=\left(x_{1}, \ldots, x_{n}\right) \in \mathbb{R}^{n}$.

Definição 4.8.1. Uma permutação $\tau \in S_{n}$ é chamada de $r$-ciclo se existem elementos distinhos $a_{1}, \ldots, a_{r} \in\{1, \ldots, n\}$ tais que $\tau\left(a_{1}\right)=a_{2}, \ldots, \tau\left(a_{r-1}\right)=a_{r}, \tau\left(a_{r}\right)=a_{1} e$ tais que $\tau(j)=j$ para todo $j \in\{1, \ldots, n\}\left\{a_{1}, \ldots, a_{r}\right\}$. Denotamos tal elemento por $\left(a_{1} a_{2} \ldots a_{r}\right)$.

Observe que se $\tau \in \mathbf{S}_{n}$ é um $r$-ciclo, então $\tau^{r}=i d$. Um elemento $\tau=(i j) \in \mathbf{S}_{n}$ também é chamado de transposição.

Definição 4.8.2. Uma permutação $\tau \in \boldsymbol{S}_{n}$ é par (ímpar) se pode ser escrita como um produto de um número par(ímpar) de transposiçôes.

Em $\mathbf{S}_{n}$ defimos o conjunto $A_{n}$ formado por todas as permutações pares, e definimos um homomorfismo sobrejetor $\sigma: \mathbf{S}_{n} \rightarrow\{-1,1\}$ dado por: $\sigma(\tau)=1$ se $\tau$ é par e $\sigma(\tau)=-1$ se $\tau$ é ímpar. 
Observe que $\Gamma_{+}=\operatorname{ker} \sigma=A_{n}$ é um subgrupo normal de $\mathbf{S}_{n}$ de índice 2, chamado de subgrupo alternado.

Em [13](página 210), Garcia mostra que para todo $n \geqslant 3$, o grupo alternado $A_{n}$ é o único subgrupo de $\mathbf{S}_{n}$ de índice 2. Segue daí que o homomorfismo $\sigma$ definido acima é único, e que o único subgrupo de simetrias em $\mathbf{S}_{n}$ é o grupo alternado $A_{n}$, para todo $n \geqslant 3$. Ainda, é apresentado a forma dos geradores do grupo $A_{n}$ na seguinte proposição:

Proposição 4.8.3. Sejam $a, b \in\{1,2, \ldots, n ;\}, a \neq b$. Então para todo $n \geq 3$,

$$
A_{n}=\langle\{(a b l) \text { para } l=1,2, \ldots, n ; l \neq a, b\}\rangle .
$$

Demonstração: Veja Garcia [13], Capítulo V, Proposição V.10.19.

Outro resultado importante é provado por Amstrong:

Teorema 4.8.4. O grupo $\boldsymbol{S}_{n}$ é gerado pelas permutações (12) e (12 ...n);

Demonstração: Veja Armstrong [1], Theorem 6.3.

Para aplicar os Algoritmos 2.3.10 e 2.3.13 fixamos $\delta=(12)$ como anti-simetria, e ilustramos essas idéias nas próximas subseções com exemplos para $n=2$ e $n=3$. Os demais casos não foram abordados aqui, pois o programa Singular apresenta falha crítica para $n \geq 4$.

\subsubsection{Forma geral $\mathrm{S}_{2}$-reversível-equivariante}

Para $n=2$, temos $\mathbf{S}_{2}=\{(12), I\}$ agindo em $\mathbb{R}^{2}$ e, portanto, a anti-simetria é $\delta=(12)$ e $\Gamma_{+}=\{I\}$. Portanto, no plano $\delta$ representa a reflexão:

$$
\delta=\left(\begin{array}{ll}
0 & 1 \\
1 & 0
\end{array}\right) .
$$

Vamos aplicar o algoritmo para o grupo $\mathbf{S}_{2}$ :

1. Como $\Gamma_{+}=\{I\}$, então uma base de Hilbert $\left\{u_{1}, u_{2}\right\}$ para este anel é dada por $u_{1}(x, y)=x$ e $u_{2}(x, y)=y$.

2. Se $p \in \overrightarrow{\mathcal{P}}\left(\Gamma_{+}\right)$, então $p(x, y)=\left(p_{1}(x, y), p_{2}(x, y)\right)=p_{1}(x, y)(1,0)+p_{2}(x, y)(0,1)$. Note que $p_{1}, p_{2} \in \mathcal{P}\left(\Gamma_{+}\right)$, e como $H_{0} \equiv(1,0), H_{1} \equiv(0,1)$ são $\Gamma_{+}$-equivariantes, segue que $\overrightarrow{\mathcal{P}}\left(\Gamma_{+}\right)$é gerado como módulo sobre o anel $\mathcal{P}\left(\Gamma_{+}\right)$pelas aplicações $H_{0} \mathrm{e}$ $H_{1}$.

3. Definimos $\tilde{u}_{0} \equiv 1$ e pelo Algoritmo 2.3.10 encontamos $\tilde{u}_{1}(x, y)=-\tilde{u}_{2}(x, y)=$ $\frac{1}{2}(x-y)$. Sem perda de generalidade podemos considerar $\tilde{u}_{1}(x, y)=(x-y)$.

4. Agora, calculamos os geradores do módulo $\overrightarrow{\mathcal{P}}\left(\Gamma_{+}\right)$sobre o anel $\mathcal{P}(\Gamma)$ :

$$
\begin{aligned}
& H_{00}(x, y)=H_{0}(x, y)=(1,0) ; \\
& H_{01}(x, y)=H_{1}(x, y)=(0,1) ; \\
& H_{10}(x, y)=\tilde{u}_{1}(x, y) H_{0}(x, y)=(x-y, 0) ; \\
& H_{11}(x, y)=\tilde{u}_{1}(x, y) H_{1}(x, y)=(0, x-y) .
\end{aligned}
$$


5. Finalmente, do Algoritmo 2.3.13 obtemos geradores para o módulo dos $\mathbf{S}_{2}$-reversíveisequivariantes:

$$
\begin{aligned}
\tilde{H}_{00}(x, y) & =-\tilde{H}_{01}(x, y)=(1,-1) \\
\tilde{H}_{10}(x, y) & =\tilde{H}_{11}(x, y)=(x-y, y-x) .
\end{aligned}
$$

\subsubsection{Forma geral $\mathrm{S}_{3}$-reversível-equivariante}

Seja $\mathbf{S}_{3}=\langle(12),(123)\rangle$, agindo em $\mathbb{R}^{3}$. Fixando $\delta=(12)$ como uma anti-simetria, temos $\Gamma_{+}=A_{3}=\langle(123)\rangle$. Observe que o gerador do grupo altermado $A_{3}$ corresponde à matriz

$$
\left(\begin{array}{lll}
0 & 1 & 0 \\
1 & 0 & 0 \\
0 & 0 & 1
\end{array}\right)
$$

Vamos aplicar a algoritmo para o cálculo dos geradores para o módulo $\overrightarrow{\mathcal{Q}}\left(\mathbf{S}_{3}\right)$.

1. Com ajuda do programa singular obtemos uma base de Hilbert para o anel $\mathcal{P}\left(A_{3}\right)$, dada por

$$
\begin{aligned}
& u_{1}\left(x_{1}, x_{2}, x_{3}\right)=x_{1}+x_{2}+x_{3} ; \\
& u_{2}\left(x_{1}, x_{2}, x_{3}\right)=x_{1} x_{2}+x_{1} x_{3}+x_{2} x_{3} ; \\
& u_{3}\left(x_{1}, x_{2}, x_{3}\right)=x_{1} x_{2} x_{3} ; \\
& u_{4}\left(x_{1}, x_{2}, x_{3}\right)=x_{1} x_{2}^{2}+x_{1}^{2} x_{3}+x_{2} x_{3}^{2} .
\end{aligned}
$$

2. Recorremos à técnica do Teorema 1.3.18, para encontrar geradores para o anel $\overrightarrow{\mathcal{P}}\left(A_{3}\right)$ sobre o anel $\mathcal{P}\left(A_{3}\right)$ :

$$
\begin{aligned}
& H_{0}\left(x_{1}, x_{2}, x_{3}\right)=\left(x_{3}, x_{1}, x_{2}\right) ; \\
& H_{1}\left(x_{1}, x_{2}, x_{3}\right)=\left(x_{2}, x_{3}, x_{1}\right) ; \\
& H_{2}\left(x_{1}, x_{2}, x_{3}\right)=\left(x_{3}^{2}, x_{1}^{2}, x_{2}^{2}\right) ; \\
& H_{3}\left(x_{1}, x_{2}, x_{3}\right)=\left(x_{1} x_{3}, x_{1} x_{2}, x_{2} x_{3}\right) ;
\end{aligned}
$$

3. Definimos $\tilde{u}_{0} \equiv 1$. do Algoritmo 2.3 .10 obtemos $\tilde{u}_{1} \equiv \tilde{u}_{2} \equiv \tilde{u}_{3} \equiv 0$ e

$$
\tilde{u}_{4}\left(x_{1}, x_{2}, x_{3}\right)=\frac{1}{2}\left(x_{1}^{2}\left(x_{3}-x_{2}\right)+x_{2}^{2}\left(x_{1}-x_{3}\right)+x_{3}^{2}\left(x_{2}-x_{1}\right)\right) .
$$

Sem perda de generalidade podemos assumir

$$
\tilde{u}_{4}\left(x_{1}, x_{2}, x_{3}\right)=x_{1}^{2}\left(x_{3}-x_{2}\right)+x_{2}^{2}\left(x_{1}-x_{3}\right)+x_{3}^{2}\left(x_{2}-x_{1}\right) .
$$

4. Calculamos geradores $\tilde{u}_{i} H_{j}$, para $i=0,4$ e $j=0,1,2,3$, para o módulo $\overrightarrow{\mathcal{P}}\left(A_{3}\right)$ 
sobre o anel $\mathcal{P}\left(\mathbf{S}_{3}\right)$ :

$$
\begin{aligned}
& H_{00}\left(x_{1}, x_{2}, x_{3}\right)=H_{0}\left(x_{1}, x_{2}, x_{3}\right)=\left(x_{3}, x_{1}, x_{2}\right) ; \\
& H_{01}\left(x_{1}, x_{2}, x_{3}\right)=H_{1}\left(x_{1}, x_{2}, x_{3}\right)=\left(x_{2}, x_{3}, x_{1}\right) ; \\
& H_{02}\left(x_{1}, x_{2}, x_{3}\right)=H_{2}\left(x_{1}, x_{2}, x_{3}\right)=\left(x_{3}^{2}, x_{1}^{2}, x_{2}^{2}\right) ; \\
& H_{03}\left(x_{1}, x_{2}, x_{3}\right)=H_{3}\left(x_{1}, x_{2}, x_{3}\right)=\left(x_{1} x_{3}, x_{1} x_{2}, x_{2} x_{3}\right) ; \\
& H_{40}\left(x_{1}, x_{2}, x_{3}\right)=\left(x_{1}^{2}\left(x_{3}-x_{2}\right)+x_{2}^{2}\left(x_{1}-x_{3}\right)+x_{3}^{2}\left(x_{2}-x_{1}\right)\right)\left(x_{3}, x_{1}, x_{2}\right) ; \\
& H_{41}\left(x_{1}, x_{2}, x_{3}\right)=\left(x_{1}^{2}\left(x_{3}-x_{2}\right)+x_{2}^{2}\left(x_{1}-x_{3}\right)+x_{3}^{2}\left(x_{2}-x_{1}\right)\right)\left(x_{2}, x_{3}, x_{1}\right) ; \\
& H_{42}\left(x_{1}, x_{2}, x_{3}\right)=\left(x_{1}^{2}\left(x_{3}-x_{2}\right)+x_{2}^{2}\left(x_{1}-x_{3}\right)+x_{3}^{2}\left(x_{2}-x_{1}\right)\right)\left(x_{3}^{2}, x_{1}^{2}, x_{2}^{2}\right) ; \\
& H_{43}\left(x_{1}, x_{2}, x_{3}\right)=\left(x_{1}^{2}\left(x_{3}-x_{2}\right)+x_{2}^{2}\left(x_{1}-x_{3}\right)+x_{3}^{2}\left(x_{2}-x_{1}\right)\right)\left(x_{1} x_{3}, x_{1} x_{2}, x_{2} x_{3}\right) .
\end{aligned}
$$

5. Pelo Algoritmo 2.3.13, encontramos geradores para o módulo $\overrightarrow{\mathcal{Q}}\left(\mathbf{S}_{3}\right)$ sobre o anel $\mathcal{P}\left(\mathbf{S}_{3}\right)$ :

$$
\begin{aligned}
& \tilde{H}_{01}\left(x_{1}, x_{2}, x_{3}\right)=\left(x_{2}-x_{3}, x_{3}-x_{1}, x_{1}-x_{2}\right)=-\tilde{H}_{00}\left(x_{1}, x_{2}, x_{3}\right) ; \\
& \tilde{H}_{02}\left(x_{1}, x_{2}, x_{3}\right)=\left(x_{3}^{2}-x_{2}^{2}, x_{1}^{2}-x_{3}^{2}, x_{2}^{2}-x_{1}^{2}\right) \text {; } \\
& \tilde{H}_{03}\left(x_{1}, x_{2}, x_{3}\right)=\left(x_{1}\left(x_{3}-x_{2}\right), x_{2}\left(x_{1}-x_{3}\right), x_{3}\left(x_{2}-x_{1}\right)\right) \text {; } \\
& \tilde{H}_{40}\left(x_{1}, x_{2}, x_{3}\right)=\left(x_{1}^{2}\left(x_{1} x_{3}+x_{2}\right)\left(x_{3}-x_{2}\right)+x_{2}^{2}\left(x_{3}+x_{2}\right)\left(x_{1}-x_{3}\right)+x_{3}^{2}\left(x_{3}+x_{2}\right)\left(x_{1}-x_{2}\right)\right. \text {, } \\
& x_{1}^{2}\left(x_{1}+x_{3}\right)\left(x_{3}-x_{2}\right)+x_{2}^{2}\left(x_{1}+x_{2} x_{3}\right)\left(x_{1}-x_{3}\right)+x_{3}^{2}\left(x_{1}+x_{3}\right)\left(x_{2}-x_{1}\right), \\
& \left.x_{1}^{2}\left(x_{2}+x_{1}\right)\left(x_{3}-x_{2}\right)+x_{2}^{2}\left(x_{2}+x_{1}\right)\left(x_{1}-x_{3}\right)+x_{3}^{2}\left(x_{1}+x_{3}\right)\left(x_{2}^{2}-x_{1}^{2}\right)\right) ; \\
& \tilde{H}_{41}\left(x_{1}, x_{2}, x_{3}\right)=\left(x_{1}^{2}\left(x_{3}^{2}-x_{2}^{2}\right)+x_{2}^{2}\left(x_{2}+x_{3}\right)\left(x_{1}-x_{3}\right)+x_{3}^{2}\left(x_{2}+1\right)\left(x_{2}-x_{1}\right)\right. \text {, } \\
& x_{1}^{2}\left(x_{3}+1\right)\left(x_{3}-x_{2}\right)+x_{2}^{2}\left(x_{1}^{2}-x_{3}^{2}\right)+x_{3}^{2}\left(1+x_{1}\right)\left(x_{2}-x_{1}\right), \\
& \left.x_{1}^{2}\left(x_{1}+x_{2}\right)\left(x_{3}-x_{2}\right)+x_{2}^{2}\left(x_{1}-x_{2}\right)\left(x_{1}-x_{3}\right)+x_{3}^{2}\left(x_{2}^{2}-x_{1}\right)\right) \text {; } \\
& \tilde{H}_{42}\left(x_{1}, x_{2}, x_{3}\right)=\left(x_{1}^{2}\left(x_{2}-x_{2}\right)\left(x_{3}-x_{2}\right)+x_{2}^{2}\left(x_{3}^{2}-x_{2}^{2}\right)\left(x_{1}-x_{3}\right)+x_{3}^{2}\left(x_{3}^{2}-x_{2}^{2}\right)\left(x_{2}-x_{1}\right)\right. \text {, } \\
& \left(x_{1}^{2}\left(x_{1}^{2}-x_{3}^{2}\right)\left(x_{3}-x_{2}\right)+x_{2}^{(} x_{1}^{2}-x_{3}\right)\left(x_{1}-x_{3}\right)+x_{3}^{2}\left(x_{1}^{2}-x_{3}^{2}\right)\left(x_{2}-x_{1}\right) \text {, } \\
& \left.x_{1}^{2}\left(x_{2}^{2}-x_{1}^{2}\right)\left(x_{3}-x_{2}\right)+x_{2}^{2} x_{3}\left(x_{2}-x_{1}\right)\left(x_{1}-x_{3}\right)+x_{3}^{3}\left(x_{2}-x_{1}\right)\left(x_{2}-x_{1}\right)\right) ; \\
& \tilde{H}_{43}\left(x_{1}, x_{2}, x_{3}\right)=\left(x_{1}^{3}\left(x_{3}-x_{2}\right)^{2}+x_{1} x_{2}^{2}\left(x_{3}-x_{1} x_{2}\right)\left(x_{1}-x_{3}\right)+x_{1} x_{3}^{2}\left(x_{3}-x_{2}\right)\left(x_{2}-x_{1}\right)\right. \text {, } \\
& x_{1}^{2} x_{2}\left(x_{1}-x_{3}\right)\left(x_{3}-x_{2}\right)+x_{2}^{3}\left(x_{1}-x_{3}\right)^{2}+x_{2} x_{3}^{2}\left(x_{1}-x_{2} x_{3}\right)\left(x_{2}-x_{1}\right), \\
& \left.x_{1}^{2} x_{3}\left(x_{2}-x_{1}\right)\left(x_{2}-x_{3}\right)+x_{2}^{2} x_{3}\left(x_{2}-x_{1}\right)\left(x_{1}-x_{3}\right)+x_{3}^{3}\left(x_{2}-x_{1}\right)^{2}\right) \text {. }
\end{aligned}
$$




\section{Bibliografia}

[1] Armstrong, M. A. [1988] Groups and Symmetry. Undergraduate Texts in Mathematics, Springer-Verlag, New York.

[2] Antoneli, F., Dias, A. P. S. e Matthews, P. C. [2007] Invariants, Equivariants and Characters in Symmetric Bifurcation Theory. Proceedings of the Royal Society of Edinburgh. Section A, Mathematics, 137, 01-36.

[3] Antoneli, F., Baptistelli, P. H., Dias, A. P. S. e Manoel, M. G. [2009] Invariant theory and reversible-equivariant vector fields. Journal of Pure and Applied Algebra (A Aparecer, disponível online em http://www.sciencedirect.com).

[4] Baptistelli, P. H. [2007] Singularidades e teoria de invariantes em bifurcação reversivel-equivariante. Tese de doutorado, ICMC-USP.

[5] Baptistelli, P. H. e Manoel, M. G. [2005] Some results on reversible-equivariant vector fields. Cadernos de Matemática 6, 237-263.

[6] Bröcker, T. e Dieck, T. tom [1995] Representations of compact Lie groups. Graduate Texts in Mathematics 98, Spreing-Verlag, Berlin-Heidelberg, New York.

[7] Buono, P. L., Lamb, J. S. W. e Roberts, R. M. [2007] Bifurcation and branching of equilibria of reversible equivariant vector fields. Em preparação.

[8] Buzzi, C. A. e Lamb, J. S. W. [2005] Reversible equivariant Hopf bifurcation. Archive for Rational Mechanics and Analysis, 175, 39-84.

[9] Dias, A. P. S. [1997] Bifurcations with wreath product simmetry. Phd thesis, University of Warwick, Inglaterra.

[10] Dionne, B., Golubitsky, M. e Stewart, I. [1996] Coupled cells with internal symmetry Part I: wreath products Nonlinearity. 9 559-574.

[11] Doering, C. I. e Lopes, A. O. [2005] Equações diferenciais ordinárias. Coleção matemática universitária, Rio de Janeiro, IMPA.

[12] Coelho, F. U e Lourenço, M. L [2005] Um curso de álgebra linear. 2a ed. revista e ampliada. São Paulo: Edusp.

[13] Garcia, A. e Lequain, Y. [2006] Elementos de álgebra. Projeto Euclides, $4^{\mathrm{a}}$ ed., Rio de Janeiro, IMPA. 
[14] Gatermann, K. [2000] Computer Algebra Methods for Equivariant Dynamical Systems. Lecture Notes in Mathematics 1728, Springer-Verlag, Berlin-Heidelberg-New York

[15] Golubitsky, M., Stewart, I. e Schaeffer, D. [1985] Singularities and Groups in Bifurcation Theory. Vol. II, Appl. Math. Sci. 69, Springer-Verlag, New York.

[16] Goodman, R. e Wallach, N. R. [2001] Representations and Invariants of the Classical Groups. Cambridge University Press, Cambridge.

[17] Lamb, J.S.W. [1992] Reversing symmetries in dynamical systems. Physica A 25, 925-937.

[18] Lamb, J. S. W. e Roberts, R. M. [1999] Reversible equivariant linear systems. J. Diff. Eq. 159, 239-279.

[19] Roberto, L. A. F. [2004] Bifurcação de pontos de equilíbrio em sistemas acoplados com simetria do tipo produto coroa. Dissertação de mestrado, ICMC-USP.

[20] Sattinger, D. H. [1979] Group Theoretic Methods in bifurcation Theory. Lecture Notes in Mathematics 762, Springer, Berlin, New York.

[21] Singular - versão 3-0-4 [2008](http://www.singular.uni-kl.de/)

[22] Sturmfels, B. [1993] Algorithms in Invariant Theory. Texts and Monographs in Symbolic Computation, Springer-Verlag, Austria. 


\section{Índice}

ação, 7

de $\mathbf{S}_{n}$ em $V^{\otimes n}, 36$

de $\mathbf{S O}(2)$ em $\mathbb{R}^{2}, 8$

de $\Gamma$ ? $\mathbf{S}_{N}$ em $V^{N}, 58$

de $\Gamma^{N}$ em $V^{N}, 58$

de $\mathbf{O}(2)$ em $\mathbb{R}^{2}, 8$

de $\mathbf{S}^{1}$ em $\mathbb{C}, 8$

de $\mathbf{S}_{N}$ em $V^{N}, 58$

dual, 34

irredutível, 10

padrão, 8

trivial, 8

ação dual induzida

de $\Gamma$ ? $\mathbf{S}_{N}$ em $V^{N}, 63$

de $\Gamma^{N}$ em $V^{N}, 63$

anel

polinomial, 18

anel polinomial, 17

aplicação multilinear, 37

base de Hilbert, 17

caracter, 35

componente

conexa, 7

isotípica, 12

decomposição isotípica, 12, 14

espaços isomorfos, 9

fórmula

do traço, 29

fórmulas de Molien, 39

germe, 14

de aplicação

$\Gamma$ 乙 $\mathbf{S}_{N}$-reversível-equivariante, 63

T-equivariante, 18

-reversível-equivariante, 34
$\Gamma^{N}$-reversível-equivariante, 63

de função

$\Gamma$-anti-invariante, 34

$\Gamma$-invariante, 15

grupo

$\mathbf{G L}(n), 5$

cíclico $\mathbf{Z}_{n}, 6$

das permutações $\mathbf{S}_{n}, 79$

de Lie, 5

diedral $\mathbf{D}_{n}, 6$

especial ortogonal $\mathbf{S O}(n), 6$

ortogonal $\mathbf{O}(n), 6$

produto coroa $\Gamma$ ? $\mathbf{S}_{n}, 58$

Toro $\mathbf{T}^{n}, 6$

integral de Haar, 9

normalizada, 9

módulo livre, 23

operadores de Reynolds, 43

polinômio

$\Gamma$-anti-invariante, 34

$\Gamma$-invariante, 15

potência tensorial simétrica, 37

produto interno

invariante, 10

ortogonal, 10

produto tensorial, 36

propriedade universal, 36

relação, 17

representação, 7

$\sigma$-auto-dual, 35

$\sigma$-dual, 34

absolutamente irredutível, 18

irredutível, 10

representações isomorfas, 9

série de Hilbert, 38, 39 
subespaço

$\Gamma$-invariante, 10

de ponto-fixo, 25

irredutível, 10, 11

subgrupo

de isotropia, 25

conjugado, 25

\section{teorema}

de Fubini, 41

de Molien, 38

de Hilbert-Weyl, 17

de Molien (equivariante), 38

de Schwarz, 17 